\title{
Extended Research on
}

Detection of Deception

Using

\section{Volatile Organic Compound \\ (VOC) Emissions}

FINAL REPORT

Center for Human Reliability Studies

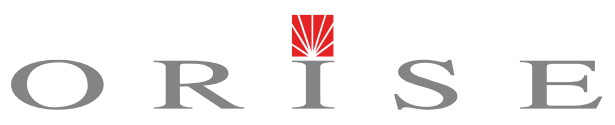


The Center for Human Reliability Studies (CHRS) provides a vehicle for achieving quality-oriented program support for DOE through research and analysis, technical guidance, and operational support in all areas of human reliability relating to occupational medicine, facility safety, personnel security, and the DOE Human Reliability Program. CHRS also conducts research on issues involving personnel reliability.

The Oak Ridge Institute for Science and Education (ORISE) is a U.S. Department of Energy institute focusing on scientific initiatives to research health risks from occupational hazards, assess environmental cleanup, respond to radiation medical emergencies, support national security and emergency preparedness, and educate the next generation of scientists. ORISE is managed by Oak Ridge Associated Universities.

This document was prepared by the Oak Ridge Institute for Science and Education (ORISE) through an interagency agreement with the U.S. Department of Energy (DOE). ORISE is managed by Oak Ridge Associated Universities under DOE contract number DE-AC05-06OR23100. 


\section{Extended Research on Detection of Deception Using Volatile Organic Compound (VOC) Emissions}

Final Report

Center for Human Reliability Studies

Oak Ridge Institute for Science and Education

Oak Ridge, Tennessee

ORISE 06-1690

December 2006 



\section{Table of Contents}

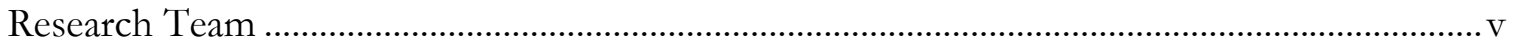

Executive Summary ……………………..................................................................................... vii

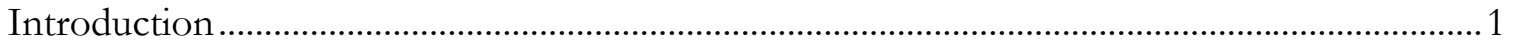

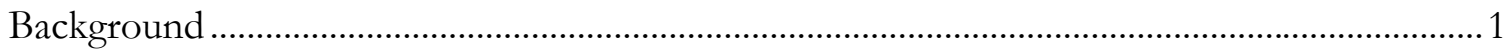

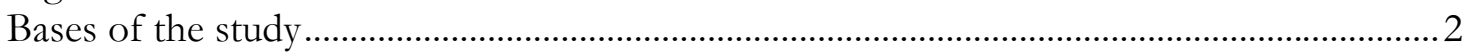

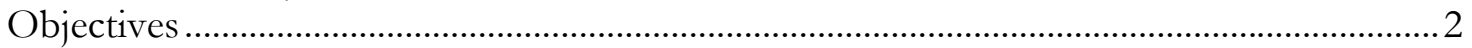

Project planning and schedule

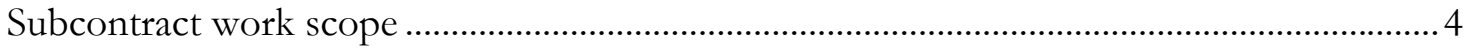

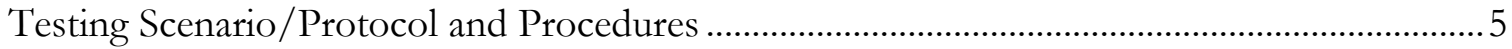

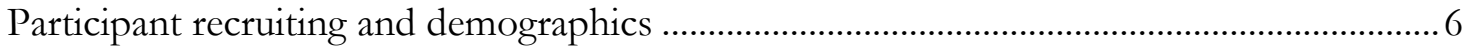

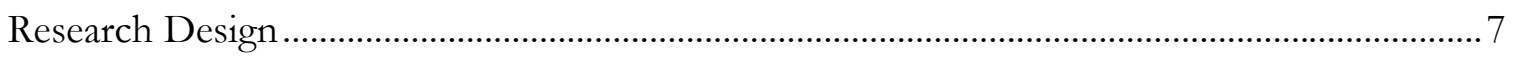

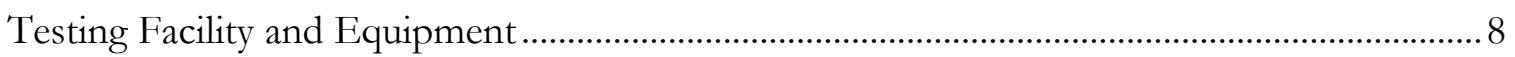

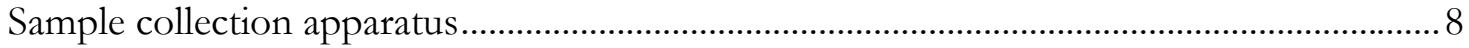

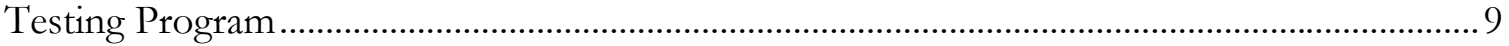

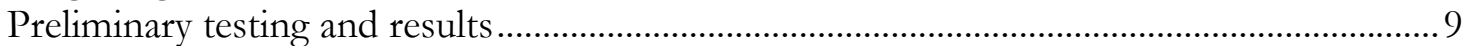

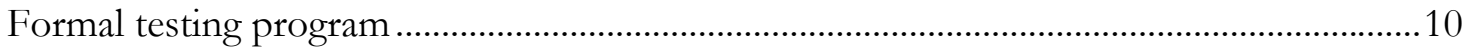

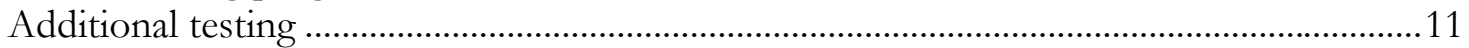

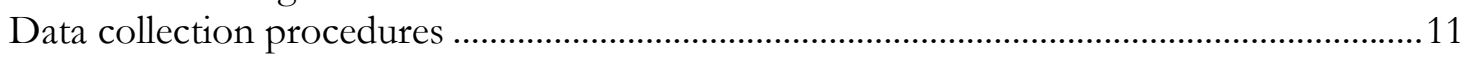

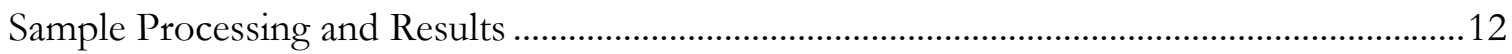

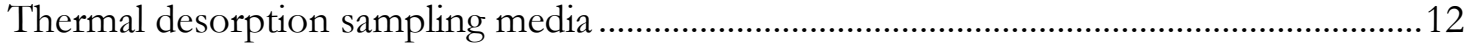

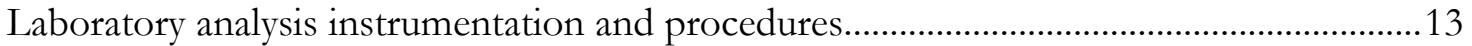

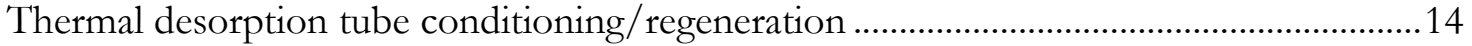

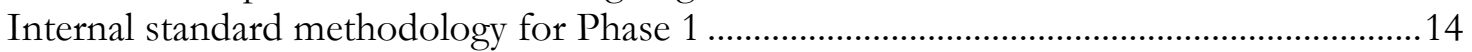

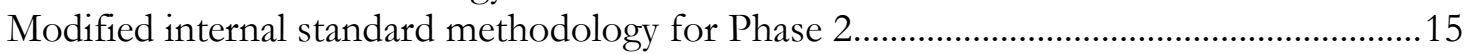

Representative analytical laboratory results .......................................................................... 17

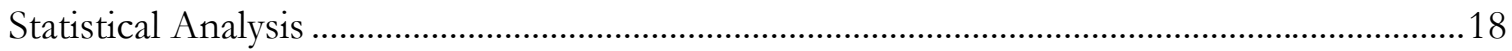

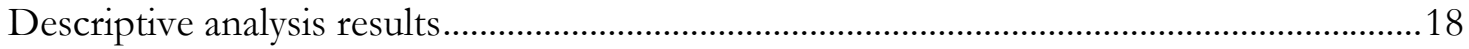

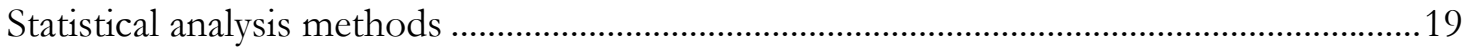

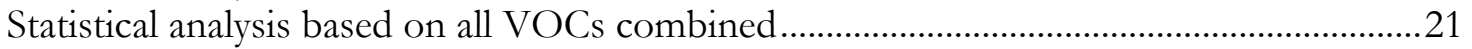

Statistical analysis based on groups of chemically similar VOCs ............................................24

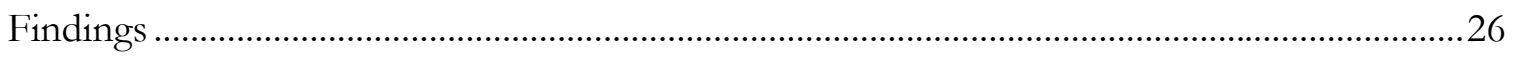

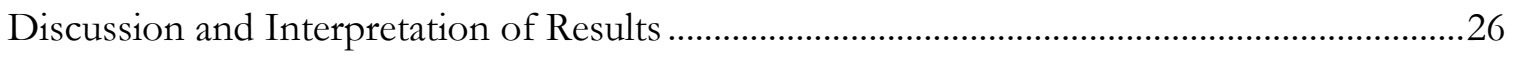

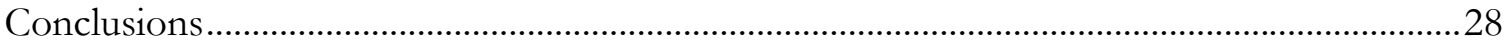

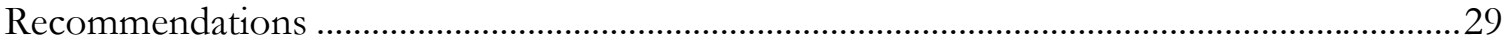

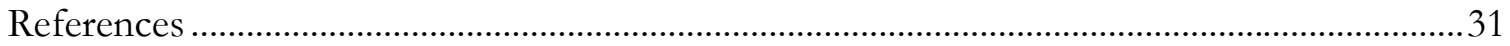




\section{Appendices}

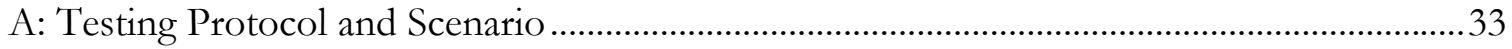

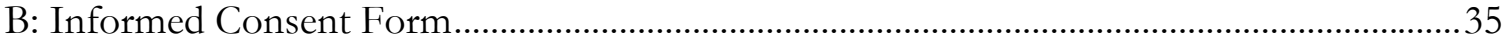

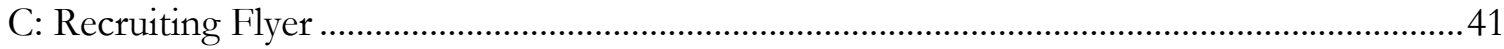

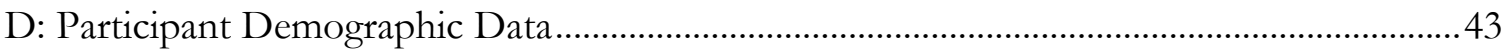

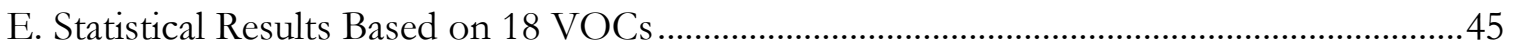

F. Statistical Results Based on Groups of Chemically Related VOCs .......................................51 


\title{
Research Team
}

\section{Center for Human Reliability Studies (CHRS)}

Don Watkins, Ph.D.

Jerry Eisele, Ph.D.

Janice Watkins, M.S.

Ed Frome, Ph.D.

Carolyn Alvarez, B.A.

Sandy Womble

Louise Wyatt

\author{
Y-12 Analytical Chemistry Organization \\ Gerald DeVault, Ph.D. \\ Darrin Mann, Ph.D. \\ Tom Adams, B.S. \\ John Hoffarth \\ Karen Hoffarth \\ Teri Smith
}




\section{Executive Summary}

This study follows initial work completed by the Center for Human Reliability Studies (CHRS) in 2005.

The current study was based on the following three premises:

- Human beings emit from skin surfaces natural volatile organic compounds (VOCs) that change in response to involuntary stresses induced by intentional deception (lying).

- These emissions are sufficient in quantity to be detected and measured reliably by standard analytical instrumentation.

- These responses can be analyzed to indicate heightened stress states associated with deception.

The principal objectives of this study were to:

- Develop a strong and realistic testing scenario to elicit consistent emotional responses such as those found in the initial CHRS research.

- Develop an expanded and comprehensive list of target VOCs to effectively relate skin emissions to stresses induced by intentional deception.

- Develop methods of statistical analysis to base deception detection results on data from multiple VOCs simultaneously.

- Investigate and evaluate applying emerging analytical methods, such as solid-phase micro-extraction, to detect and quantify VOCs faster and more reliably than present instrumentation.

- Consider feasibility and requirements for developing a real-time, quick-response VOC detection and analysis system based on emerging micro-cantilever technology.

- Continue the effort to detect and quantify heavier molecular weight semi-volatile organic compounds (SVOCs) to aid in detecting deception. 
The major finding in this study was that, in general, participant responses to stress induced by deceptive behavior registered an increase in VOC emission levels compared to neutral conditions. Results from an earlier study conducted by CHRS using emissions of VOCs to detect strong emotional response in individuals (Watkins et al., 2005) showed markedly decreased emission levels in several VOCs in response to subjects viewing a video of an intensely fearful situation. The results of the two CHRS studies are consistent with another reported study in which the use of infrared thermography showed facial skin surface temperature increased in response to induced emotions of sadness, joy, and anger, but found that temperatures decreased when these same subjects experienced fear (Tanaka and Ide, 1998).

It was concluded that, when responses are not intense, it is necessary to focus on groups of chemically similar VOCs rather than individual chemicals to more readily indicate deception. Also, using groups of similar chemicals rather than all VOCs combined provides increased sensitivity and resolution in mass spectrometry results to indicate deception.

The work reported here represents advancement toward ultimately developing a workable and reliable system that can serve as a viable adjunct to the polygraph or function effectively as a lightweight, portable deception detection device for field use. Viable statistical methods were developed and analysis functions written to produce both graphic and numeric results. Details are presented throughout the Discussion and Interpretation of Results section of this report (see page 26).

The major conclusion/recommendation drawn from this study was that research should be continued to assess the feasibility of adapting cutting-edge VOC sensor technologies to enhance detector capability toward the goal of achieving shortened, real-time response to changing VOC concentrations as a function of induced stress levels in humans. 


\section{Introduction}

Detailed discussion of the limitations of traditional polygraph testing is found in the final report on the initial research on using volatile organic compound (VOC) emissions to detect intentional deception (Watkins et al., 2005). In brief, the extensive evaluation of polygraph effectiveness performed by the National Research Council (2003) highlights the lack of research to develop either viable alternatives to the polygraph or technologies that can work in conjunction with the polygraph to augment its capabilities. This research, sponsored by the National Security Agency, represents a continuing effort to advance technology in this area. ${ }^{*}$

A system that captures and analyzes VOC emissions from skin surfaces may offer a viable alternative method to the polygraph instrument currently in use for detecting deception in U.S. government settings. Like the involuntary autonomic central nervous system response data gathered during polygraph testing, VOC emissions from the skin may provide data that can be used to detect stress caused by deception. Detecting VOCs, then, may present a noninvasive, non-intrusive method for observing, recording, and quantifying evidence of stress or emotional change.

\section{Background}

There is a definite lack of published research on investigations specifically centered on the relationship between human emotional stress and changes in the emission of natural VOCs and heavier molecular weight, semi-volatile organic compounds (SVOCs) from skin surfaces. However, research in related areas, including commercial product development, has identified many specific types of natural VOCs emitted from skin surfaces. For example, in one study, over 135 such VOCs, primarily ketones and alcohols as well as alkanes and alkenes, were identified (Ellin et al., 1974). In another study, 346 VOC peaks were detected

\footnotetext{
* It is acknowledged that a major underlying problem in all research in methods to detect deception is that there is no absolute certainty that the physical stress responses registered by study participants would be similar to those who are actually in jeopardy of having to face the consequences of being discovered lying.
} 
and identified, the most prominent being organic acids, carboxylic acids, sulfides, and aldehydes (Bernier et al., 2000).

Two other studies worthy of note relate to investigations pertaining to identifying chemical marker compounds found in fingerprints. The first study (Asano et al., 2002) concentrated on investigating changes in heavier molecular weight SVOCs typically present in skin oils found in the palm of the hand and in fingerprints. Results of that study identified the presence of fatty acids, esters, cholesterol, and squalene in fingerprint residues. The other research (Zhang et al., 2005) focused on 10 components out of the 35 initial VOCs identified that included alkanes, an ether, and alcohols. Results of these studies were considered in focusing attention on particular VOCs in the present CHRS research.

\section{Bases of the study}

This study is based on the following three premises:

1. Human beings emit from skin surfaces natural VOCs that change in response to involuntary stresses induced by deceptive behavior.

2. These emissions are sufficient in quantity to be detected and measured reliably by standard analytical instrumentation.

3. These responses can be analyzed to indicate heightened stress states associated with deception.

\section{Objectives}

The purpose of the research was to build on and extend the body of knowledge developed in the initial study, "Detection of Deception Using Volatile Organic Compound (VOC) Emissions" (Watkins et al., 2005).

The specific objectives of this study were to:

- Develop a strong and realistic testing scenario to elicit deceptive responses to a realworld simulation to confirm the results obtained in the first study.

- Develop an expanded and comprehensive target list of VOCs to detect and quantify for the purpose of relating skin emissions to stress induced by intentional deception, 
initially focusing on the six most prominent VOCs identified previously and increasing the number of candidate VOCs.

- Develop and employ a quick-response, flow-indexing sampling system to stabilize timing of sample acquisition and speed collection of data.

- Determine the most advantageous experimental design and select appropriate statistical analytic approaches to match the design.

- Develop statistical methods to analyze VOC data sets.

- Explore the possibilities of expanding the scope of the research to include detection and quantification of SVOCs.

- Investigate and evaluate applying emerging analytical methods, such as solid-phase micro-extraction (SPME), to detect and quantify VOCs (and possibly SVOCs) faster and more reliably.

- Address feasibility and requirements for developing a real-time, quick-response VOC (and possibly SVOC) detection and analysis system based on micro-cantilever technology.

Investigators evaluated expanding the scope of the research to include using SPME analytical methods to identify SVOCs and VOCs. After initial experimentation in the analytical laboratory to assess feasibility of detecting VOCs and SVOCs reliably, it was apparent that the SPME analytical method would neither yield practical results in detecting and measuring SVOCs nor present a clear advantage over the thermal desorption tube and gas chromatography/mass spectrometry (GC/MS) methods used to date.

Basing a real-time deception detection system on micro-cantilever technology to detect and quantify VOCs was also evaluated. Potential advantages included faster response time and inclusion of a broader spectrum of VOCs. However, investigation revealed that at its present stage of development this technology would not meet projected operating requirements because of low sensitivity of the primary sensor arrays. It was concluded that applying the SPME method for SVOCs and further evaluating micro-cantilever technology for a real-time 
system were not feasible within the timeframe and resources of this project. Therefore, data on SVOCs were not collected during the study.

\section{Project planning and schedule}

During statistical analysis of initial results (see page 18), irreconcilable inconsistencies, which compromised the reliable interpretation of the data, appeared in some of the results. To address the problem, a three-month nocost extension was proposed and accepted. This extension, to December 29, 2006, allowed acquisition of additional data using multiple internal chemical standards instead of just one (to be discussed). This was accomplished, with the project completion date moving from September 29, 2006 to December 29, 2006.

Table 1. Project plan elements and schedule

\begin{tabular}{|l|l|}
\hline \multicolumn{1}{|c|}{ Element } & \multicolumn{1}{c|}{$\begin{array}{c}\text { Completion } \\
\text { date 2006 }\end{array}$} \\
\hline Plan, schedule, and allocate resources & September 15 \\
\hline Evaluate required analytical modifications & September 26 \\
\hline Develop improved testing scenario and procedures & September 26 \\
\hline $\begin{array}{l}\text { Obtain ORSIRB* approval for protocol } \\
\text { modification }\end{array}$ & September 26 \\
\hline Recruit 16 additional participants & October 10 \\
\hline Implement analytical method modifications & October 10 \\
\hline Conduct Phase 2 testing (w/Y-12 ACO) & October 17 \\
\hline Test sample analysis (80 samples at Y-12 ACO) & October 26 \\
\hline Analyze and interpret data & November 29 \\
\hline Prepare draft report & December 8 \\
\hline Review and edit report & December 15 \\
\hline Deliver final report & December 29 \\
\hline
\end{tabular}

*Oak Ridge Site-wide Institutional Review Board

Project plan elements including Phase 2 participant testing and the revised completion schedule for the time extension appear in Table 1.

\section{Subcontract work scope}

The Y-12 Analytical Chemistry Organization (ACO) was subcontracted to provide analytical capability and specialized equipment for the project. ACO is part of the National Nuclear Security Administration (NNSA) Y-12 National Security Complex in Oak Ridge, Tennessee. 
Scope of the Y-12 ACO work included the following elements:

1. Assist in identifying the expanded target list of most likely VOCs (and SVOCs) for study; reevaluate the six most prominent VOCs from the earlier study and expand by at most 16

2. Design and fabricate the flow-indexing VOC sample acquisition system

3. Perform preliminary laboratory testing of the new sample acquisition system; evaluate operation of the new system and calibrate analytical laboratory equipment

4. Determine the most effective single and multiple internal standard compounds

5. Assist in the formal testing program with human subjects

6. Analyze acquired samples using the thermal desorption method with GC/MS; interpret spectral data

7. Prepare the sample analysis report and provide data in a format suitable for statistical analysis

A consulting subcontract was also placed for specialized statistical expertise.

\section{Testing Scenario/Protocol and Procedures}

The basic deception scenario and initial protocol were developed specifically for this study. As testing proceeded minor modifications were made to the protocol to improve the effectiveness of the scenario. The basic scenario, however, remained the same throughout the testing program. The scenario consisted of the participants lying about having a piece of criminal evidence in their possession at the time of questioning. Testing proceeded in four phases: 1a, 1b, 1c, and 2. Minor modifications were made to the protocol to improve the effectiveness of the scenario. Between the phases, CHRS investigators recruited participants, obtained consent, and collected and analyzed the test data.

The testing protocol and scenario are presented in Table 2 and Appendix A. The Phase 2 version is presented in Appendix A because it represented the most advanced version of the four protocols and scenarios used in this study. Only small changes took place in the protocol advancing to Phase 2; therefore the others are not presented. Table 2 shows the 
differences between the four testing phases. (Other differences between phases are discussed in the Testing Program section.) The structure of the specific questions asked during data collection was identical between phases because only one deception item was involved in each case. Questioning involved asking two relevant questions specific to the item of interest and two irrelevant or neutral questions (refer to Appendix A). "Random selection" of the item in Table 2 refers to the participant choosing, in a random process, between actually having the item and having no knowledge of the item.

All consent forms and changes in testing protocol were formally reviewed and approved by the Oak Ridge Site-wide Table 2. Testing profiles

\begin{tabular}{|c|c|c|}
\hline \multicolumn{3}{|c|}{ Table 2. Testing profiles } \\
\hline Phase & $\begin{array}{c}\text { Deception } \\
\text { item }\end{array}$ & $\begin{array}{c}\text { Random } \\
\text { selection }\end{array}$ \\
\hline 1a & Cocaine & Yes \\
\hline 1b & Cocaine & Yes \\
\hline 1c & Cocaine & Yes \\
\hline 2 & Handgun & No \\
\hline
\end{tabular}
Institutional Review Board (ORSIRB) before use to ensure full compliance with applicable federal requirements. A representative consent form used in this study appears in Appendix B. Only one consent form is included because of the similarities between the versions. During the interview conducted before study participation, each subject carefully reviewed the consent form; any questions were answered before it was signed. In addition, after acceptance of participation and before testing, each subject was provided an overview of sampling system operation, data collection requirements, and the testing scenario.

Following testing and data collection, each subject was debriefed and given compensation for participating in the study. As part of the debriefing, the participant was informed that the testing scenario was a simulation and that the criminal evidence was a prop. During the debriefing, the participant was asked about the level of stress experienced during the scenario. In addition, each participant was cautioned not to discuss the testing scenario with anyone to preclude preconditioning or biasing of the responses from subsequent participants.

\section{Participant recruiting and demographics}

A total of 48 participants for the testing program were recruited from Oak Ridge educational institutions, medical facilities, local government offices, and other high-traffic areas. The flyer shown in Appendix $\mathrm{C}$ was used to facilitate recruiting. A demographic breakdown is 
presented in Appendix D. Of the participants recruited, 45 were white; two were Hispanics; and one was black. Although the ethnic distribution of the participants did not represent a cross-section of the general U.S. population, gender and age were more evenly distributed.

\section{Research Design}

The research design provided the structure for obtaining data required to assess the feasibility of using VOC (and SVOC) emission levels to indicate physiological changes induced by stress. These changes could then be analyzed to detect deceptive behavior and establish the parameters within which to potentially develop a practical working system.

The research design centered on the following protocol:

- Divide the testing program into separate phases corresponding to distinct benchmarks in the evolution of increasingly stronger deception scenarios

- Use control participants_-individuals who did not possess the item of evidence-in early phases of the testing program to provide information on the potential incidence of false positive results

- Use only one item for participants to lie about possessing to increase the positive indication of deception

- Use two relevant and two irrelevant questions to collect data, keeping the volume of data manageable for detailed statistical analysis

- Reduce the time interval for data collection initially to one minute to test the feasibility of obtaining meaningful data in a shortened time consistent with anticipated field application of this technology

- Obtain and analyze periodic environmental samples from the testing room to confirm that no ambient VOCs appear during data collection

Control participants were provided a modified consent form that did not identify the item of evidence. In all other respects, they proceeded through the identical testing process with the same questions as those who possessed the deception item. 


\section{Testing Facility and Equipment}

The facility used for the testing program was located in an office building in Oak Ridge on the Oak Ridge Institute for Science and Education South Campus. The test facility consisted of a climate-controlled testing room, an evidence room containing the deception item under guard of a uniformed law enforcement officer, and an interview room for participants to be introduced to the study, review and sign the consent form, and be debriefed.

Test equipment was provided by Y-12 ACO. This included the flow-indexing sampling apparatus, plastic tubing flow lines, flow regulating meter, and sampling air pump. Testing operations were directed by a CHRS researcher. Y-12 provided the equipment operator(s) and secured the samples.

\section{Sample collection apparatus}

The sample collection system used in Phases 1 and 2 is shown in Figures 1 and 2. A 6-way selection valve (also called the flow-indexing valve), made from PEEK ${ }^{\mathrm{TM}}$ (polyetheretherketone), was purchased from Upchurch Scientific, Inc. The flow-indexing valve was used to selectively divert the sampled VOCs between individual thermal desorption tubes. Five thermal desorption tubes were used per participant (baseline sample plus four samples, one for each question). Flexible Teflon $\AA$ tubing (1/4" outer diameter) connected the individual ports on the flow-indexing valve to the individual thermal desorption tubes and a 6-port collection manifold (also purchased from Upchurch). The thermal desorption tubes were connected to the Teflon tubing by Teflon Swagelok

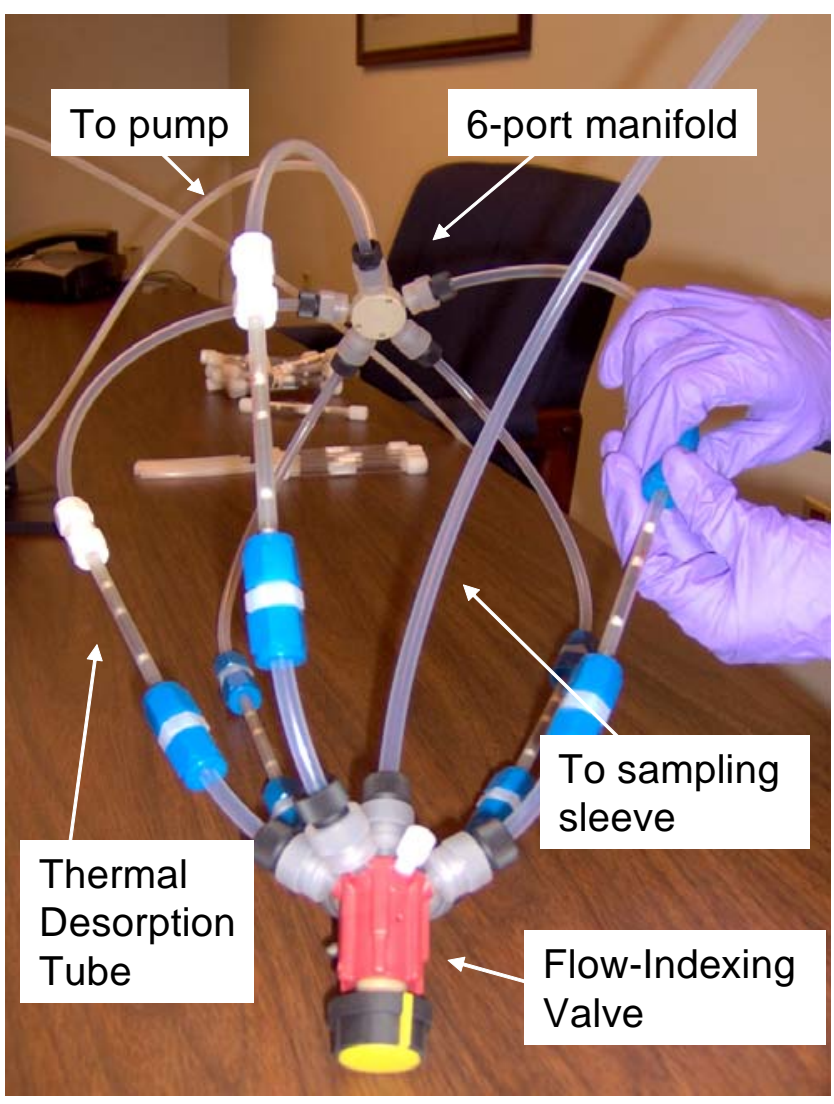

Figure 1. Flow-indexing system 


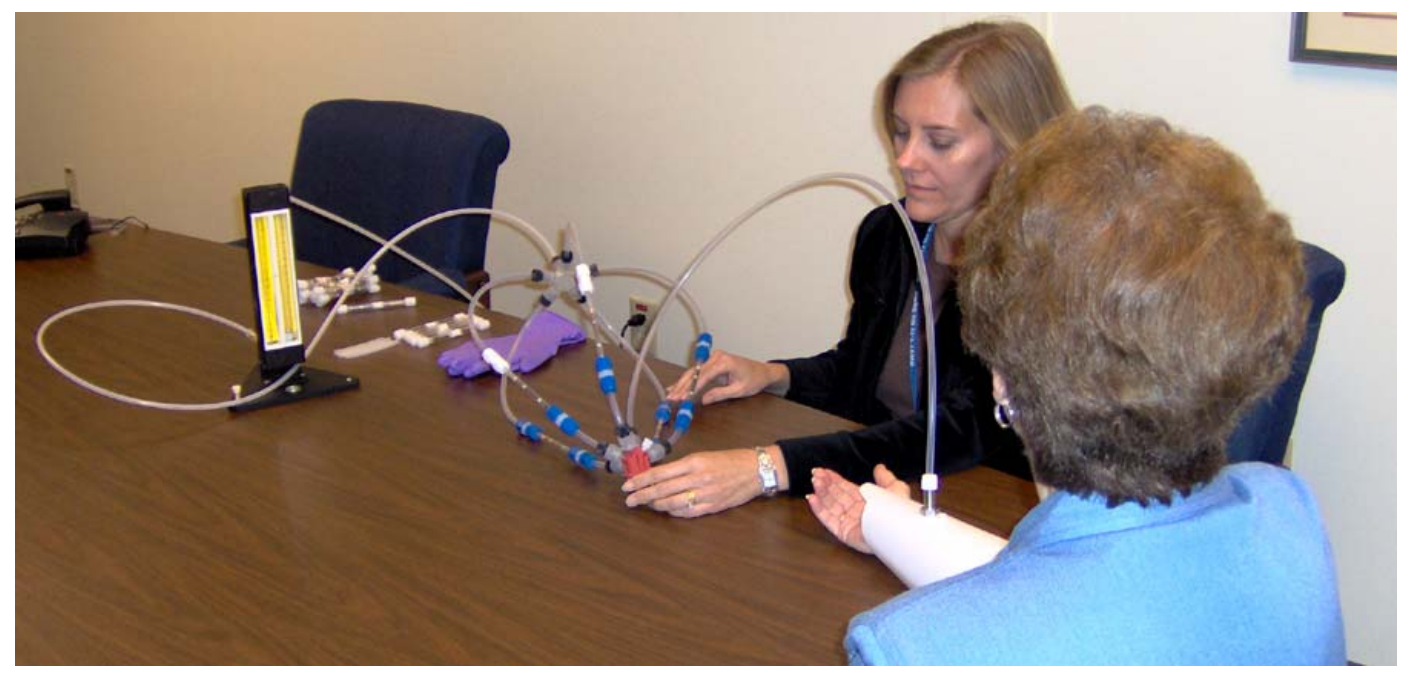

Figure 2. Test setup with Teflon sampling sleeve

union fittings (see Figure 1). The outlet of the manifold was connected to a vacuum diaphragm pump that maintained $\sim 800$ milliliters per minute $(\mathrm{mL} / \mathrm{min})$ flow rate through the system and was regulated by means of a flow meter (Figure 2). The inlet of the 6-way selection valve was connected to the Teflon sampling sleeve (Figure 2) used for sampling VOCs from each participant's forearm. All materials used to construct the sampling system were known to be inert and not interact with the VOCs of interest.

\section{Testing Program}

\section{Preliminary testing and results}

Preliminary testing was performed to evaluate the stronger and more realistic deception scenario developed for this study and to test the operability of the VOC sample collection system. The new system included (1) the flow-indexing valve and tubing apparatus that allows the sequential acquisition of five samples in rapid succession (Figure 1); and (2) the rigid Teflon sleeve with the sampling port mounted on the side that reduces the headspace volume, thereby minimizing the dilution of VOC samples obtained adjacent to the skin (Figure 2). The sampling pump flow throughout the testing program was maintained at a steady rate of 800 to $820 \mathrm{~mL} / \mathrm{min}$. The required pump rate was increased from the previous study to deposit more VOCs on the sampling tube sorption matrix. 
In addition, preliminary tests were conducted at Y-12 to determine the feasibility of using SPME methods for VOC analysis, and possibly for SVOC analysis, but those methods proved ineffective for this study. Evaluation of available micro-cantilever VOC sensing instrumentation and equipment revealed that currently achievable sensitivity thresholds would be insufficient for application to a workable real-time system utilizing VOC detection technology.

\section{Formal testing program}

The subjects were asked to participate in a scenario that evolved during the process described in Table 3, which expands the data in Table 2 and provides additional information on the parameters of Phases 1a, 1b, 1c, and 2.

\begin{tabular}{|c|c|c|c|c|c|c|c|}
\hline \multicolumn{7}{|c|}{ Table 3. Testing program parameters } \\
\hline Phase & Test dates 2006 & $\begin{array}{c}\text { Number } \\
\text { of } \\
\text { subjects }\end{array}$ & $\begin{array}{c}\text { Deception } \\
\text { item }\end{array}$ & $\begin{array}{c}\text { Random } \\
\text { selection }\end{array}$ & $\begin{array}{c}\text { Number } \\
\text { of control } \\
\text { subjects }\end{array}$ & $\begin{array}{c}\text { Sampling } \\
\text { interval }\end{array}$ & $\begin{array}{c}\text { Guard in } \\
\text { testing } \\
\text { area }\end{array}$ \\
\hline 1a & May 8, 9 & 8 & Cocaine & Yes & 4 & $\begin{array}{c}1.0 \text { min }+ \\
0.5 \text { min } \\
\text { sweep }\end{array}$ & No \\
\hline 1b & May 15, 16 & 8 & Cocaine & Yes & 0 & $\begin{array}{c}2.0 \text { min } \\
\text { (no } \\
\text { sweep) }\end{array}$ & No \\
\hline 1c & May 22, 23, 24 & $15 *$ & Cocaine & Yes & 3 & $\begin{array}{c}2.0 \text { min } \\
\text { (no } \\
\text { sweep) }\end{array}$ & Yes \\
\hline 2 & Oct. 11, 12,16,19 & 16 & Handgun & No & 0 & $\begin{array}{c}2.5 \text { min } \\
\text { (no } \\
\text { sweep) }\end{array}$ & Yes \\
\hline
\end{tabular}

* On May 24, one participant did not arrive.

The total number of participants scheduled for testing in Phases 1a, 1b, 1c, and 2 was 48; however, on May 24 one did not arrive, resulting in 47 participants providing data. In Phase 2, one participant did not follow the protocol and the data was eliminated from the analyses.

Five samples were obtained from each participant during data collection. The first sample was a baseline sample for the individual participant, with four subsequent samples taken as participants answered questions relating to the testing scenario. Data collection time for each sample varied according to phase (see Table 3). 


\section{Additional testing}

Analysis and preliminary evaluation of test data from Phases 1a, 1b, and 1c suggested systematic inconsistencies in results, which are discussed fully in the Statistical Analysis section. These results may have been caused by correlations within subjects in the data values of the internal standard used, fluorobenzene, which was employed to normalize response data. ${ }^{*}$ To rectify this situation, the research sponsor granted a no-cost schedule extension to conduct additional testing (Phase 2). The solution included using multiple standards at appropriate times in the analytical laboratory process (discussed under the section on modified internal standard methodology for Phase 2).

\section{Data collection procedures}

The following sampling and data collection procedures were used throughout testing. Refer to Table 3 for specific operating parameters.

1. Take one environmental background (equipment blank) data sample through the test apparatus at the beginning of each testing session with the person's forearm not in the sampling sleeve.

2. Obtain a baseline data sample specific to that individual with the participant's arm placed in the Teflon sampling sleeve and with no question being asked. (Sampling time duration varied between individual phases, see Table 3.)

3. Obtain a data sample with the subject's arm held in the sampling sleeve while he or she is responding to each neutral question.

4. Obtain a data sample immediately after each relevant, directed-lie (deception) question is asked with the person's arm held in the sampling sleeve.

5. In each step, data are collected for the prescribed time interval starting immediately when the question is asked.

\footnotetext{
* Normalizing is the accepted practice of adjusting data values by dividing each data value by its corresponding fluorobenzene value. Spiking the thermal desorption tubes with an internal standard compound helps to compensate for the imprecision inherent in the sampling procedures and the analytical methods. An internal standard is used in this way to adjust data values for sample variation and instrument variation between test subjects.
} 
The researchers used the following procedures during questioning of the subject:

1. With the sampling pump running, the researcher asked the question.

2. The participant was asked, after hearing the question, to wait 5 to 10 seconds before answering to provide time to think about the question and the answer and to allow the time to experience the stress of a reaction more fully. (Sampling duration varied between individual phases; see Table 3.)

3. After the subject answered the question, the researcher waited 5 to 10 seconds and asked the question again, perhaps worded slightly differently.

4. The participant was asked, after hearing the question the second time, to again wait 5 to 10 seconds before answering to provide time to think about the question and the answer and to allow the time to experience the stress reaction more fully.

5. No question was asked more than twice.

\section{Sample Processing and Results}

\section{Thermal desorption sampling media}

Thermal desorption tubes trap and retain a wide variety of organic compounds for qualitative and quantitative analyses. These tubes contain special adsorbent materials that facilitate retention and thermal desorption of compounds of interest, while remaining thermally stable. For this study, the tubes selected were Carbotrap 300 desorption tubes manufactured by Supelco. These tubes contain three different sorption beds: Carbotrap B, Carbotrap C, and Carboseive S-III. Carbotrap B and Carbotrap C beds are useful for trapping airborne organic compounds containing four to twelve carbon atoms. These materials are manufactured at high temperatures to reduce bleeding into the GC/MS system. They are hydrophobic to minimize the displacement of compounds by water. Carboseive S-III is a high surface area carbon molecular sieve that is useful for trapping smaller organic compounds. Although it retains slightly more water than the other beds, its pure carbon framework permits efficient desorption of compounds without significant loss. 


\section{Laboratory analysis instrumentation and procedures}

The VOCs trapped on the sampling tube matrix were analyzed using a GC/MS instrument (Hewlett Packard gas chromatograph [HP5890] and mass spectrometer [HP5972]). A

Dynatherm Model 890 thermal-tube desorber was connected to the GC inlet. The apparatus is shown in figures 3 and 4 . The thermal desorption tube containing the trapped VOCs was placed in the sample inlet as shown in Figure 3. The VOCs were desorbed for $9 \mathrm{~min}$ at $310^{\circ} \mathrm{C}$. The transfer lines (between the sample inlet and the GC) were maintained at a temperature of $220^{\circ} \mathrm{C}$. The VOCs were desorbed onto the GC column [Restek RTX-Volatile (60

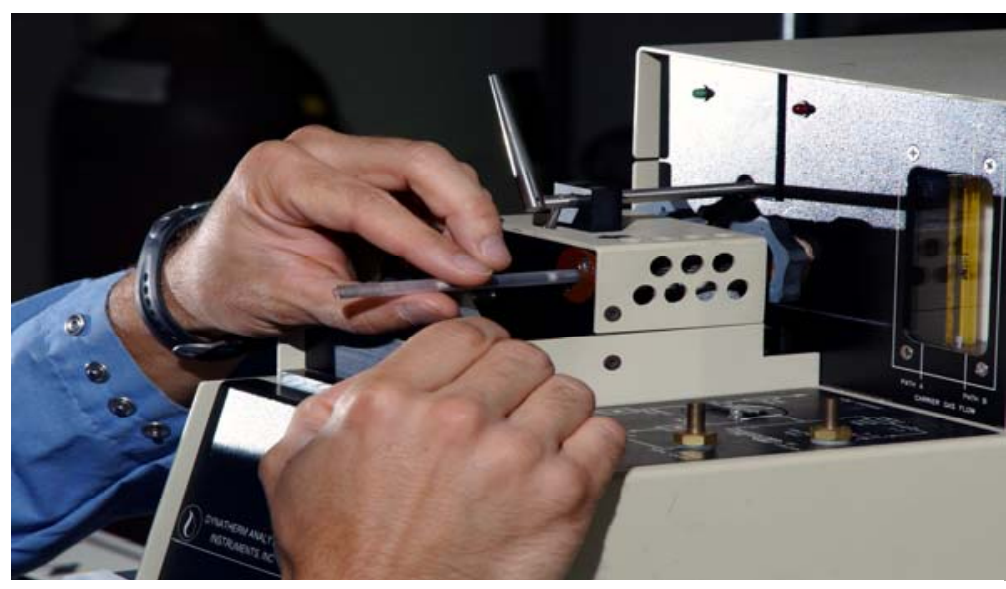

Figure 3. Thermal desorption tube injector $\mathrm{m}, 0.25 \mathrm{~mm}$ internal diameter, $1 \mu \mathrm{m}$ film thickness)]. The VOCs were separated based on their boiling point and their interaction with the stationary phase on the GC column. After separation, the VOCs were carried into a MS for detection. The GC operating parameters were $180^{\circ} \mathrm{C}$ injection temperature and $250^{\circ} \mathrm{C}$ detector inlet temperature. The GC oven temperature cycling program started with an initial temperature of

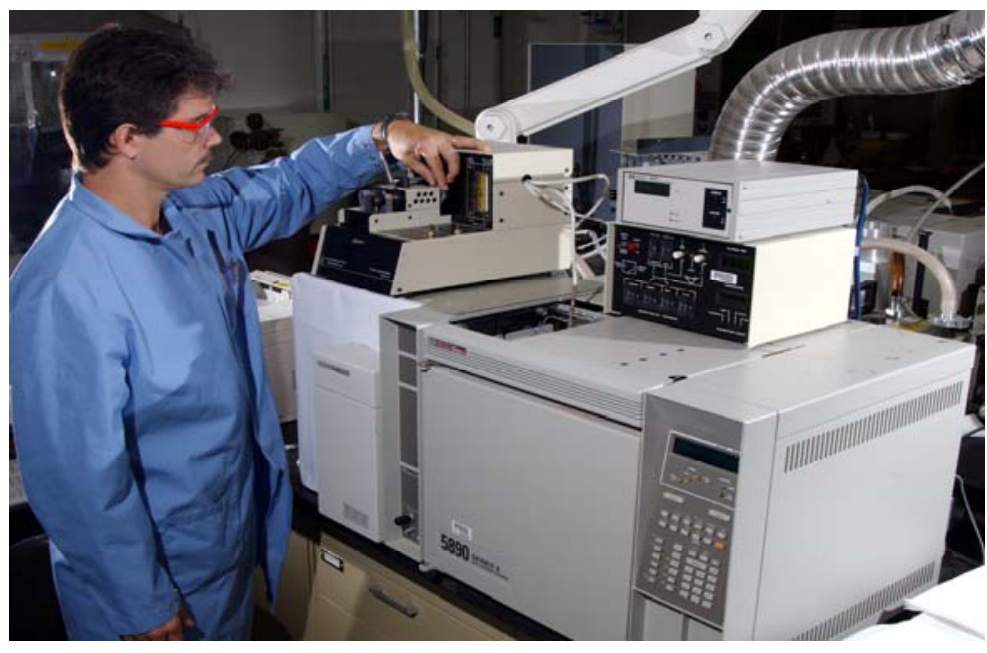

Figure 4. GC/MS instrument $37^{\circ} \mathrm{C}$ for $4 \mathrm{~min}$ and increased at a rate of $10^{\circ} \mathrm{C} / \mathrm{min}$ to the final temperature of $240^{\circ} \mathrm{C}$, which was held for $7 \mathrm{~min}$.

After collecting samples during testing, the thermal desorption sampling tubes were taken to the Y-12 analytical laboratory and prepared for analysis in the GC/MS. Figure 3 shows a 
typical sampling tube being inserted into the VOC desorption unit mounted atop the GC/MS by a technician.

In Figure 4, the technician is applying the inert carrier gas stream to sweep the desorbed VOCs into the GC/MS unit that is used to identify and quantify the specific VOC spectral peaks in the sample.

\section{Thermal desorption tube conditioning/regeneration}

Conditioning and regeneration of desorption tubes for multiple reuse was performed immediately after each analytical measurement. After each analysis, the desorption tube was heated again and a measurement taken on the effluent desorbed from the tube. This step served two functions: (1) ensuring that removal of the constituent adsorbed during the earlier operation was complete, and (2) ensuring that there was no VOC carryover into the next analysis. This step was followed by desorption tube reconditioning, as prescribed by the manufacturer. The process included reconditioning the tubes after heating to $350^{\circ} \mathrm{C}$ for one hour under helium flowing at $10 \mathrm{~mL} / \mathrm{min}$ to drive off any residual volatiles that could compromise subsequent data.

\section{Internal standard methodology for Phase 1}

Prior to use in Phases 1a, 1b, and 1c, each thermal desorption tube was spiked with a single internal standard, fluorobenzene. The purpose was to ensure consistency between sample analyses and to permit a basis for normalization of the data. The internal standard allows reduction of the natural variation inherent in the sampling and analytical instrument methods. Fluorobenzene was chosen as the internal standard because it is the recommended compound generally used in analytical procedures involving analysis for VOCs.

Fluorobenzene exhibits retention times similar to the VOCs of interest in this study, yet does not coelute or cause any chromatographic interference with the targeted compounds identified in the study.

Each sample tube was spiked with $100 \mathrm{ng}$ (nanogram) of fluorobenzene prior to the sampling event using the following procedure:

- The fluorobenzene stock solution was prepared at a concentration of $100 \mathrm{ng} / \mu \mathrm{L}$. 
- After connecting the sample tube to a side-port, a Hamilton gastight syringe was used to inject a small volume $(1.0 \mu \mathrm{L})$ of the stock solution into the injection port of the Dynatherm thermal desorption unit.

- The internal standard was then thermally transferred to the sample tube while maintaining a temperature of $230^{\circ} \mathrm{C}$ for 6 min while sweeping the injection port with helium at a flow rate of $10 \mathrm{~mL} / \mathrm{min}$.

\section{Modified internal standard methodology for Phase 2}

During the sample tube conditioning stage for Phase 2, the thermal desorption tubes were standardized by flash heating for $3 \mathrm{~min}$ with $100 \mathrm{ng}$ of two standards: fluorobenzene and cyclohexanone. Cyclohexanone was added to the sample tubes along with the fluorobenzene in Phase 2. Cyclohexanone was chosen because it has a polarity that is similar to the compounds of interest, it was not detected in any participant responses, and it does not coelute with other VOCs or produce spectral interference.

After obtaining sample data from the participants and prior to gas chromatography analysis, a third standard of $100 \mathrm{ng}$ of toluene-d8* was flash heated for 3 min onto the individual thermal desorption tubes. The toluene-d8 was added to monitor performance of the analytical system after samples were taken from the participants. This standard would only be affected by variations attributable to instrument drift.

During Phase 2 sample analyses, laboratory personnel noticed that the GC/MS might be malfunctioning. As a means of evaluating this effect, a statistical study was performed on the internal standard cyclohexanone data, which were divided by the corresponding fluorobenzene values. This analysis evaluated the internal standard response for the 15 subjects in Phase 2 to determine if a trend was present. Figure 5 shows normalized cyclohexanone internal standard data for the 15 subjects, with five analyses per subject.

\footnotetext{
* Toluene-d8 is fully deuterated toluene; that is, toluene in which all hydrogen atoms have been replaced by deuterium.
} 
Subject vs. percent recovery

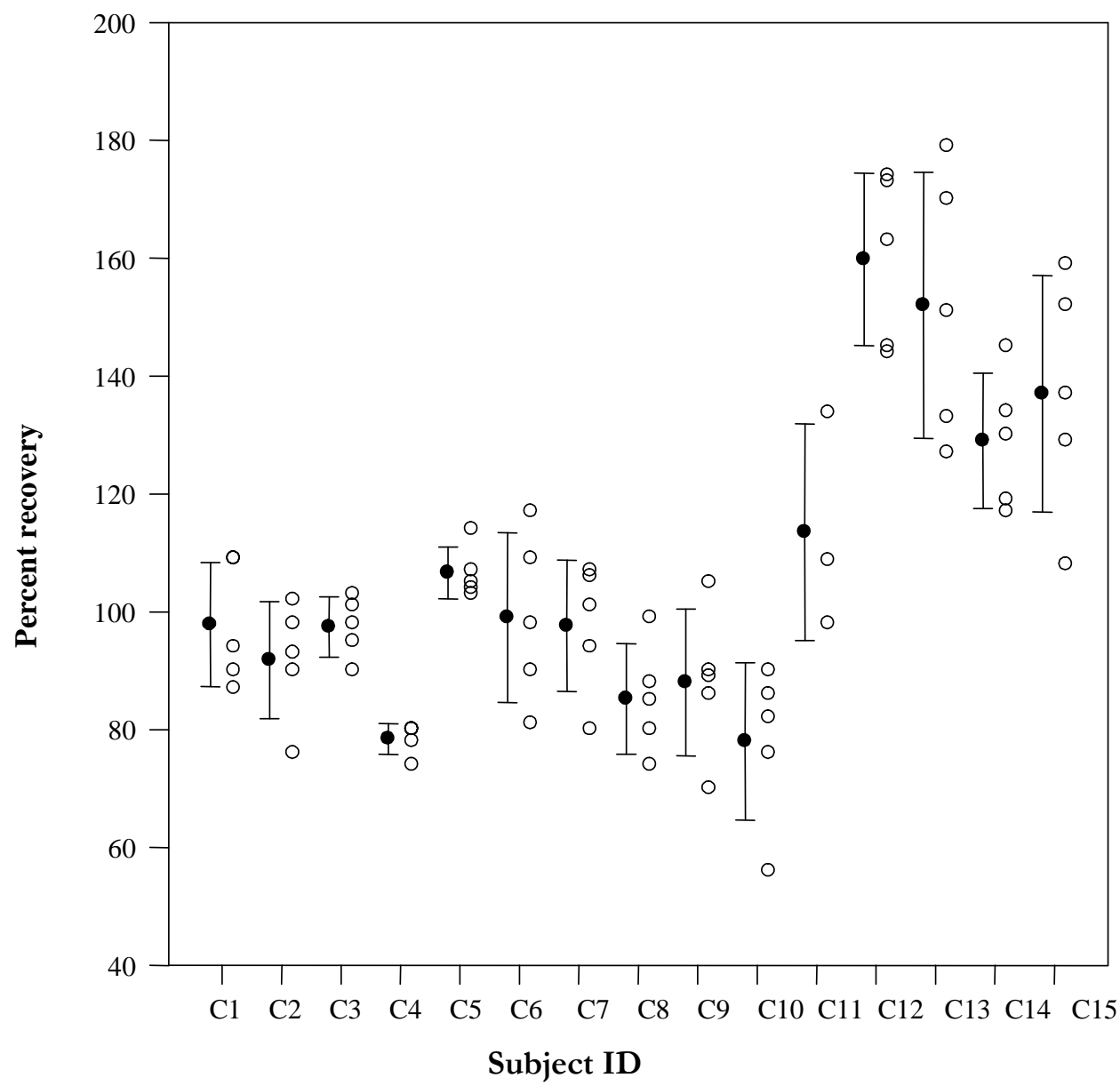

Figure 5. Cyclohexone point and column mean values

Two groups, C1 through C10 and C11 through C15 were visually identified. A statistical test, the Mann-Whitney Rank Sum Test, was performed to compare the two groups. As determined by the test, the difference in the median values between the two groups was greater than would be expected by chance; that is, there is a statistically significant difference. Most importantly, the shift in the internal standard response corresponded closely with the instrument problems that occurred during Phase 2 sample analyses. It was concluded that because the data were suspect beyond C10 (ID51), ID 52 through ID 57 should be excluded from analysis. 


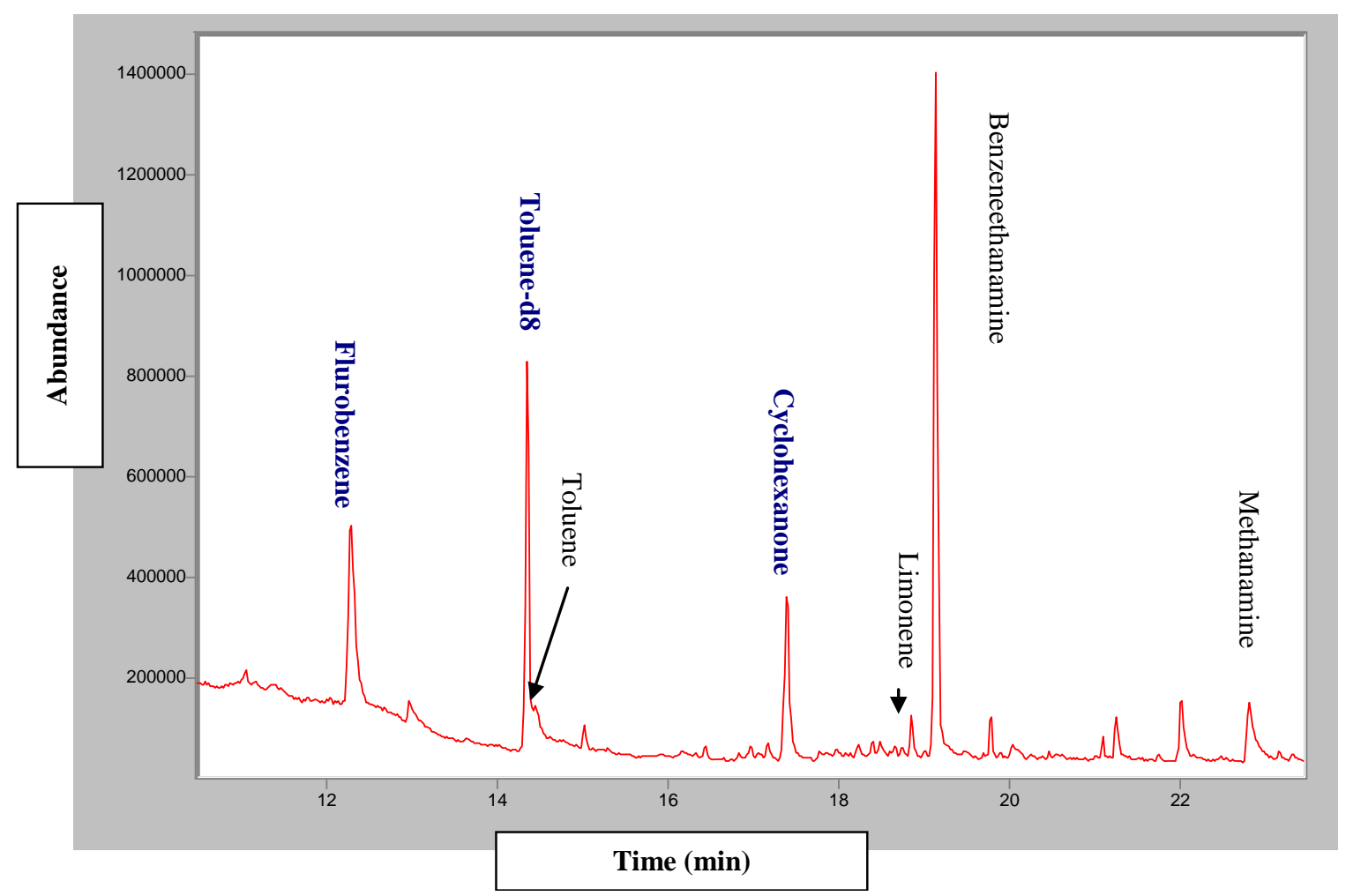

Fig. 6. Representative gas chromatogram showing 3 internal standards used in Phase 2.

\section{Representative analytical laboratory results}

Figure 6 presents a typical gas chromatogram, including the three internal standards used in Phase 2 and several representative VOCs for illustration, obtained from a participant in Phase 2 testing. The standards (fluorobenzene, toluene-d8, and cyclohexanone) are noted in blue. Spectral components (VOCs) in a chromatogram are separated in the form of peaks as a function of retention time in the GC column. The quantity of each VOC component present is indicated by the area under the individual peak associated with the VOC.

Results of laboratory analyses of samples obtained in Phase 1 and Phase 2 identified and quantified 18 VOCs. These are listed in Table 4 and discussed in the following sections. Note that in five cases, the chemical names have been shortened for convenience. The VOCs in Table 4 are organized in five groups based on conventional biochemical classifications, i.e., alkenes, alkanes, etc. 


\begin{tabular}{|c|l|l|}
\hline \multicolumn{3}{|c|}{ Table 4. Detected VOC groupings } \\
\hline $\begin{array}{c}\text { Chemical } \\
\text { group }\end{array}$ & \multicolumn{1}{|c|}{ Abbreviated chemical name } & \multicolumn{1}{c|}{ Full chemical name } \\
\hline 1 & Carene, limonene, toluene & 3-carene, limonene, toluene \\
\hline 2 & Alkane, decane, dodecane, nonane & Alkane, decane, dodecane, nonane \\
\hline 3 & $\begin{array}{l}\text { Decanal, heptanal, hexanal, nonanal, } \\
\text { octanal }\end{array}$ & $\begin{array}{l}\text { Decanal, heptanal, hexanal, nonanal, } \\
\text { octanal }\end{array}$ \\
\hline 4 & Benzald, furan & Benzaldehyde, 2-furancarboxaldehyde \\
\hline 5 & Benzene, methan, octadien & $\begin{array}{l}\text { Benzeneethanamine, methanamine, 3,7- } \\
\text { dimethyl-1,6-octadien-3-ol }\end{array}$ \\
\hline Methyl (6-methyl-5-hepten-2-one) was not chemically similar to any of the other VOC groups. \\
\hline
\end{tabular}

\section{Statistical Analysis}

Statistical analysis was implemented in two steps. First, general descriptive analysis techniques, employing graphics, were applied to provide an initial indication of the characteristics of participant response. This information was used to point the analysis toward appropriate statistical techniques that could lead to useful results and meaningful interpretation.

\section{Descriptive analysis results}

The purpose of the descriptive statistical analysis in this study was to investigate whether VOC measurements differed when subjects were truthfully answering neutral questions versus telling directed lies. To minimize sample-to-sample variation and processing variation, data were normalized using fluorobenzene as the internal standard. The basic unit of analysis for each chemical detected was the subject's VOC peak value divided by the corresponding fluorobenzene peak value. For Phase 2 data, a second set of analyses was performed using the subjects' VOC values normalized by cyclohexanone. The results based on cyclohexanone are not presented since they were very similar to the results obtained using fluorobenzene as the internal standard. Analyses based on the standard toluene-d8 were inconclusive, but this was not unexpected since that standard could adjust only for instrument drift.

Figure 7 is an example figure that shows the normalized values for 23 Phase 1 participants for the chemical benzaldehyde. If an individual VOC could be used to detect deception, an 
ideal pattern for suggesting deception detection would be for most of the IDs to have their deception responses, shown in orange, above (or below) the two neutral responses, shown in blue. This figure illustrates a typical situation with respect to data from Phase 1 testing: there is no clearly defined demarcation of deceptive versus neutral responses.

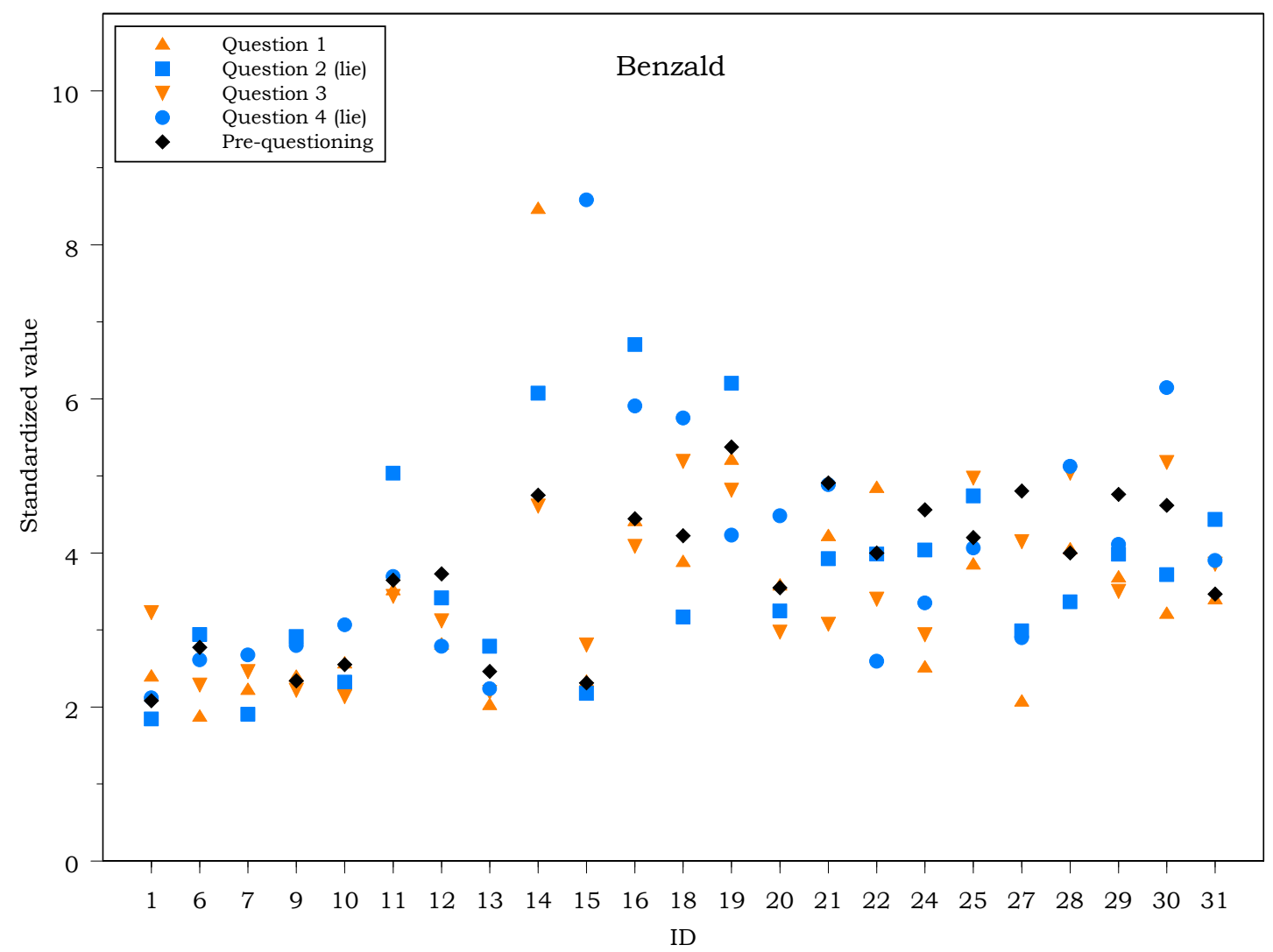

Figure 7. Descriptive presentation of normalized values for benzaldehyde

As shown in Figure 7, responses were not consistently above (or below) the neutral responses for the majority of the IDs for any single VOC during the deceptive question. Therefore, it was decided to employ statistical analysis techniques that would combine the effects of multiple VOCs to examine deception for individual participants.

\section{Statistical analysis methods}

Because the magnitude of the normalized values varied greatly between subjects and the variability of the responses tended to increase with magnitude, a logarithmic transformation 
was applied. The natural logarithms of all the normalized values were calculated, and subsequent statistical analysis was conducted using the logarithms and R software (RDCT, 2004). The difference of two logarithms is mathematically identical to the logarithm of their quotient, so the results are related to percent change in response.

A statistical model was used to adjust for individual VOC differences and provide overall estimates of the effects of the two deception questions relative to the two neutral questions. Specifically, the model used for each subject was:

$$
\begin{aligned}
& \mathrm{y}_{\mathrm{ij}}=\mu_{\mathrm{i}}+\gamma_{\mathrm{j}}+\varepsilon_{\mathrm{ij}} \quad \text { (Equation 1) } \\
& \mathrm{i}=1,2,3, \ldots, \mathrm{n} \text { (n, the number of VOCs) and } \\
& \mathrm{j}=2 \text { if baseline value, or } 3 \text { if deceptive question (the treatment) }
\end{aligned}
$$

where $\mathrm{y}_{\mathrm{ij}}$ is the $\log$ normalized value; $\mu_{\mathrm{i}}$ is the mean response for the $\mathrm{i}^{\text {th }} \mathrm{VOC} ; \gamma_{\mathrm{j}}$ is the difference on the log scale between the neutral question response and the baseline value $\left(\gamma_{2}\right)$ or the deceptive question response value $\left(\gamma_{3}\right)$; and $\varepsilon_{\mathrm{ij}}$ is the error term. Subjects were analyzed first using all VOCs combined and then by each of five groups of chemically similar VOCs. Because the magnitude of the log normalized values varied among the VOCs, an initial step was to adjust these values so that results from multiple VOCs would be weighted equally. For each VOC the overall average among all treatments (baseline, neutral, and deceptive) was determined, and the separate log normalized values were divided by this overall average before applying the model in Equation 1.

For each subject the main parameter of interest is $\gamma_{3}$, which represents the difference between the neutral and deceptive question responses. For example, if:

$$
\gamma_{3}=0.09, \mathrm{e}^{0.09}=1.094 \simeq 1+0.09
$$

this would indicate an approximate 9 percent increase in the deception response over the neutral response. If:

$$
\gamma_{3}=-0.09, \mathrm{e}^{-0.09}=0.914 \simeq 1-0.09
$$

an approximate 9 percent decrease in the deception response compared with the neutral response is indicated. The increase or decrease of the deception response relative to the 
neutral response is defined as the deception relative response $\left(R_{\text {deception }}\right)$. Similarly, the increase or decrease of the baseline value relative to the neutral response is the $\left(R_{\text {baseline}}\right)$. The RRs multiplied by 100 represent the log percent increase or decrease, which closely approximate the percent increase or decrease for values of about 25 percent or less (Tournquist et al., 1985).

\section{Statistical analysis based on all VOCs combined}

For each subject the $R R_{\text {deception }}$, the standard error $(S E)$ of the $R R_{\text {deception, }}$ and the $R_{\text {baseline }}$ based on all VOCs combined are presented in Table 5. Examples found in the figures in Appendices $\mathrm{E}$ and $\mathrm{F}$ include the $\mathrm{RR}_{\text {deception, }}$ its standard error, and a graphical representation of the adjusted values used with the model (in Equation 1) to determine the values of the parameters, including $\gamma_{2}$ and $\gamma_{3}$.

Two types of anomalies could have occurred and been detected in the graphs. One would expect that the Q1 and Q3 responses would be randomly mixed, with some Q1s higher than Q3s and others lower; the same situation should hold for the Q2s and Q4s. The first type of anomaly, which can be called "layers" of responses, occurred when the responses for a treatment (neutral or deceptive) were markedly higher or lower than for the other question of that treatment. The participant's graph should be examined for layered responses-for example, most Q2s on top and most Q4s on the bottom. Such "layering" would suggest that problems occurred during the laboratory analysis of the samples, inadvertently introducing inaccuracies into the data. The second kind of anomaly occurred when some VOCs showed an increase in deceptive over neutral responses while others showed a decrease. The occurrence of this type of anomaly in multiple individuals indicated that for more precise and consistent results, statistics should be calculated for subgroups of these chemicals rather than on all 18 of these chemicals combined. Groups shown in Table 4 would be appropriate. To interpret the results in Table 5 , several points should be considered in determining whether deception could be detected for a participant. Any $\mathrm{RR}_{\text {deception }}$ with a value greater than one (less than one) indicates a general increase (decrease) in response among all VOCs for the deceptive questions versus the neutral questions. However, natural variation is always 
Table 5. Statistical results based on 18 VOCs combined by participant

\begin{tabular}{|c|c|c|c|}
\hline \multicolumn{4}{|c|}{ Table 5. Statistical results based on 18 VOCs combined by participant } \\
\hline Subject & $\mathbf{R R}_{\text {deception }}$ & SE for $\mathbf{R} \mathbf{R}_{\text {deception }}$ & $\mathbf{R R}_{\text {baseline }}$ \\
\hline \multicolumn{4}{|l|}{ Phase 1} \\
\hline ID9 & 31.7 & 9.7 & -6.4 \\
\hline ID10 & 1.9 & 8.8 & -44.3 \\
\hline ID11 & 2.3 & 8.5 & 15.2 \\
\hline ID12 & 6.6 & 4.4 & 20.7 \\
\hline ID13 & 34.6 & 12.9 & 15.1 \\
\hline ID14 & 1.5 & 8.1 & 3.3 \\
\hline ID15 & 53.5 & 14.0 & -8.3 \\
\hline ID16 & -4.7 & 13.8 & 6.2 \\
\hline ID17 (control) & 6.8 & 3.8 & 13.7 \\
\hline ID18 & -15.5 & 12.2 & -26.7 \\
\hline ID19 & -3.5 & 4.4 & 9.5 \\
\hline ID20 & 4.0 & 13.3 & 7.7 \\
\hline ID21 & 26.7 & 6.1 & 16.2 \\
\hline ID22 & -12.6 & 6.4 & -7.6 \\
\hline ID23 (control) & -9.2 & 4.1 & -13.2 \\
\hline ID24 & 17.6 & 5.0 & 9.0 \\
\hline ID25 & -16.6 & 4.4 & -12.8 \\
\hline ID26 (control) & 33.8 & 10.4 & 16.2 \\
\hline ID27 & -3.4 & 12.9 & 19.2 \\
\hline ID28 & -8.4 & 11.4 & -27.5 \\
\hline ID29 & -2.2 & 10.5 & 20.9 \\
\hline ID30 & -7.9 & 6.7 & -5.8 \\
\hline ID31 & -6.7 & 7.1 & -18.9 \\
\hline \multicolumn{4}{|l|}{ Phase 2} \\
\hline ID41 & 9.0 & 5.4 & -28.4 \\
\hline ID42 & 11.7 & 7.9 & 21.9 \\
\hline ID43 & 10.8 & 6.0 & 0.3 \\
\hline ID44 & 15.6 & 5.1 & 17.4 \\
\hline ID45 & 17.4 & 6.0 & 15.3 \\
\hline ID46 & 5.8 & 5.1 & 17.4 \\
\hline ID47 & -1.6 & 7.6 & -34.7 \\
\hline ID49 & -26.6 & 17.2 & 23.2 \\
\hline
\end{tabular}


present in data, and only when the $\mathrm{RR}_{\text {deception }}$ is about twice as large (or more) as its standard error is there evidence of a substantial increase (decrease). In addition, the $\mathrm{RR}_{\text {deception }}$ should differ in magnitude from the $\mathrm{RR}_{\text {baseline, }}$ and the ideal situation is for one to be positive and the other negative. Also, the participant's graph should be examined for consistency in results among the 18 VOCs and for "layers," considering the two types of anomalies previously described.

ID9, ID13, and ID24 (see figures E1, E3, and E7, respectively) demonstrate clear evidence of deception response based on data for all 18 VOCs combined. This response was confirmed by the following:

- The $\mathrm{RR}_{\text {deception }}$ is about three times as large as its standard error

- The $\mathrm{RR}_{\text {baseline }}$ is either negative or much smaller in magnitude than $\mathrm{RR}_{\text {deception }}$

- $\quad$ Q1 and Q3 responses are intermingled

- Q2 and Q4 responses are intermingled

- $\mathrm{Q} 1$ and Q3 neutral responses are generally below the reference line RR=1

- Q2 and Q4 deceptive responses are generally above the reference line RR=1

Possible deceptive responses were also demonstrated by ID21, ID26, ID41, ID42, ID43, and ID45, although analysis by separate chemical groups was required to confirm the outcome. ID11 and ID20 have a large standard error relative to $\mathrm{RR}_{\text {deception. }}$. Figures E2 and E5 show that this large variation comes mainly from one VOC. Therefore, grouped analysis was conducted to examine deception responses revealed by one or more groups. Although ID15 appears to have a strong deceptive response, this outcome is tempered by some evidence of "layering" that can be seen in Figure E4.

Figure 8 is graph E1 for ID9 from Appendix E. The horizontal line through the vertical axis represents an $\mathrm{RR}_{\text {deception }}$ of 1 , for which the neutral and deceptive responses were identical. For each VOC the blue symbols 1 and 3 present the adjusted log normalized responses to the two neutral questions, while the red symbols 2 and 4 show the deception responses. The pattern of many $2 \mathrm{~s}$ and $4 \mathrm{~s}$ above the $1 \mathrm{~s}$ and $3 \mathrm{~s}$ indicates a stronger concentration of the 
VOCs after lying than after answering neutral questions truthfully. The lack of "layering" verifies that the difference is likely to be genuine and not an anomaly of imperfect data.

ID9 Adj Control=NO RR=31.7 SE=9.7

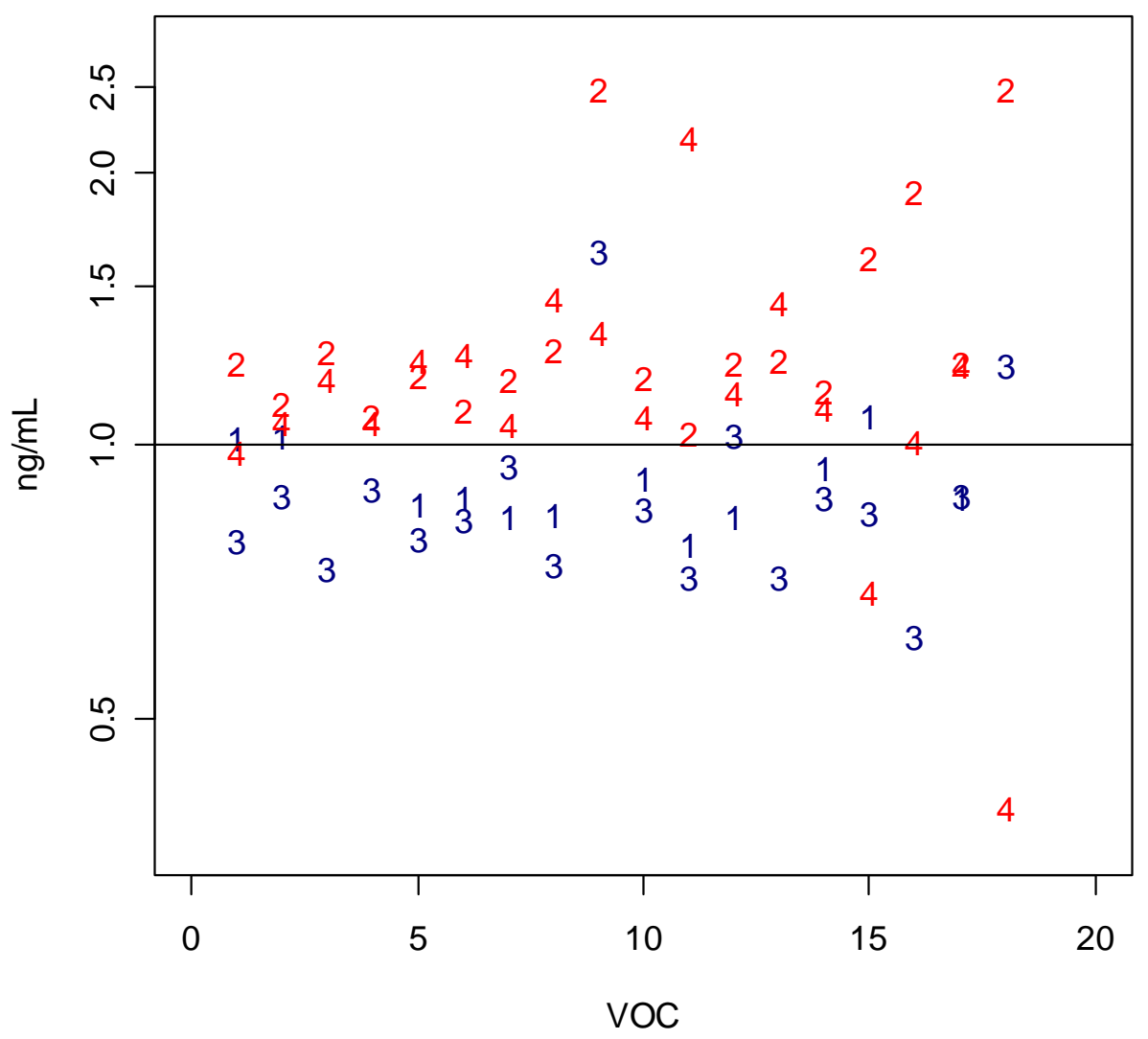

Figure 8. Adjusted normalized values with statistical results for ID9 based on 18 VOCs

\section{Statistical analysis based on groups of chemically similar VOCs}

Although 155 analyses were performed, five for each of the 31 participants suitable for statistical analysis, only selected results of interest are presented in this report. Table 6 contains information identical to Table 5 but is based on data from a separate VOC group, and Appendix F contains a graph for any subject and chemical group for which the elevated $\mathrm{RR}_{\text {deception }}$ was approximately twice its standard error. Because of the limited number of VOCs in a group, it was not possible to check visually for "layering." 


\begin{tabular}{|c|c|c|c|}
\hline \multicolumn{4}{|c|}{ Table 6. Statistical results based on VOC groups by participant } \\
\hline Subject/Group & $\mathbf{R R}_{\text {deception }}$ & $\mathbf{S E}$ for $\mathbf{R R}_{\text {deception }}$ & $\mathbf{R R}_{\text {baseline }}$ \\
\hline \multicolumn{4}{|l|}{ Phase 1} \\
\hline ID9/G2 & 53.8 & 14.0 & 5.2 \\
\hline ID9/G3 & 39.5 & 4.4 & 6.4 \\
\hline ID9/G4 & 22.1 & 4.2 & 5.8 \\
\hline ID11/G4 & 15.0 & 8.3 & -8.7 \\
\hline ID13/G3 & 23.3 & 6.7 & 15.2 \\
\hline ID13/G5 & 145.7 & 69.1 & -2.7 \\
\hline ID15/G2 & 54.8 & 21.3 & -19.9 \\
\hline ID15/G5 & 123.9 & 54.6 & 19.2 \\
\hline ID20/G1 & 15.5 & 4.5 & 10.3 \\
\hline ID20/G2 & 26.3 & 5.4 & -2.2 \\
\hline ID20/G3 & 20.3 & 3.4 & 0.7 \\
\hline ID21/G1 & 19.4 & 8.4 & 21.1 \\
\hline ID21/G2 & 35.8 & 9.2 & 28.6 \\
\hline ID21/G3 & 27.9 & 7.1 & 26.9 \\
\hline ID21/G4 & 19.2 & 9.8 & 26.5 \\
\hline ID24/G1 & 19.4 & 7.0 & 6.7 \\
\hline ID24/G2 & 23.3 & 8.3 & -14.4 \\
\hline ID24/G3 & 14.8 & 6.2 & -0.1 \\
\hline ID24/G4 & 25.2 & 6.5 & 46.8 \\
\hline ID26/G2 (ctrl) & 37.8 & 14.4 & 9.9 \\
\hline ID26/G4 (ctrl) & 53.5 & 25.3 & -1.7 \\
\hline ID26/G5 (ctrl) & 78.4 & 29.1 & 16.9 \\
\hline \multicolumn{4}{|l|}{ Phase 2} \\
\hline ID41/G3 & 18.7 & 8.2 & -2.8 \\
\hline ID42/G3 & 17.5 & 6.9 & -5.1 \\
\hline ID43/G3 & 21.9 & 7.5 & 18.7 \\
\hline ID45/G1 & 15.4 & 7.1 & 31.7 \\
\hline
\end{tabular}

ID9, ID13, and ID24, for whom deception was detected by the combined VOC analysis, were identified as being deceptive by multiple chemical groups. The overall analysis suggested detection of deception for ID21, ID26, ID41, ID42, ID43, and ID45, and results of analysis by chemical group corroborated this finding. In addition, detection of being deceptive was found for ID15 and ID20, who had multiple chemical groups indicating deception, and for ID11, which was identified by one chemical group. Three controls were 
available to investigate the possibility of false positive results, (i.e., detecting deception where none existed). Two of the three controls had no difference between neutral and deception responses either for the combined VOC analysis or for any chemical group analysis. However, data for ID26 indicated a false positive detection of deception.

\section{Findings}

A major finding in this study was that, in general, participant responses to stress induced by deceptive behavior registered an increase in VOC emission level compared to neutral conditions. In the earlier study conducted by CHRS on VOC emissions, individuals watched a video of a violent situation (Watkins et al., 2005). Results showed markedly decreased emission levels in several VOCs. A Japanese study (Tanaka and Ide, 1998) using infrared thermography showed facial skin surface temperature increased in response to emotions including sadness, joy, and anger, but found that temperatures decreased during fear. Since fear was not the dominant emotional stressor in the current study, the results are directly in line with those of the Japanese study.

\section{Discussion and Interpretation of Results}

This research was the first attempt to investigate using the GC/MS to identify deceptive responses from participants involved a realistic testing scenario combined with a shortened sampling time interval. Therefore, Phase 1a, which included the first eight participants, was a pilot test of the equipment and scenario. Analysis of data from these participants determined that one-minute sampling interval was insufficient to yield meaningful data because spectral peaks for several of the VOCs were not distinct enough to provide accurate results.

Therefore, starting with Phase $1 \mathrm{~b}$ the sampling time interval was doubled to two minutes, which provided the distinct peaks needed for more accurate data analysis.

When data from Phase $1 \mathrm{~b}$ and $1 \mathrm{c}$ were examined, it was found that for some subjects the magnitude of the responses appeared to be associated with the corresponding question in a non-random manner. For example, a pattern might be seen in most of the GC/MS data for the 18 VOCs where the question 4 spectral peak values were highest, those for question 1 were next highest, question 3 were next, and question 2 were lowest. This would be in 
contrast to detecting deception reliably, where question 4 and question 2 values would be intermingled and question 3 and question 1 values would be intermingled at a consistently lower (or higher) level. The lack of consistency in the results prompted a thorough investigation of the laboratory equipment and data collection methods. It was found that some improvement could be made in stabilizing the airflow rate through the sampling apparatus, and this change was made for the collection of Phase 2 data. In an effort to further improve the quality of the data, additional internal standard VOCs were adopted to allow comparative analyses.

\section{Question treatments*}

Question 1: Neutral

Question 2: Deception

Question 3: Neutral

Question 4: Deception

* See Appendix A for the content of each question.

In addition to the commonly used internal standard compound fluorobenzene, Phase 2 used two other internal standard organic chemicals, cyclohexanone and toluene-d8, to allow testing instrument variability effects on the data. Besides incorporating the additional internal standards to assess variability in the data, tighter control was maintained over the flow rate through the test apparatus to minimize variability.

Phase 2 utilized data from a total of 15 participants. Unfortunately, the GC/MS instrument malfunctioned during the sample processing. Responses for the first five participants were acceptable, however the data for the remaining ten subjects in Phase 2 were questionable. Based on statistical methods using the fluorobenzene and cyclohexanone data, it was determined that data from the last five participants was compromised by the malfunction and unusable. Therefore, these participants were not included in the statistical analysis. Although it could not be verified that data from the middle five participants were questionable, it may be that the GC/MS results were gradually deteriorating and data for these subjects were not completely accurate and reliable and were not of the same quality as the first five. Data were not available for all VOCs from the last two of the middle five participants; therefore, data from these two individuals were not included in the statistical analysis. Use of multiple internal chemical standards, which provided information to distinguish between expected sample variation and the effects of unexpected instrument and equipment malfunction proved to be distinctly valuable in identifying questionable data. 
The unfortunate instrument malfunction affected the amount of high quality data obtained in this study. However, had the equipment failure not occurred during the Phase 2 sample analysis, the clear results seen in the first five Phase 2 participants could very well have been maintained.

Another achievement of this study was development of reliable statistical analysis techniques and software for combining data from multiple questions and multiple VOCs. The usefulness of these techniques depends on three factors: (1) acquiring accurate and reliable VOC samples, (2) obtaining accurate and reliable laboratory analysis data, and (3) developing a realistic scenario to ensure that participants in the research experience genuine emotional stress responses to test stimuli. Meeting these three conditions is necessary for obtaining clear indications of deception. Unfortunately, in any study where subjects are simulating a real-life situation, one cannot guarantee that participants actually experienced the sufficiently heightened responses necessary to demonstrate unequivocally positive interpretation of the results. Fortunately, the problems associated with instrumentation malfunction and imprecision can be addressed successfully with introduction of multiple internal standard VOCs and continued analytical procedure improvements. Nonetheless, valuable knowledge and practical experience in the application of GC/MS techniques for detecting deception has been acquired and can be further expanded and effectively used in future studies.

\section{Conclusions}

Results of the earlier study confirmed that VOCs can be used to measure responses to intense emotions in certain individuals. In that study, emotional responses were identified in participants who watched a video of an actual workplace violence incident. Data were collected during the entire prolonged sampling interval (5 min) compared to the shorter (2 min) interval in this study, which included a more realistic and involved scenario. However, in this study, sustained emotional involvement by participants could not be ensured even though every effort was made to make the scenario effective. Furthermore, even for those that experienced a genuine stress response to the scenario, the fact that the deception was carried out by simply replying to a single question with a one-word answer may have truncated the length of meaningful response. The data collection period following each question was only 40 percent as long as that in the earlier study. Also, the type of emotional 
response elicited when watching a video depicting violence may likely differ from the emotions experienced while lying to a question.

It was concluded that, when responses are not intense, it is necessary to focus on groups of chemically similar VOCs rather than individual chemicals to more readily indicate deception. Also, using groups of similar chemicals rather than all VOCs combined provides increased sensitivity and resolution in mass spectrometry results to indicate deception.

To achieve meaningful results, two conditions had to be met: (1) the participant had to have a genuine response to lying; and (2) the analytical data had to be accurate. Numeric and graphical statistical analysis were successful in identifying deception in a number of subjects, thus these conditions were met to some degree.

It is evident from the results of this study that work needs to continue on developing a testing scenario that causes emotional response to deception by most or all participants. It would also be helpful to obtain and incorporate into the exit interview each participant's quantitative self-measure of emotional involvement in the scenario.

Like the earlier study, much has been learned and further research should be conducted based on the encouraging results achieved in this and the previous study. Meaningful research is at times not a fast-paced process, and equipment malfunctions do unfortunately occur. However, the goal of achieving a viable, accurate, and reliable alternative to the traditional polygraph is a worthy goal that should not be abandoned. When developed, VOC monitoring technology shows promise in providing a reliable supplementary or alternative technology to the polygraph that can help reduce the negative impacts of the polygraph and lead to greater acceptance of psychophysiological testing of personnel who have access to sensitive national security information.

\section{Recommendations}

It is recommended that future work include the following:

- Expand the array and obtain more comprehensive characterization of the VOCs emitted by human subjects. Previous studies revealed up to 14 relevant VOCs given off by humans under stress. This study investigated a total of 18 . 
- Continue work to develop a more effective testing scenario by partnering with the Department of Defense Polygraph Institute and incorporating this technology with their current polygraph training program.

- Continue to investigate, adapt, and assess feasibility of using emerging VOC sensor technologies to enhance detector capability to capture changing VOC concentrations in response to induced stress levels.

- Acquire and employ more advanced sampling and analytical methodologies.

- Continue research to determine whether SVOCs can represent an additional viable means to detect deception. 


\section{References}

Asano, K. G., C. K. Bayne, K. M. Horsman, and M. V. Buchanan. 2002. "Chemical Composition of Fingerprints for Gender Determination.” Journal of Forensic Science, Vol. 47, No. 4.

Bernier, U. R., D. L. Kline, D. R. Barnard, C. E. Schreck, and R. A. Yost. 2000. “Analysis of Human Skin Emanations by Gas Chromatography/Mass Spectrometry. 2. Identification of Volatile Compounds that are Candidate Attractants for the Yellow Fever Mosquito (Aedes aegypti)." Analytical Chemistry, Vol. 72, pp. 747-756.

Ellin, R. I., R. L. Farrand, F. W. Oberst, C. L. Crouse, N. B. Billups, W. S. Koon, N. P. Musselman, and F. R. Sidell. 1974. "An Apparatus for the Detection and Quantitation of Volatile Human Effluents." Journal of Chromatography, Vol. 100, pp. 137-152.

National Research Council. 2003. The Polygraph and Lie Detection. Committee to Review the Scientific Evidence on the Polygraph, National Academy of Sciences, Division of Behavioral and Social Sciences and Education. National Academies Press: Washington, DC.

RDCT (R Development Core Team). 2004. R: A Language and Environment for Statistical Computing. ISBN 3-900051-00-3, R Foundation for Statistical Computing, Vienna, Austria (available at http://www.R-project.org ).

Tanaka, H., and H. Ide. 1998. "Emotion Analysis Using Facial Skin Thermogram.” Proceedings of the Symposium on Biological and Physiological Engineering, Vol. 13, pp. 533-536.

Tournquist, L., P. Vartia, and Y. O. Vartia. 1985. "How should relative changes be measured?" American Statistician, Vol. 39, pp. 43-46.

Watkins et al. 2005. "Detection of Deception Using Volatile Organic Compound (VOC) Emissions." Final Report, Center for Human Reliability Studies, Oak Ridge Institute for Science and Education, Report Number 05-0330.

Zhang, Z-M., J-J. Cai, G-H. Ruan, and G-K. Li. 2005. "The study of fingerprint characteristics of the emanations from human arm skin using the original sampling system by SPME-GC/MS." Journal of Chromatography B, Vol. 822, pp. 244-252. 


\title{
Appendix A: Testing Protocol and Scenario
}

\section{TESTING PROTOCOL AND SCENARIO}

\author{
Research Study: Extended Research on Detection of Deception Using Volatile \\ Organic Compound (VOC) Emissions
}

Step 1: After arriving and obtaining a temporary badge, the participant is escorted into the interview room to complete the consenting process.

\section{Note:}

- This scenario incorporates only one deception item to ensure sufficient statistical power to determine true positive results.

Step 2: The participant completes the consenting process and receives instructions. After consenting, the participant is escorted into another room to pick up the handgun (to lie about possessing) in the presence of the uniformed police officer and then proceeds into the testing room carrying the handgun in a bag accompanied by the police officer.

Step 3: In the testing room the participant can view the test equipment and ask questions. The data collection system is explained and the subject is situated for testing.

Step 4: The following 4 questions are asked in sequence with a 2.5 min data sample taken after each question is asked. The first sample, however, is for baseline data with no question asked (the first of 5 samples per person).

- $\quad 1^{\text {st }}$ neutral Q: (1) "Is today Friday?"

Answer truthful "yes" or "no"

(2) "Are you sure that today is not Friday?"

- $\quad 1^{\text {st }}$ relevant Q: (1) "Did you take the handgun?"

(2) "Is the handgun in the bag?"

o Directed lie

Answer "no"

- $\quad 2^{\text {nd }}$ neutral Q: (1) "Is today Tuesday?"

Answer truthful "yes" or "no"

- $\quad 2^{\text {nd }}$ relevant Q:

(2) "Is today Tuesday?"

(1) "Do you know where the handgun is?"

(2) "Is the handgun in the bag?"

o Directed lie

Answer "no" 


\section{Testing Protocol and Scenario}

Page 2

\section{Notes:}

1. Data collection will commence when the researcher asks a question.

2. The participant will be instructed to wait 5 to $10 \mathrm{sec}$ before answering the question.

3. Also, during the 2.5 min sampling interval, after another 5 to $10 \mathrm{sec}$ have elapsed beyond the participant's answer, the researcher will ask the question a second time (worded slightly differently) to ensure participant focus and that the question was understood.

4. The participant will be instructed again to wait 5 to $10 \mathrm{sec}$ before answering the second time for each question.

\section{Step 5:}

After testing, the apparatus is disconnected, samples sealed, and the samples are sent to the laboratory for analysis.

\section{Step 6:}

Following testing, the subject is debriefed and asked how he/she felt during the testing experience.

\section{Other Notes:}

1. An armed police officer will be present when the subject picks up the handgun to lie about during testing. This will heighten realism of the scenario.

2. A total of 16 subjects will be recruited for Phase 2 of this study.

3. Participants will be offered $\$ 50$ compensation for their involvement.

4. At the end, when disbursing compensation:

a. Inform the participant that the test scenario was a simulation and that the deception item was, in fact, a prop.

b. Be sure to caution participants not to tell anyone about the testing scenario so as to not bias a subsequent participant. 


\title{
Appendix B: Informed Consent Form CONSENT FOR RESEARCH STUDY
}

\author{
Principal Investigators: $\quad$ G. R. Eisele, Ph.D. and D. R. Watkins, Ph.D., Center for \\ Human Reliability Studies, Oak Ridge Institute for Science \\ and Education, Oak Ridge, Tennessee 37830, Telephone:
} (865) 576-2208

$\begin{array}{ll}\text { Title of Project: } & \frac{\text { Research Study: Extended Research on Detection of }}{\text { Deception Using Volatile Organic Compound (VOC) }} \\ & \underline{\text { Emissions }}\end{array}$

\section{Purpose of this study}

You are being asked to voluntarily participate in a research study. The objective of this study is to determine the relationship between small changes in natural organic bodily chemicals emitted from the skin surface of the forearm and the physiological responses related to deceptive stress. This study is being sponsored by the National Security Agency.

\section{Procedures to be followed}

In this study you, the participant, will be asked to sit at a table in the testing facility. You will have your forearm resting in a comfortable position in a plastic sleeve on a table and air samples will be drawn off and collected in sample collection tubes to obtain data on the natural volatile organic compounds (VOCs) contained in the air coming off your skin. The purpose is to determine if changes in VOCs could be used to indicate the presence of intentional deception or lying by people.

The first thing that you will be asked to do is to select a folded piece of paper from a container. Then you will be asked to review and sign the consent form to indicate that you want to voluntarily participate in the study.

Following the consenting process, if you selected a piece of paper with the word "item" written on it, you will be asked to step into another room. In the other room you will be asked to take a piece of criminal evidence obtained during a law enforcement drug sting operation. The item is a double-wrapped bag of an illegal drug. After you pick up the item in the presence of an armed guard, you will put it into a carrying case and be escorted to the testing room for sample data collection. You will be directed to lie later about having this item in your possession. 
Appendix B (continued)

CONSENT FOR RESEARCH STUDIES

Skin Emissions Deception Detection Project

Page 2

In the testing facility you will meet the researchers, be shown the equipment setup, and have the opportunity to ask questions about the setup before testing begins.

The researchers in the testing room will not be present when you take the item and will have no prior knowledge of the item you took. During testing and data collection, you will be asked a single series of four questions that will include relevant statements about the item you have in your possession as well as some irrelevant questions. As instructed, you are directed to lie when answering questions related to the particular item you have. After each question is asked, you will wait 5 to 10 seconds before answering the question, and you are to answer all questions directly with only a simple "yes" or "no" using an even speaking level and tone of voice after the question is asked.

After you answer each question the first time, 5 to 10 seconds later and while data are being taken, the researcher will ask the same question a second time to ensure that you fully understand the question and answer you give. All questions will respect your privacy and will not include any questions involving personal matters, such as lifestyle, legal matters, or personal habits. No other questions or other verbal response will be asked of you during the entire testing process.

Air and VOC samples will be collected in sampling tubes for 1.0 minute after each question is asked with a brief rest period between questions. There will be no contact between the sampling apparatus and you except for the sampling sleeve. You will be asked to hold your arm relatively still in a comfortable position while sampling. The forearm will be enclosed in a loose fitting, open-ended, inert plastic sampling sleeve with a sampling port built into the side of the sleeve. After testing, the sampling tubes will be stored and sent to the laboratory for analysis to determine the relationship between natural VOCs from your forearm and your responses.

If you experience any discomfort during data collection, every reasonable effort will be made by the researchers to accommodate your needs.

Compensation in the amount of $\$ 50$ in cash will be offered to you once you have completed all data collection requirements. If you stop your participation before all data are collected, you will not receive any money for your participation.

Test data will not be personally identifiable at any stage of this study. No personally identifiable information on subjects will be retained, attached, or linked to test data at any time. There will be need, however, to retain the original signed consent forms to meet regulatory requirements and the signed compensation receipt forms to satisfy ORAU requirements, but these will not be linked in any way to test data or study results. As soon as practical after the data have been analyzed, any possible identifying information about the participants will be destroyed. All test data and the signed consent and cash receipt forms will be kept in secure, locked cabinets in separate locations. 
Appendix B (continued)

CONSENT FOR RESEARCH STUDIES

Skin Emissions Deception Detection Project

Page 3

No information gathered in this study will be made available to law enforcement officials or any other officials unless dictated by legal proceedings. If you have any concerns about this type of legal disclosure, then you should consider not participating.

Following data collection, you will receive a short debriefing and will be asked how you felt during the testing process.

\section{Duration of this study}

Your participation in this study will take approximately $1 / 2$ hour of your time.

\section{Discomforts, inconveniences, and/or risks that can be reasonably expected}

Discomforts - If you have back pain or other conditions that might interfere with your ability to sit for as long as $1 / 2$ hour, you may not want to participate in this study. Should you have any discomfort, tell the researcher, and you will be able to take a break. If there is actual pain involved, you will be excused immediately. No other discomforts are known or anticipated.

Inconveniences - Because of the length of the study, you may be inconvenienced by your participation. This might interfere with your personal/professional/educational schedule. Should this occur, tell the researcher immediately, and he will make all reasonable attempts to eliminate the inconvenience. No other inconveniences are known or anticipated.

Risks - The researchers are unable to guarantee that your identity will not be disclosed to other persons or organizations. Therefore, if you have any reason to withhold your identity from outside sources (including the sponsoring agency), you should not participate in the study. The researchers are similarly unable to ensure that other persons, following legal proceedings (such as the use of subpoenas or other legal discovery instruments), will not be able to identify you as a subject in the study. However, to the fullest extent possible, the researchers will protect your identity.

There will be physical contact of the sample collection system with your forearm. There is no known risk of exposure to any toxic materials in this study. There is also no known risk of other harmful exposures associated with this study.

\section{Potential benefits of the study}

Because the study is designed to assess the feasibility of using this technology in government security programs, there are no anticipated benefits to you as an individual. This is not a treatment for any medical or other condition but, rather, only represents research to determine the relationship of variations in natural organic chemical emission levels (VOCs) from your skin to heightened bodily responses that could be related to lying. 
Appendix B (continued)

CONSENT FOR RESEARCH STUDIES

Skin Emissions Deception Detection Project

Page 4

\section{Your rights as a participant}

Your participation in this study is entirely free and voluntary. You have the right to refuse to participate or to withdraw from the study at any time. However, to receive compensation you must complete the data collection requirements.

This form provides information to you about this study and your participation in it. You have been asked to read this form carefully. All your questions about the study, the procedures required to do the study, and the information provided on this form have been answered by (name) at (865) 576-2208.

If you need more information about this study at any time, you may contact Dr. G. R. Eisele or Dr. D. R. Watkins at (865) 576-2208. If you need more information about your rights as a study participant, you may contact the Chairperson of the Oak Ridge Site-wide Institutional Review Board at (865) 576-1725.

\section{To persons who agree to participate in this study}

I have read that if I have further questions or need more information, I can contact the above personnel at any time. I have read the purpose of this study, the procedures involved, and the possible risks and benefits.

I have been told that all information obtained in the study will be the property of the sponsor.

In the event that results of this study are published in the open literature, I would (check one) YES (I want to) NO (I do not want to) receive a summary of the study results.

\section{I have been informed that I may decline to participate in the study. I freely and voluntarily choose to participate.}

I, (Print/type Name of Volunteer) voluntarily agree to participate in this study and know that I may withdraw at any time.

I know that I can receive a copy of this consent form, if desired, after it is completed. All of my questions about the study and my participation in it have been answered to my satisfaction. I know that if I have questions in the future, I can contact Dr. G. R. Eisele or Dr. D. R. Watkins at (865) 576-2208 or the Chairperson of the Oak Ridge Site-wide Institutional Review Board at (865) 576-1725. 
Appendix B (continued)

CONSENT FOR RESEARCH STUDIES

Skin Emissions Deception Detection Project

Page 5

Date:

$\overline{\text { Signature of Volunteer }}$

$\mathrm{I}$,

(Print/type Name of Person Obtaining Consent) have explained and discussed the above information with (Print/type Name of Volunteer) and have answered all questions to his/her satisfaction.

Signature of Person Obtaining Consent/Date 


\section{Appendix C: Recruiting Flyer}

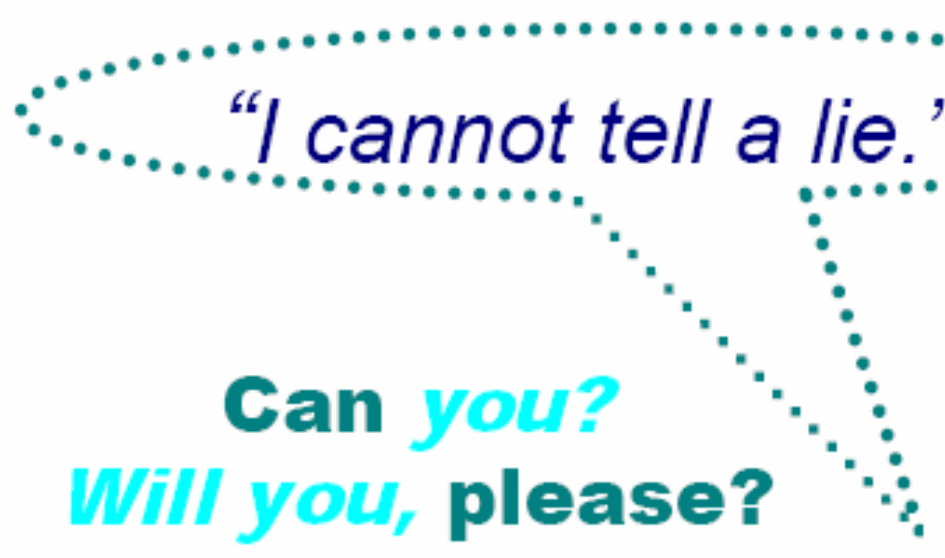

We're looking for volunteers for a research project testing a new way to detect lying.

-PRIVATE AND CONFIDENTIAL

-FLEXIBLE SCHEDULING

\section{What we need...}

\section{About one hour of your time.}

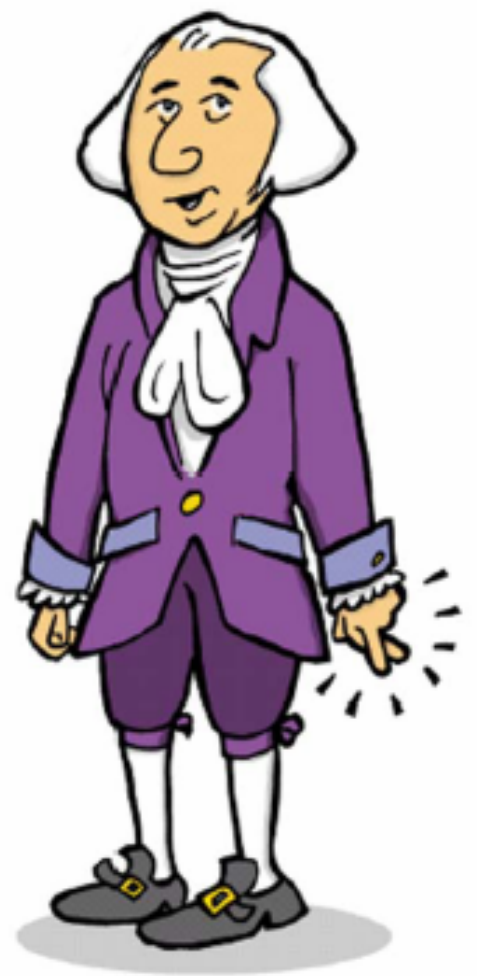

You will be asked nonpersonal questions. Some you will answer truthfully, but you will lie when you answer others. Not everyone who volunteers will be selected.

\section{Participants will be compensated}

If you'd be interested in "lying for science," contact:

Center for Human Reliability Studies

Oak Ridge Institute for Science and Education

(865) 576-2208

\section{O R I S E}




\section{Appendix D: Participant Demographic Data}

\begin{tabular}{|c|c|c|c|c|c|c|c|}
\hline \multicolumn{8}{|c|}{ Phase 1a, 1b, and 1c Testing Groups } \\
\hline & $\begin{array}{c}\text { No. of } \\
\text { participants }\end{array}$ & $<\mathbf{2 5}$ & $\mathbf{2 5 - 3 4}$ & $\mathbf{3 5 - 4 4}$ & $\mathbf{4 5 - 5 4}$ & $\mathbf{5 5 +}$ & $\begin{array}{c}\text { Racial } \\
\text { group }\end{array}$ \\
\hline Male & 16 & 4 & 3 & 3 & 1 & 5 & 16 White \\
\hline Female & 16 & 4 & 2 & 6 & 3 & 1 & $\begin{array}{l}\text { 1 Hispanic } \\
\text { 15 White }\end{array}$ \\
\hline Total & 32 & 8 & 5 & 9 & 4 & 6 & $\begin{array}{l}\text { 1 Hispanic } \\
\text { 31 White }\end{array}$ \\
\hline
\end{tabular}

\begin{tabular}{|c|c|c|c|c|c|c|c|}
\hline \multicolumn{9}{|c|}{ Phase 2 Testing Group } \\
\hline Male & $\begin{array}{c}\text { No. of } \\
\text { participants }\end{array}$ & $<\mathbf{2 5}$ & $\mathbf{2 5 - 3 4}$ & $\mathbf{3 5 - 4 4}$ & $\mathbf{4 5 - 5 4}$ & $\mathbf{5 5 +}$ & $\begin{array}{c}\text { Racial } \\
\text { group }\end{array}$ \\
\hline Female & 9 & 0 & 3 & 3 & 1 & 0 & $\begin{array}{l}\text { 1 Hispanic } \\
\text { 6 White }\end{array}$ \\
\hline Total & 16 & 0 & 4 & 2 & 3 & 0 & $\begin{array}{l}\text { 1 Black } \\
\text { 8 White }\end{array}$ \\
\hline
\end{tabular}




\section{Appendix E. Statistical Results Based on 18 Vocs}

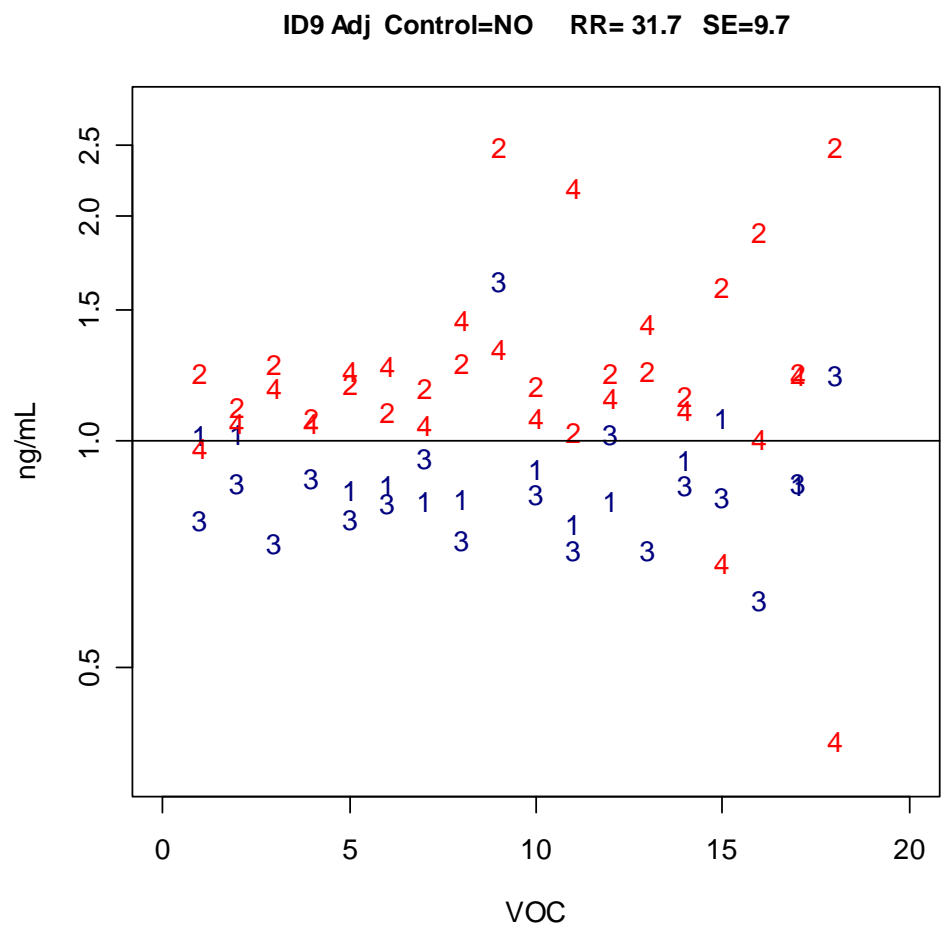

Figure E1. Statistical results for ID9

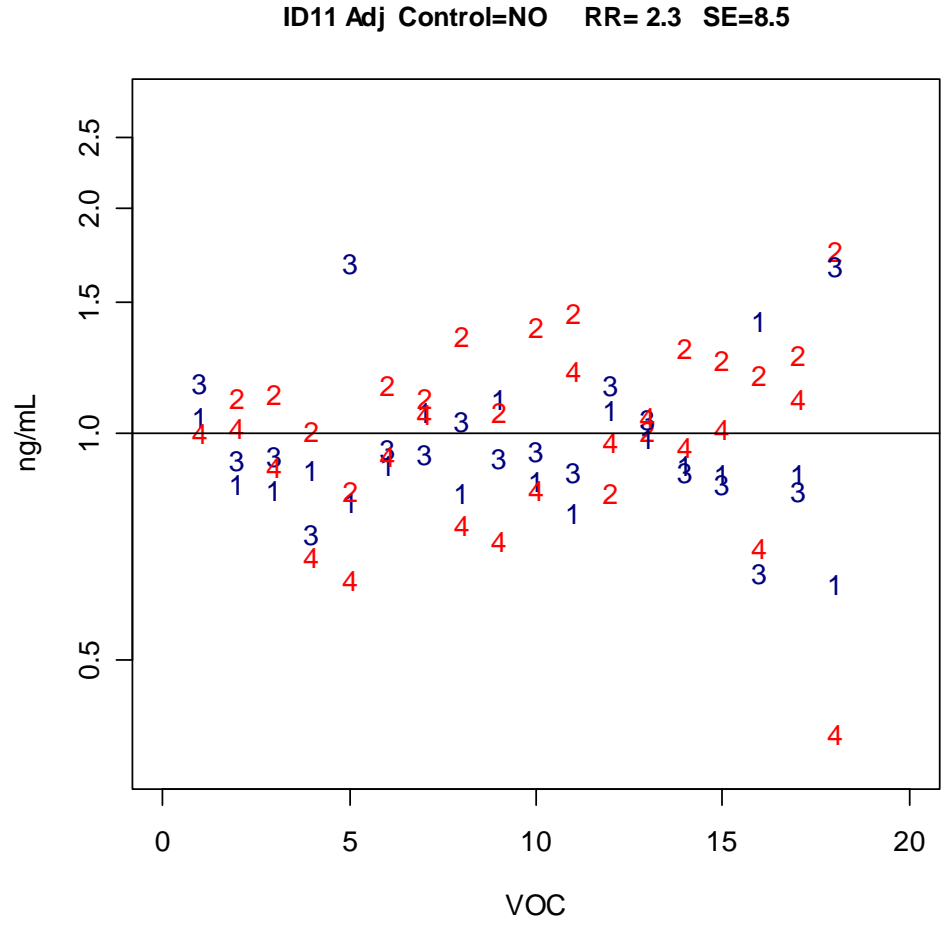

Figure E2. Statistical results for ID11 


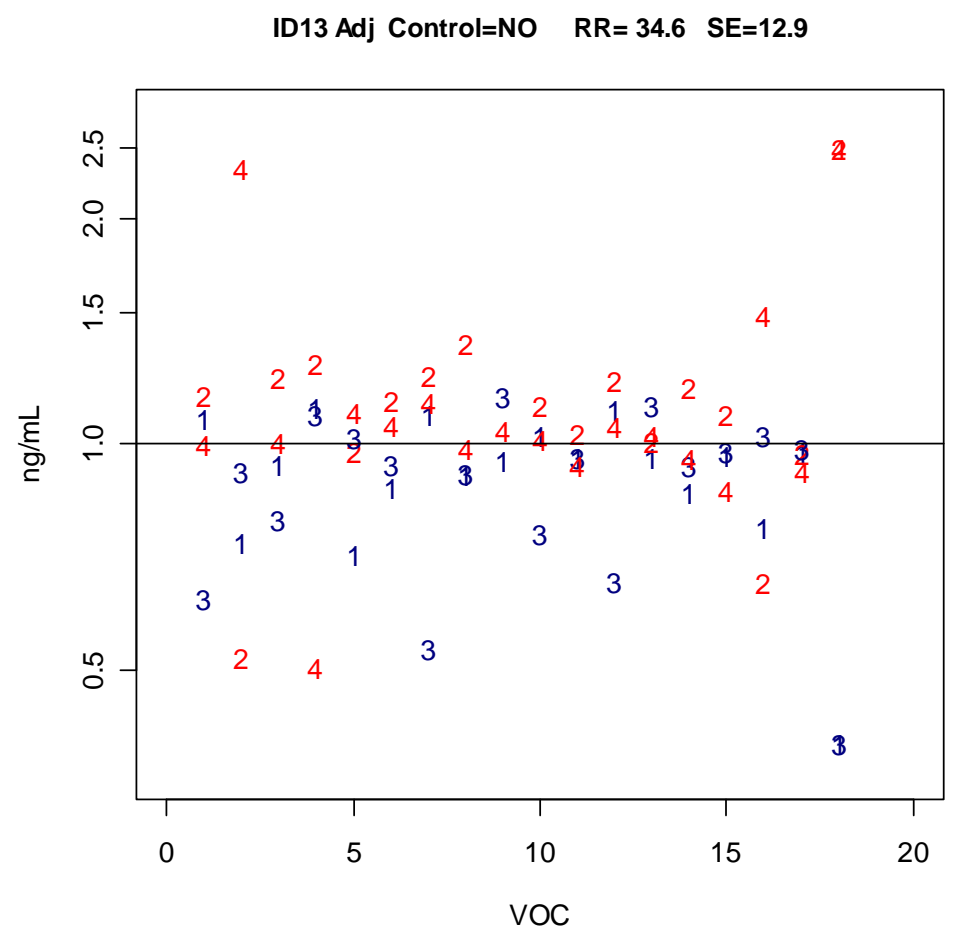

Figure E3. Statistical results for ID13

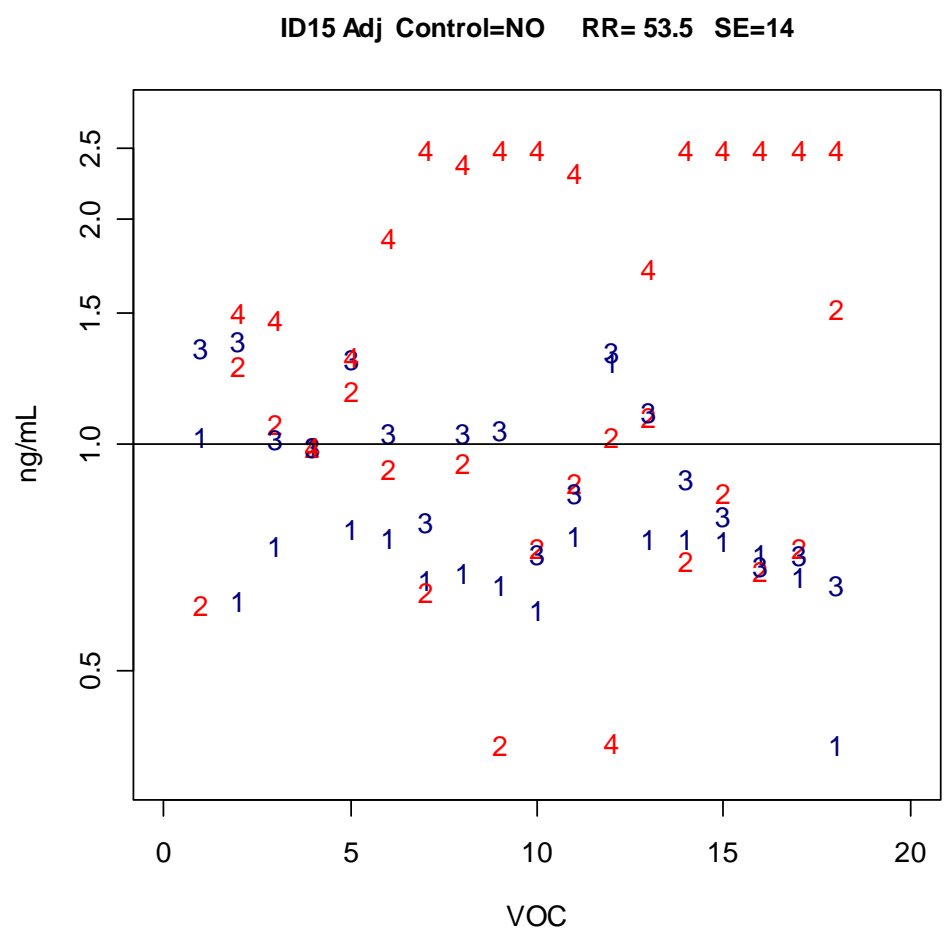

Figure E4. Statistical results for ID15 


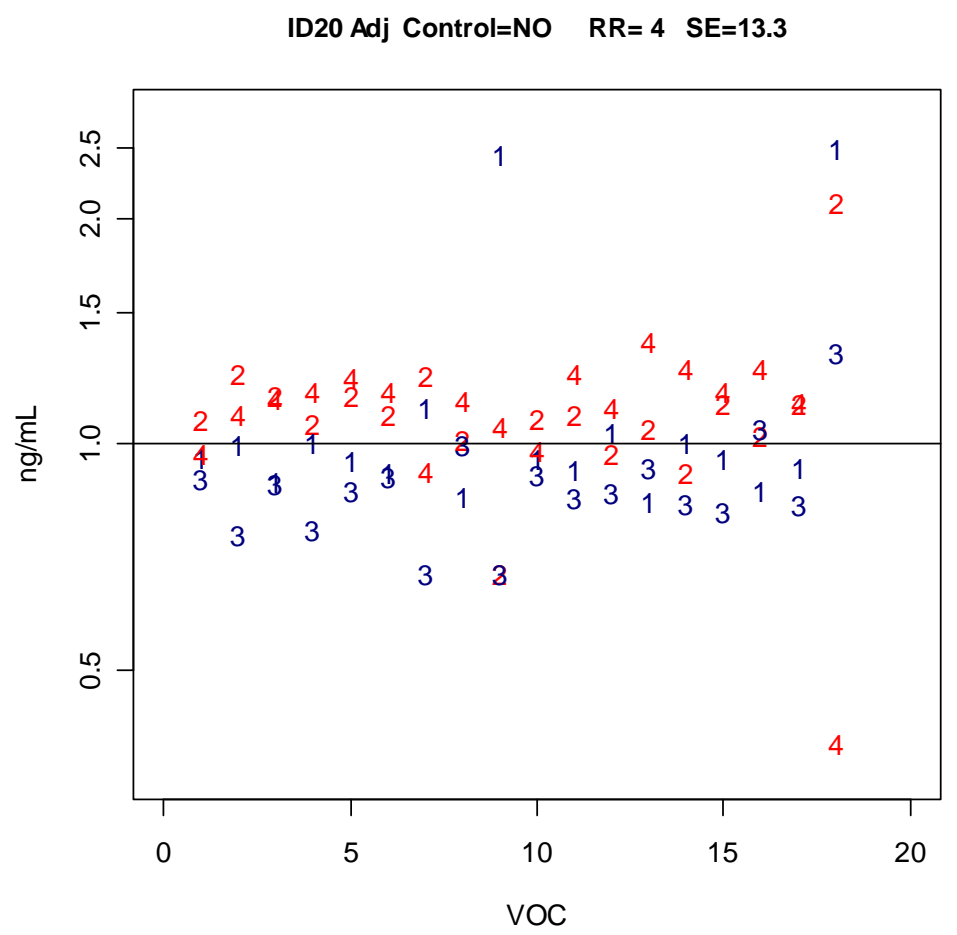

Figure E5. Statistical results for ID20

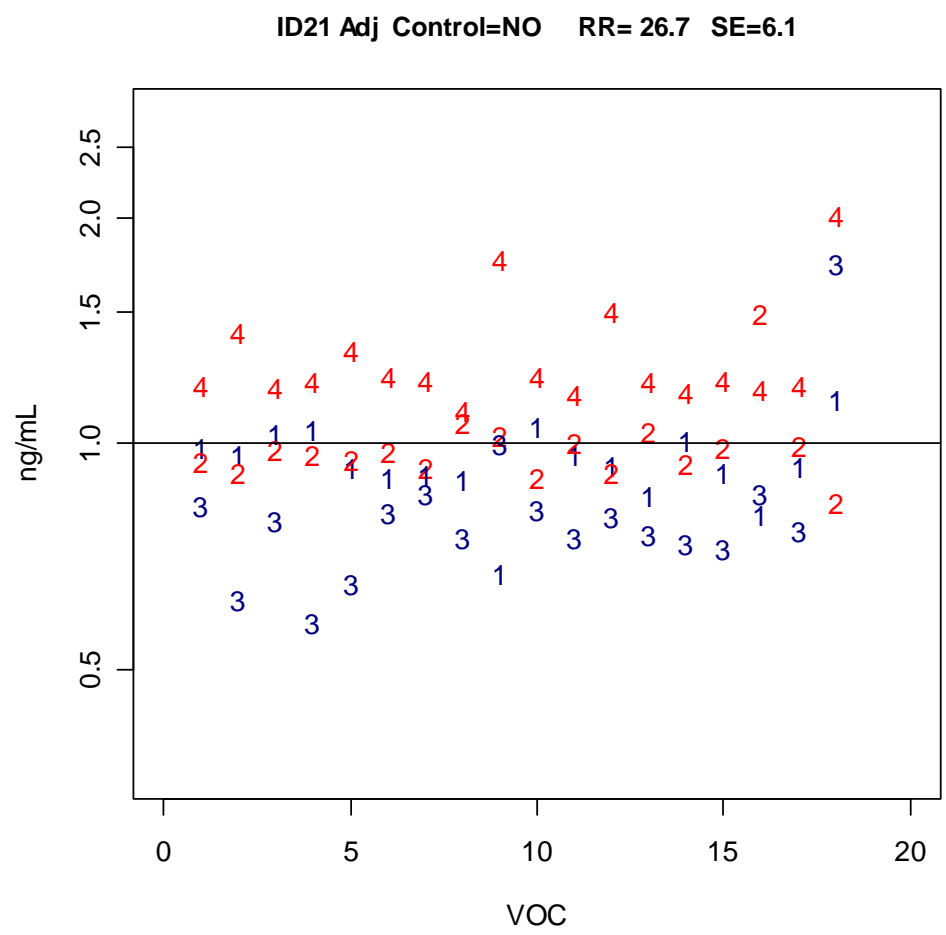

Figure E6. Statistical results for ID21 


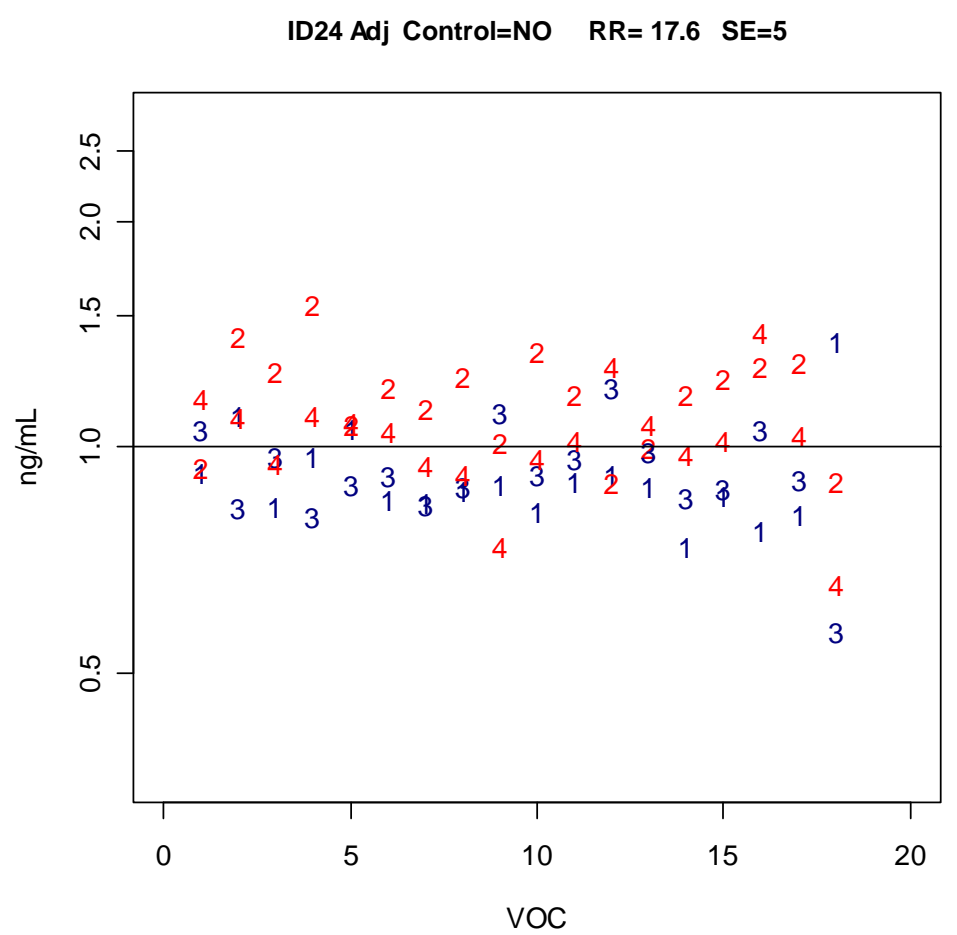

Figure E7. Statistical results for ID24

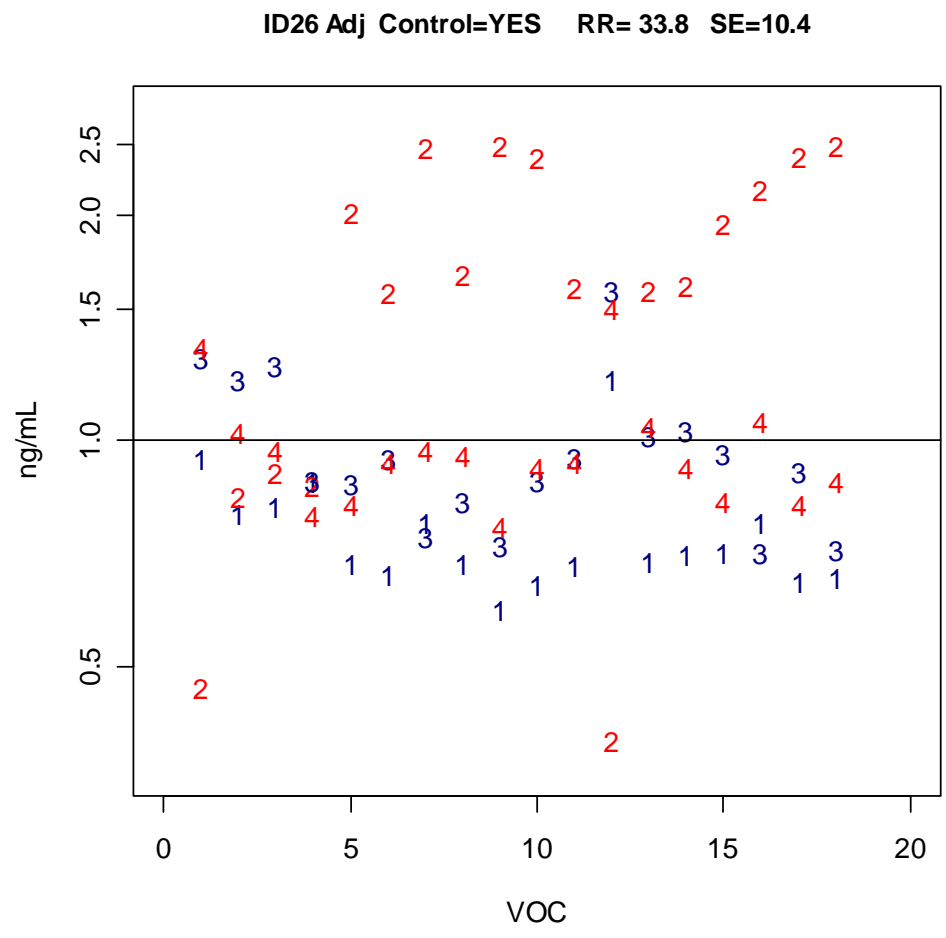

Figure E8. Statistical results for ID26 


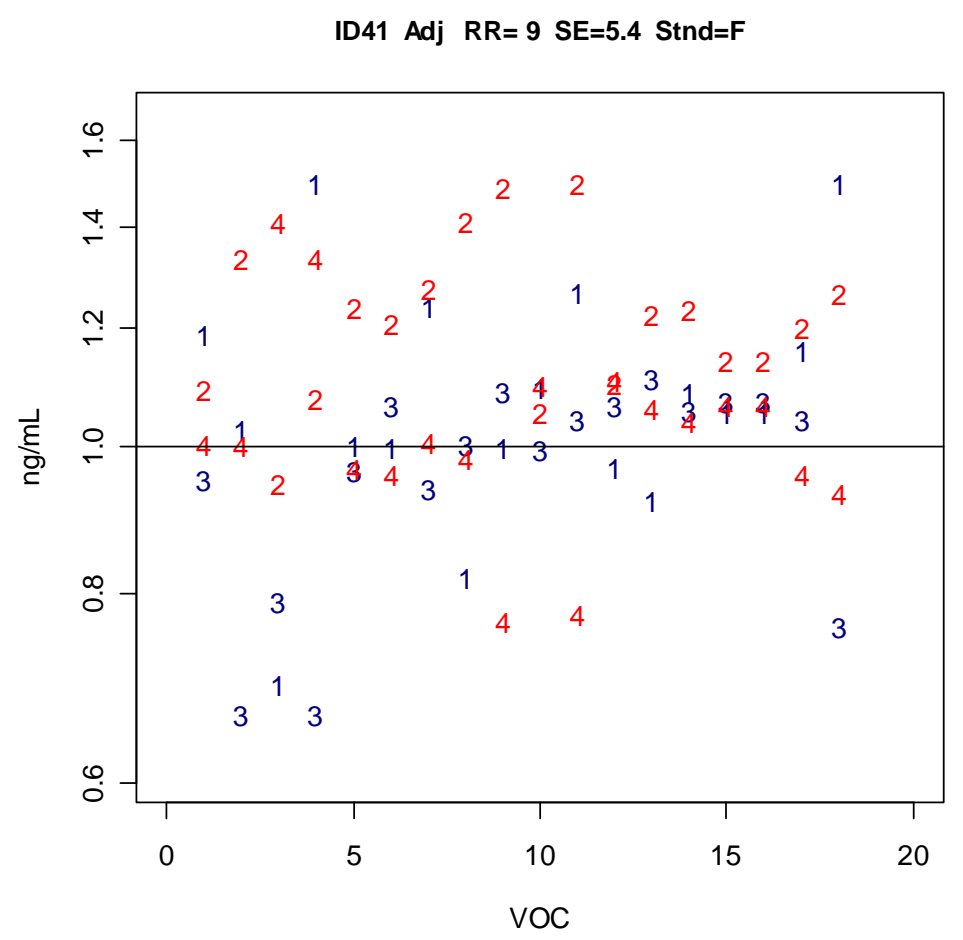

Figure E9. Statistical results for ID41

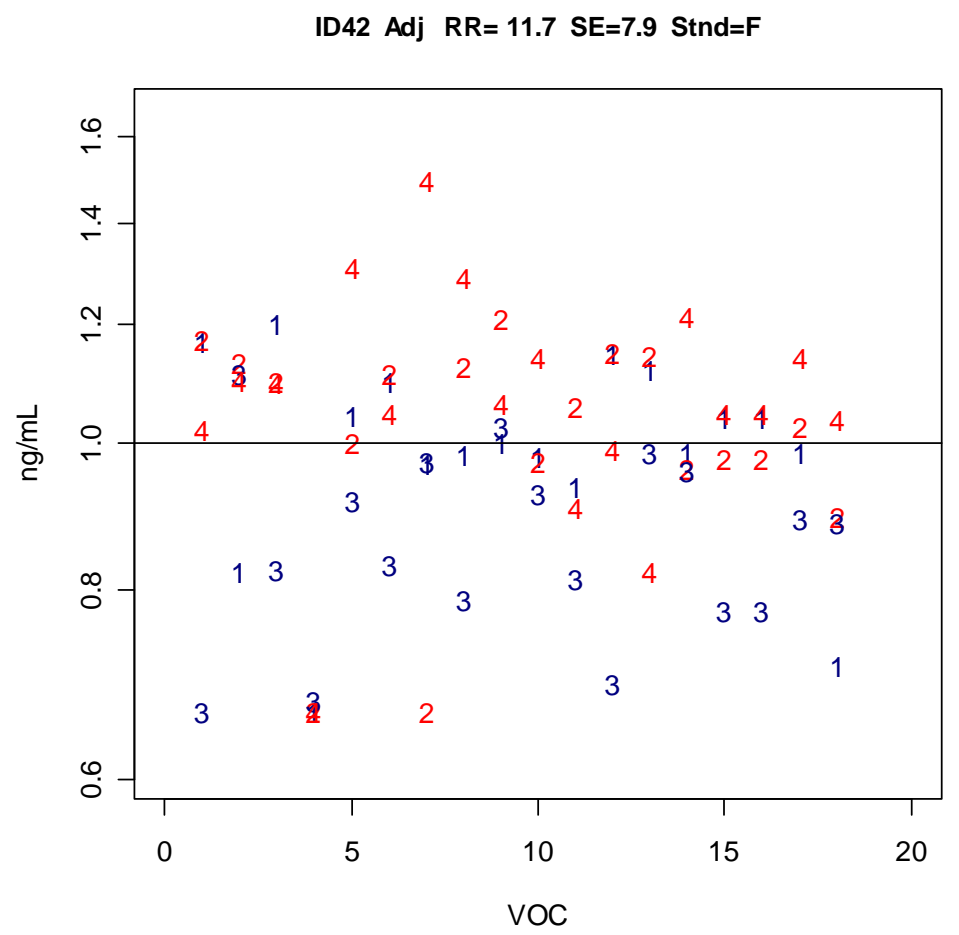

Figure E10. Statistical results for ID42 


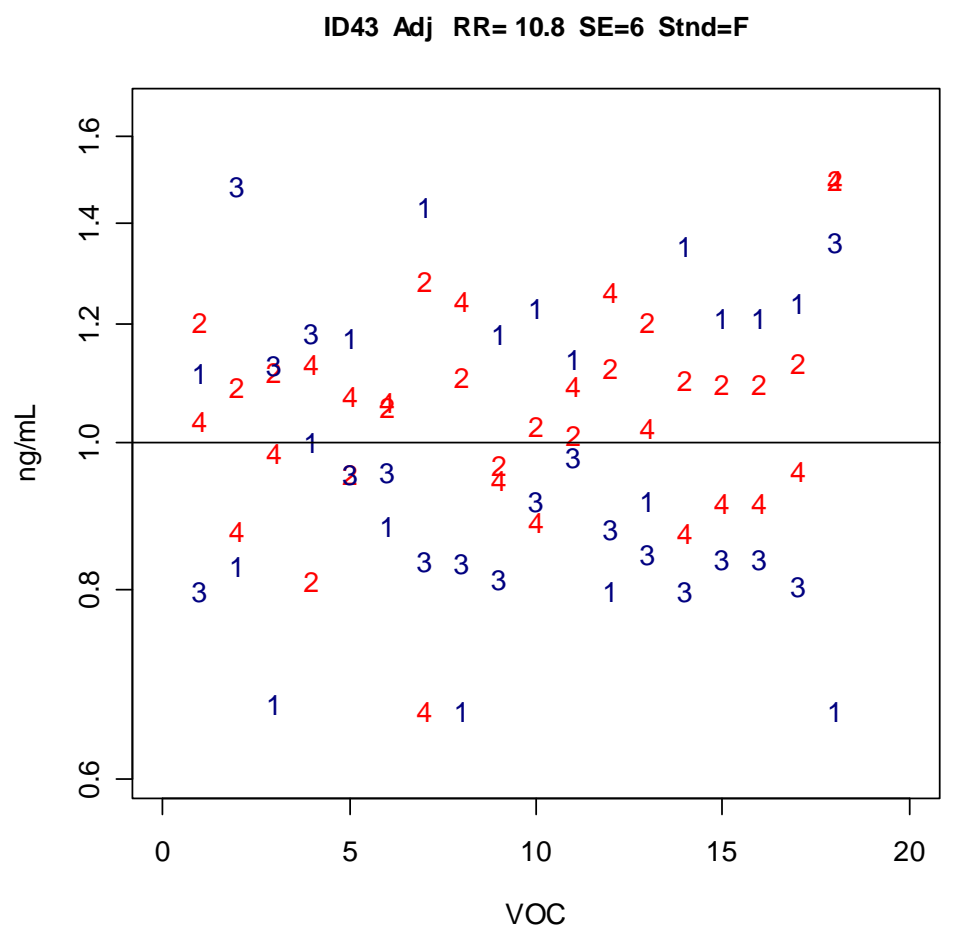

Figure E11. Statistical results for ID43

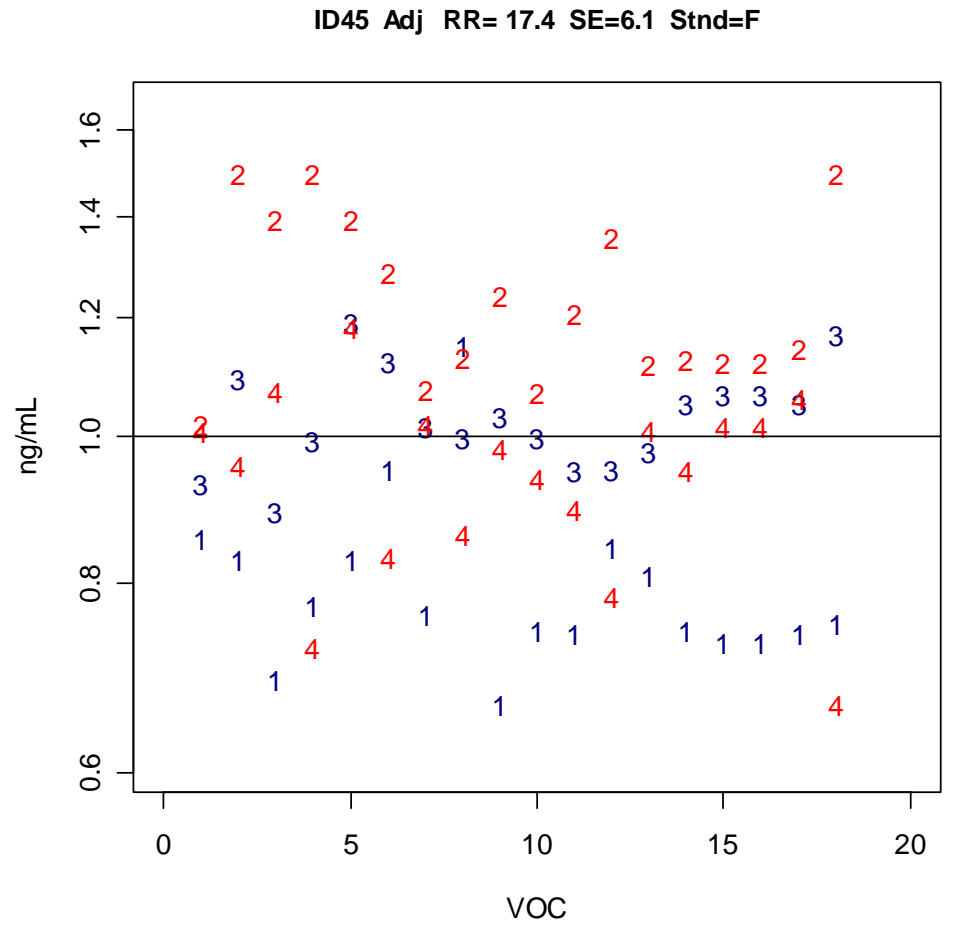

Figure E12. Statistical results for ID45 


\section{Appendix F. Statistical Results Based on Groups of Chemically Related Vocs}

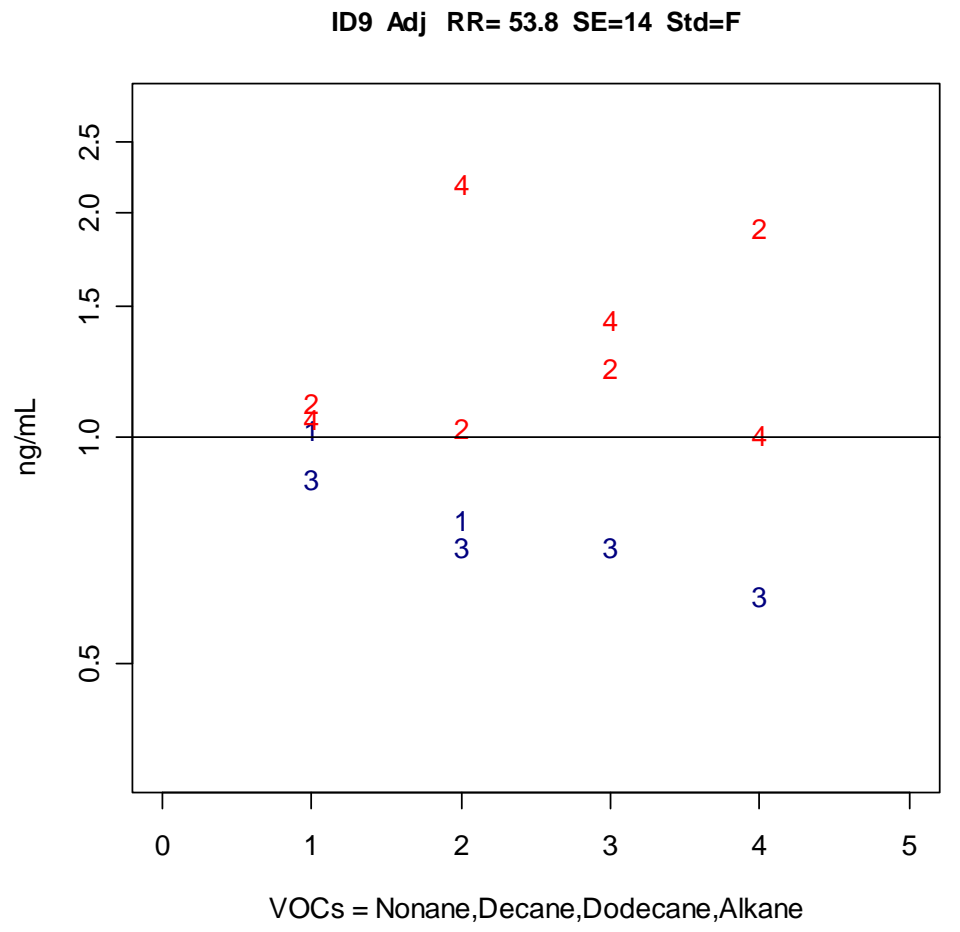

Figure F1: ID9 VOC group 2

ID9 Adj RR= 39.5 SE=4.4 Std=F

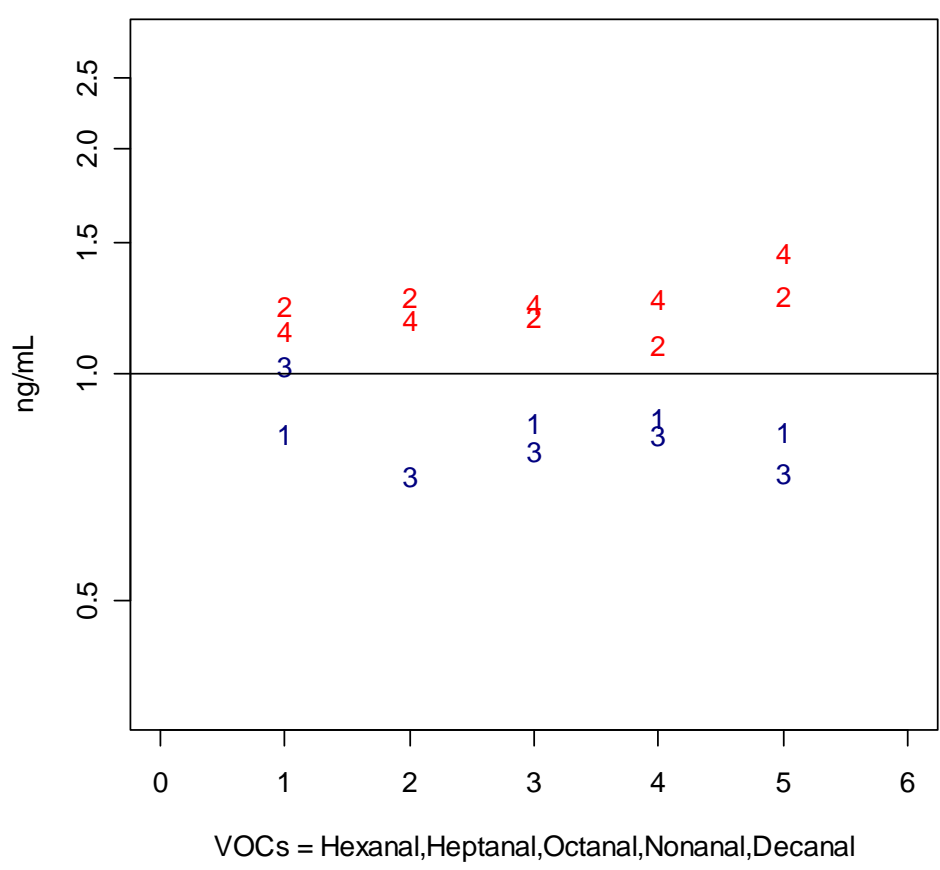

Figure F2. ID9 VOC group 3 
ID9 Adj $R R=22.1$ SE=4.2 Std=F

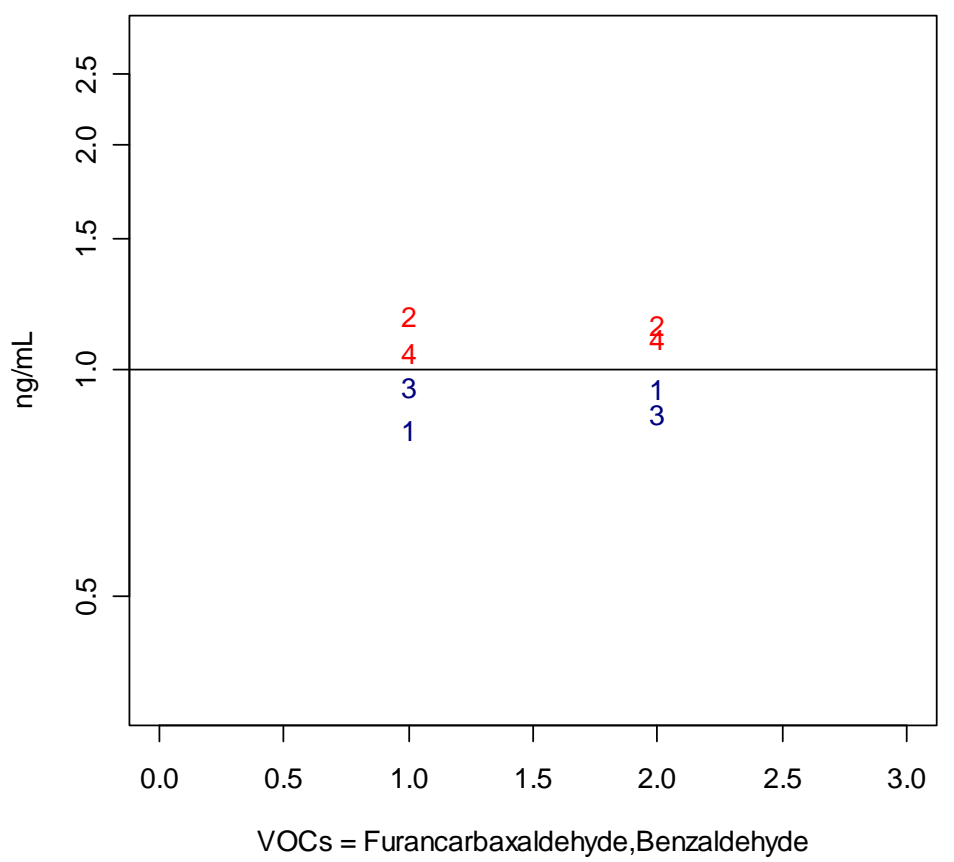

Figure F3. ID9 VOC group 4

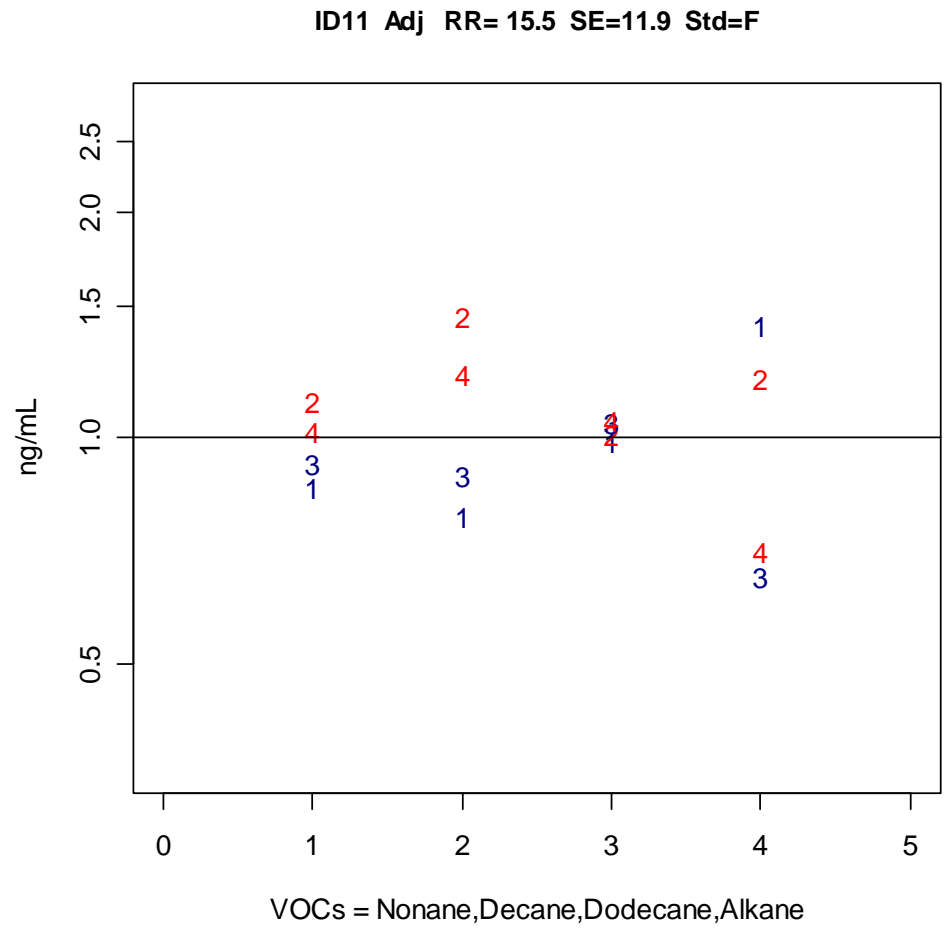

Figure F4. ID11 VOC group 2 
ID11 Adj RR=15 SE=8.3 Std=F

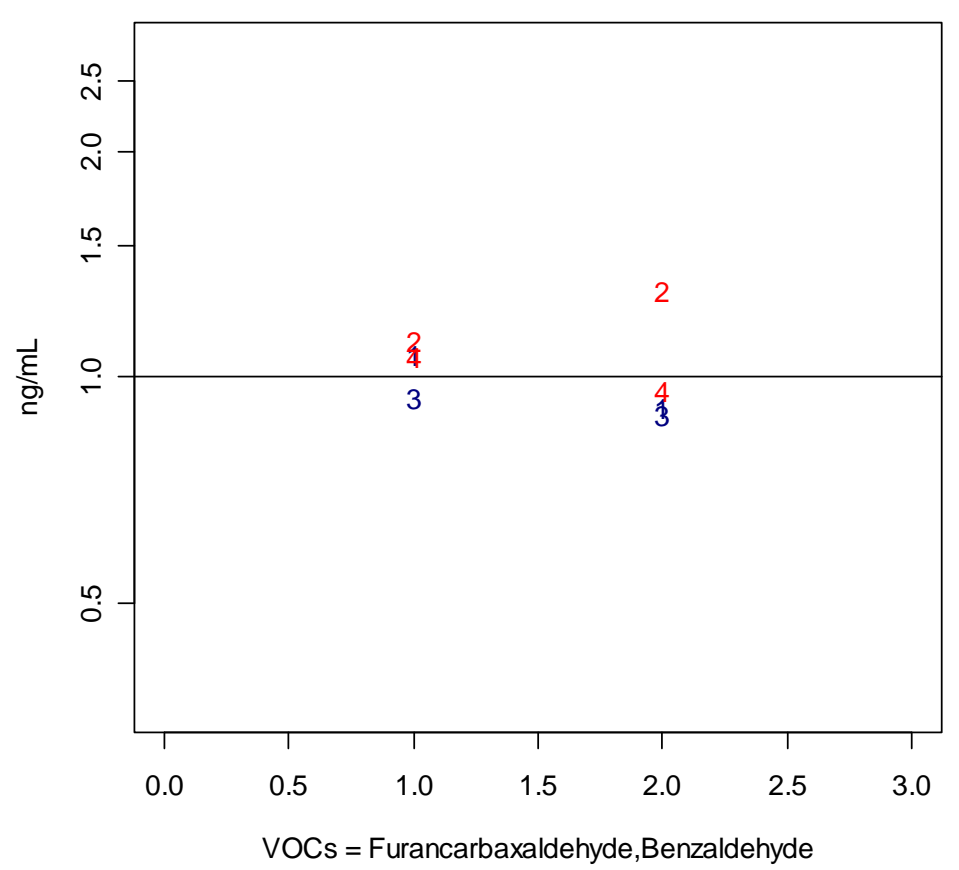

Figure F5. ID11 VOC group 4

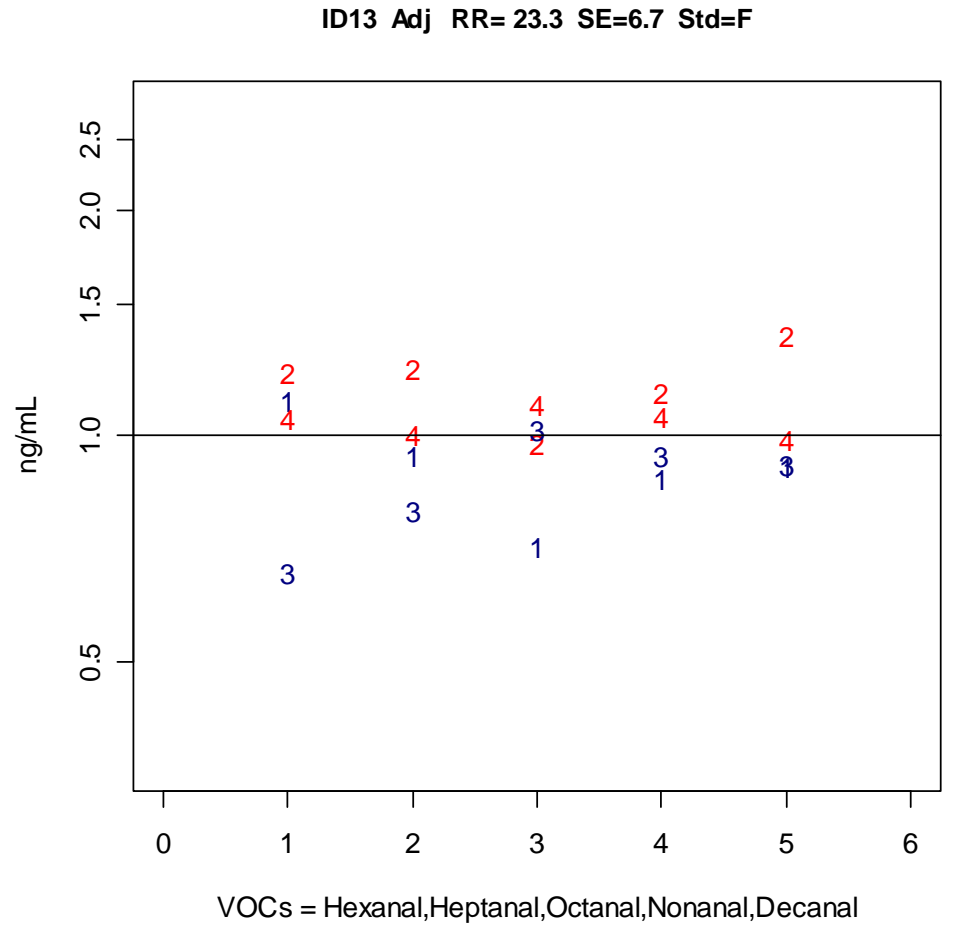

Figure. F6. ID13 VOC group 3 


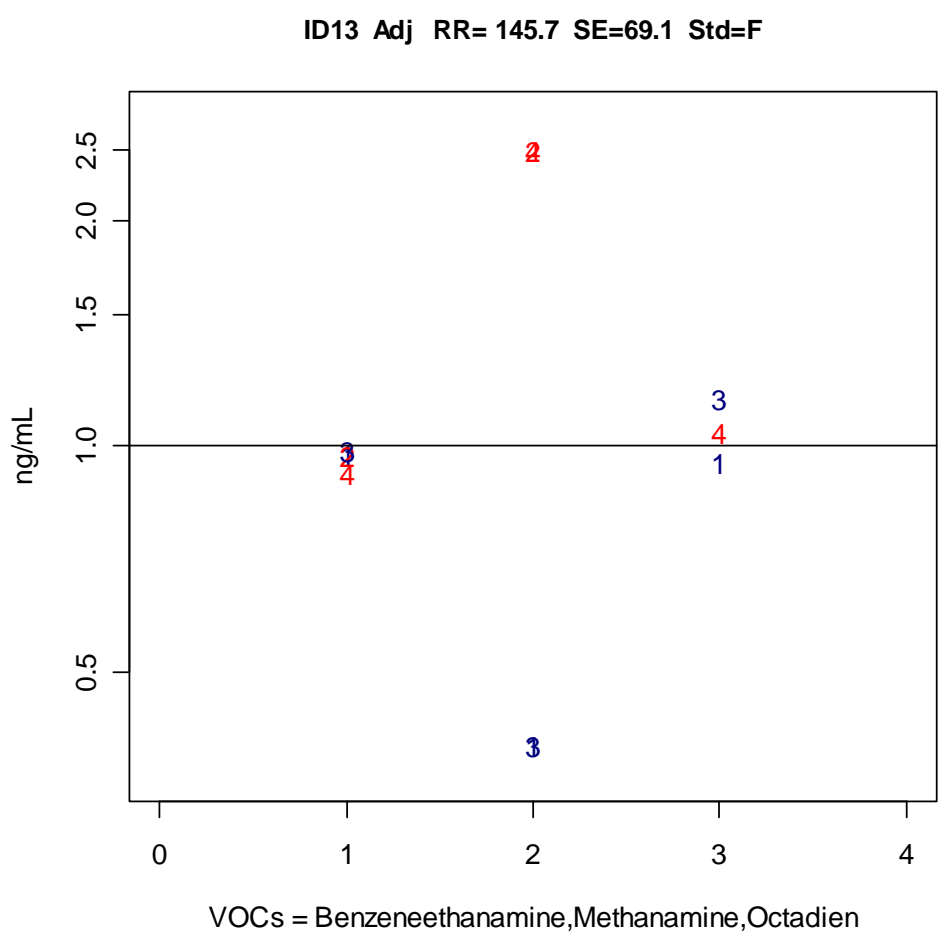

Figure F7. ID13 VOC group 5

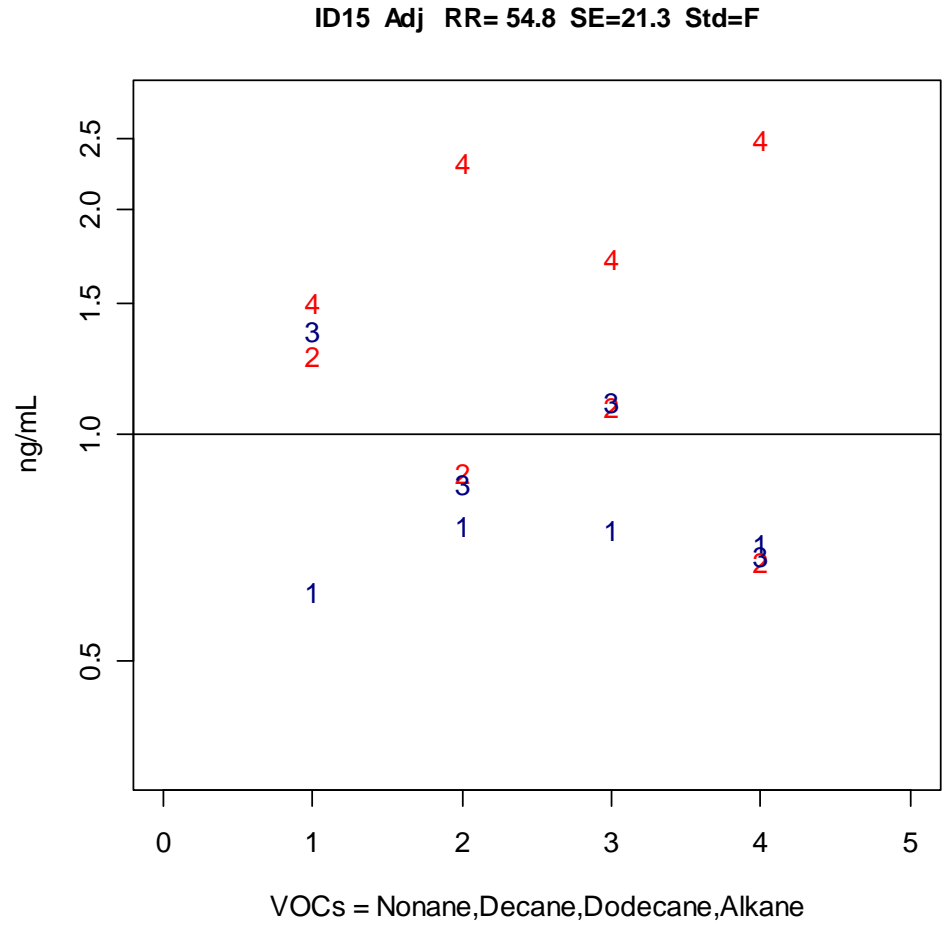

Figure F8. ID15 VOC group 2 
ID15 Adj RR= 123.9 SE=54.6 Std=F

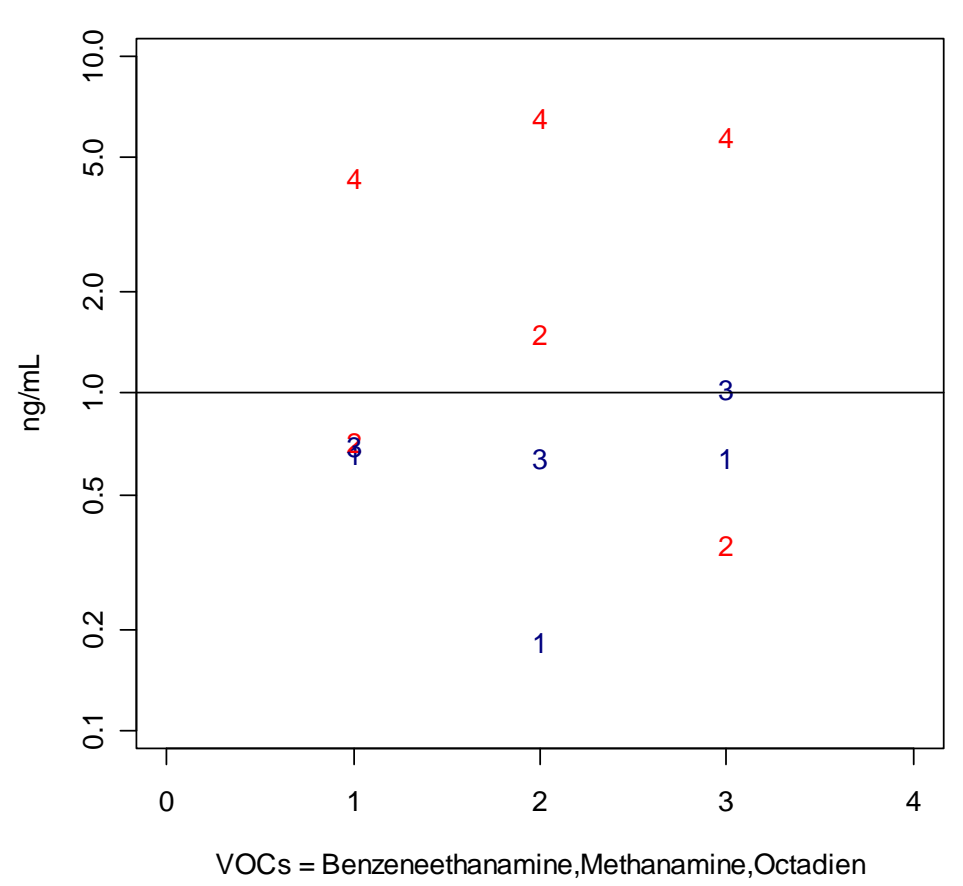

Figure F9. ID15 VOC group 5

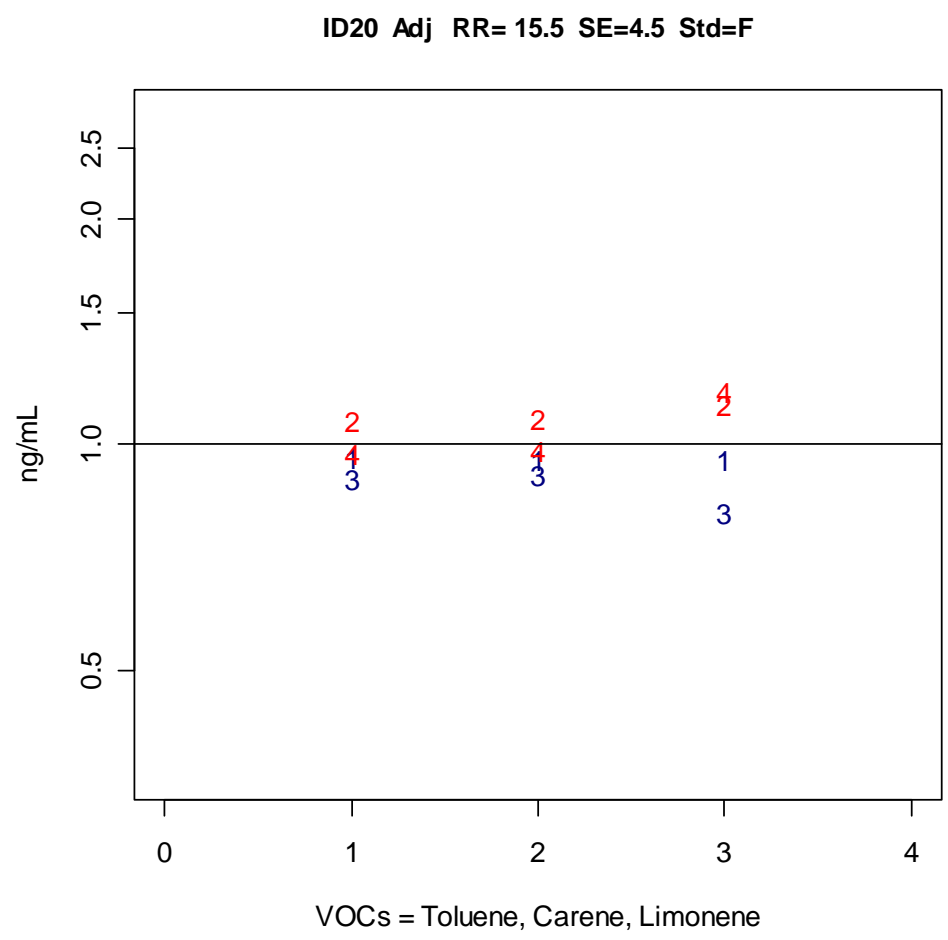

Figure F10. ID20 VOC group 1 
ID20 Adj RR= 26.3 SE=5.4 Std=F

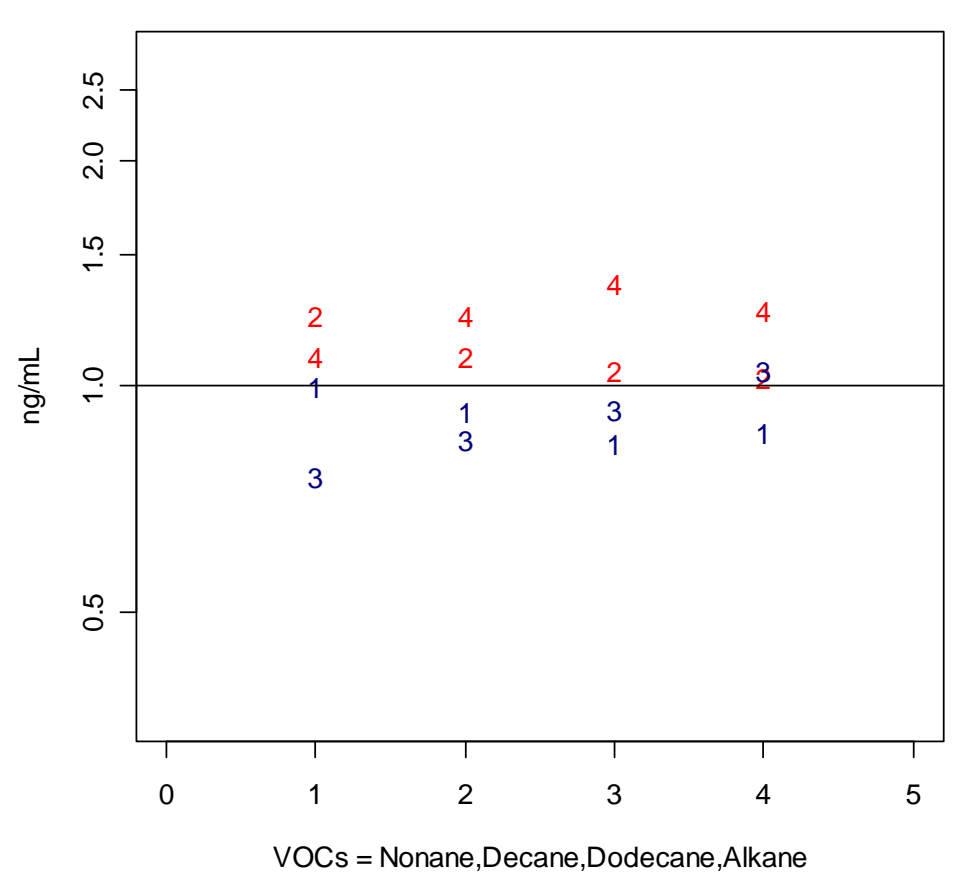

Figure F11. ID20 VOC group 2

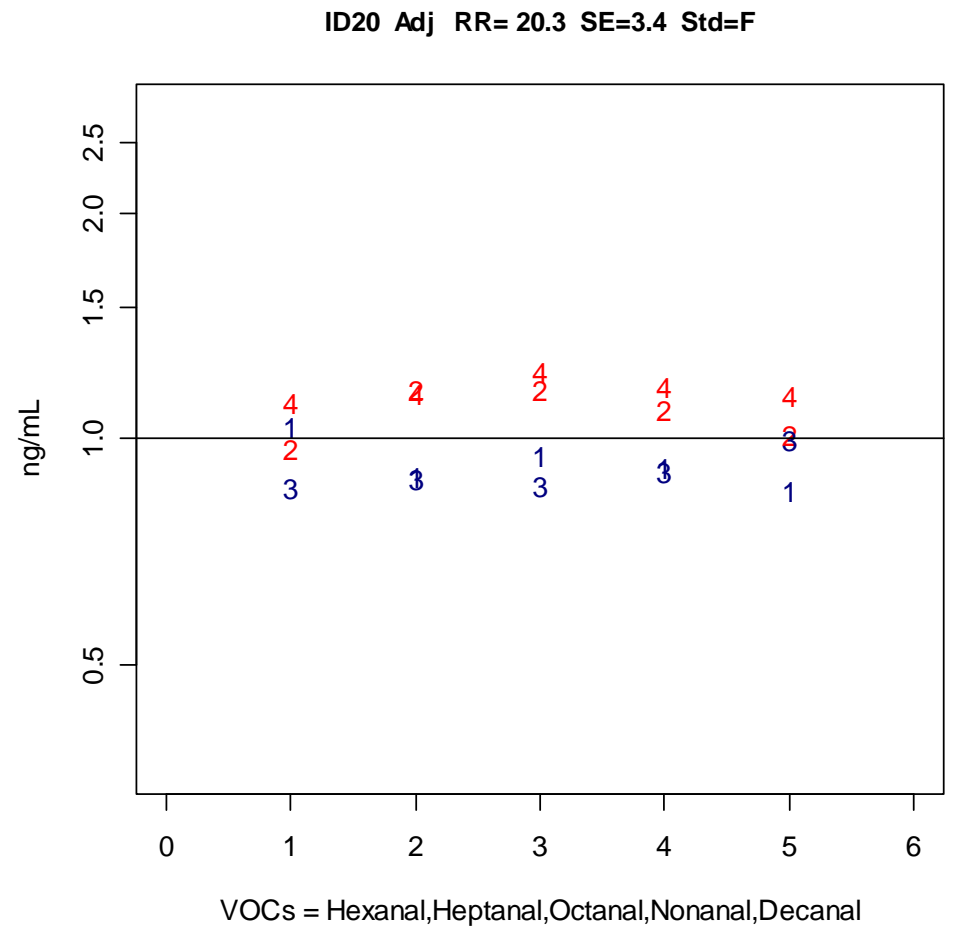

Figure F12. ID20 VOC group 3 


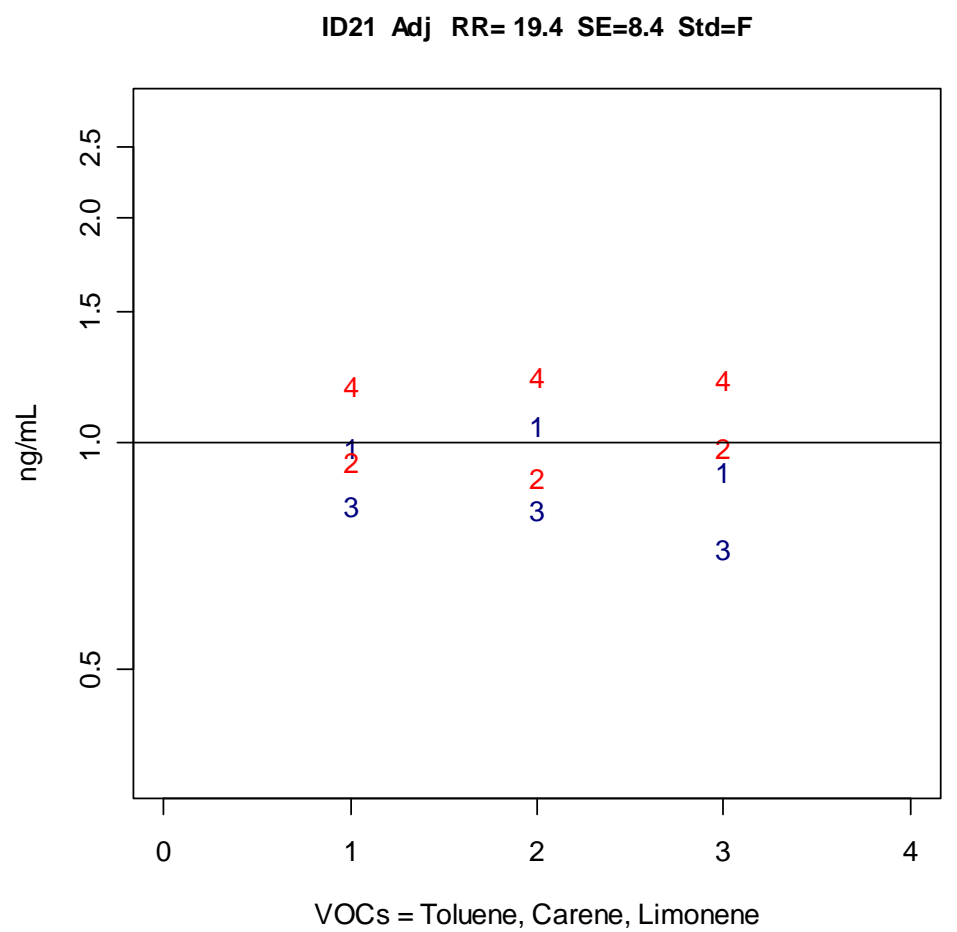

Figure F13. ID21 VOC group 1

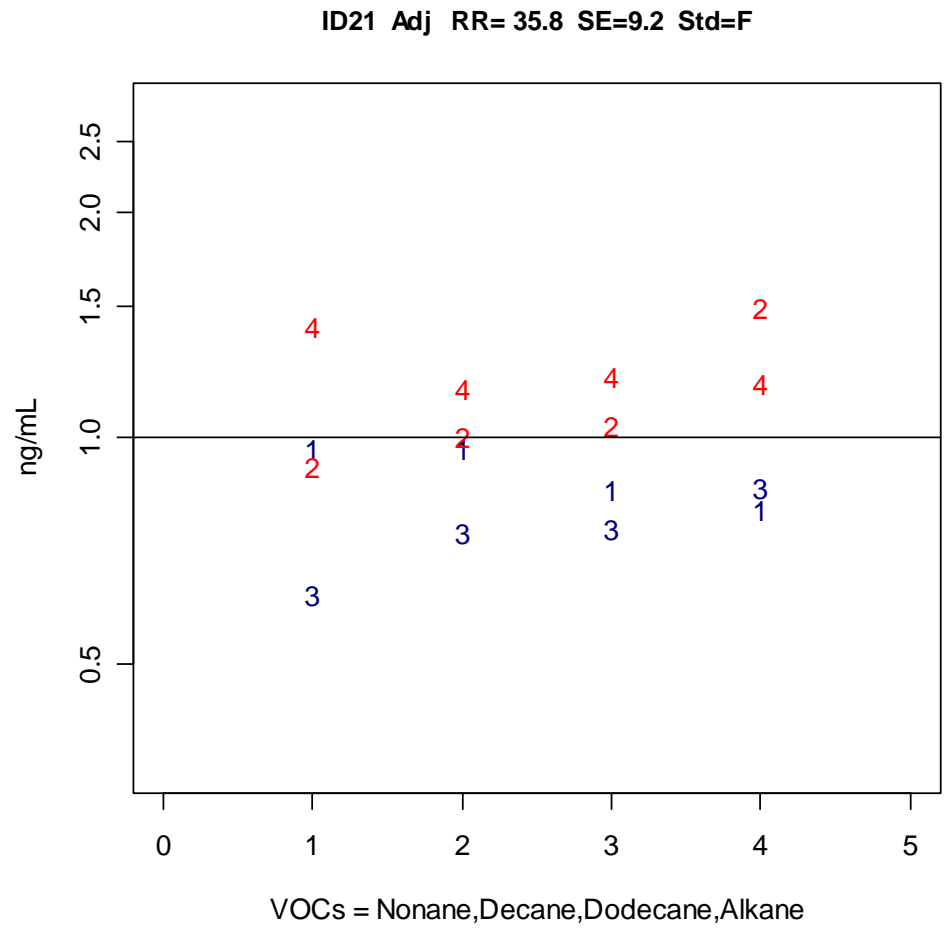

Figure F14. ID21 VOC group 2 
ID21 Adj RR= 27.9 SE=7.1 Std=F

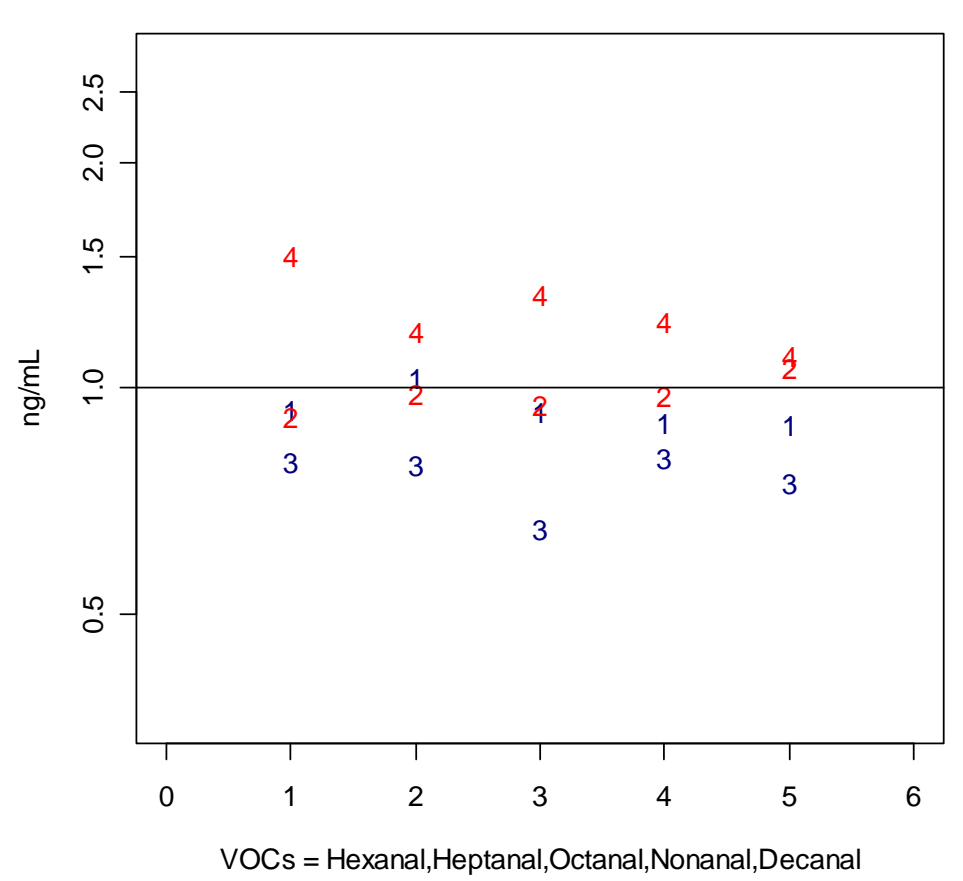

Figure F15. ID21 VOC group 3

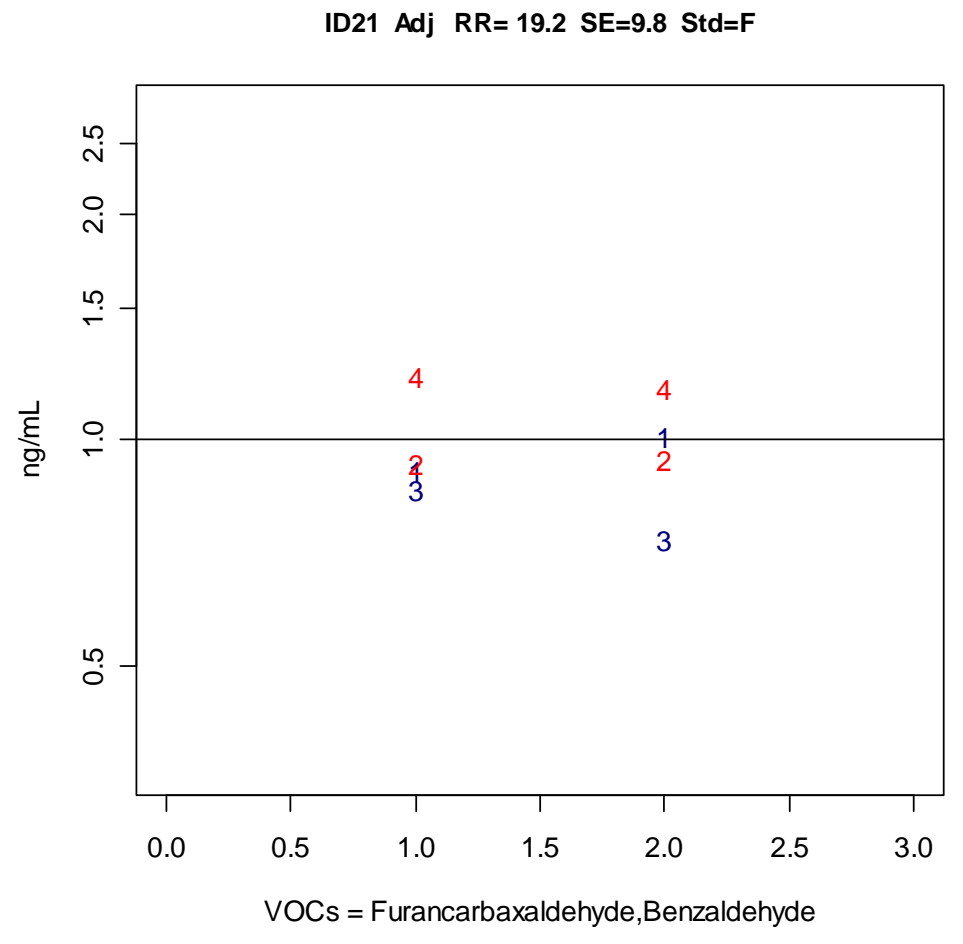

Figure F16. ID21 VOC group 4 


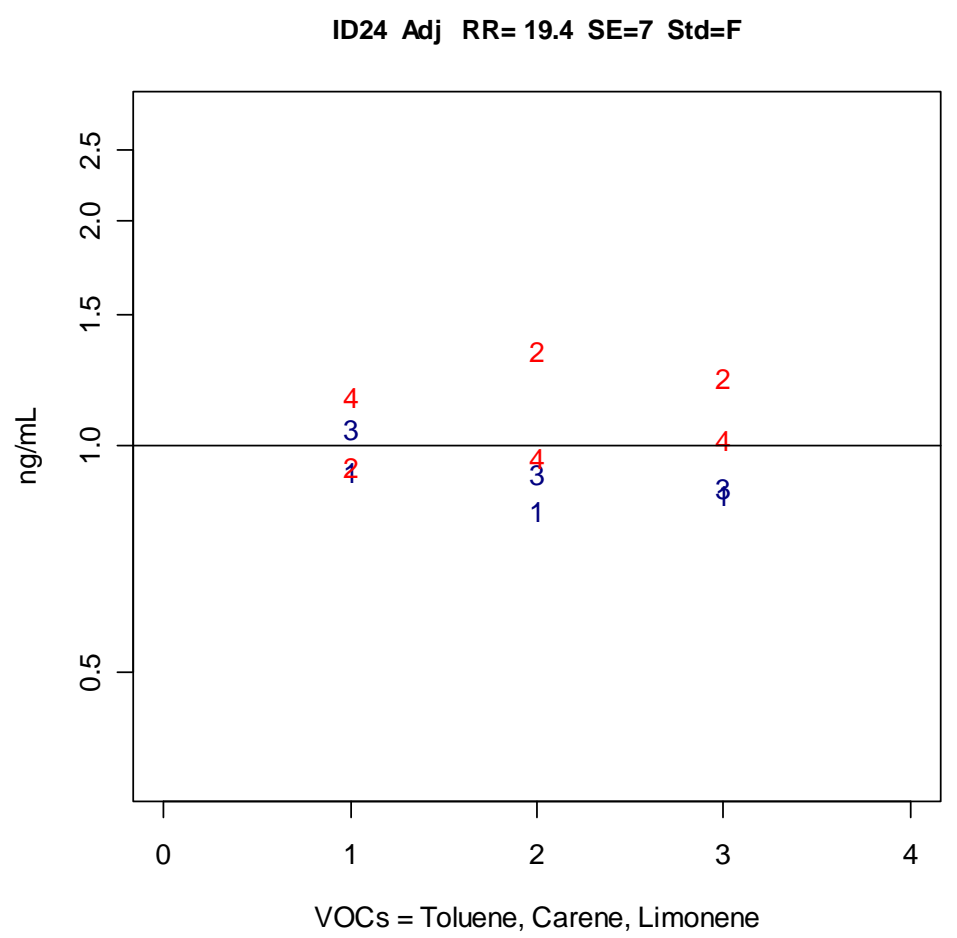

Figure F17. ID24 VOC group 1

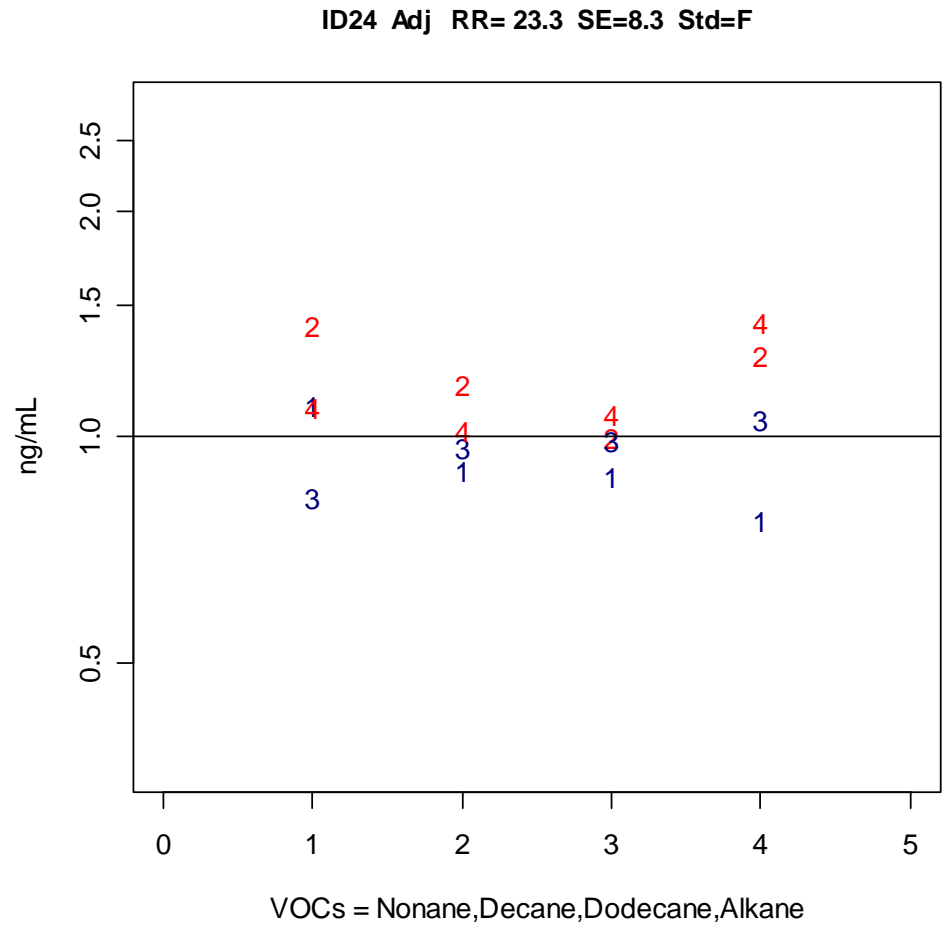

Figure F18. ID24 VOC Group 2 


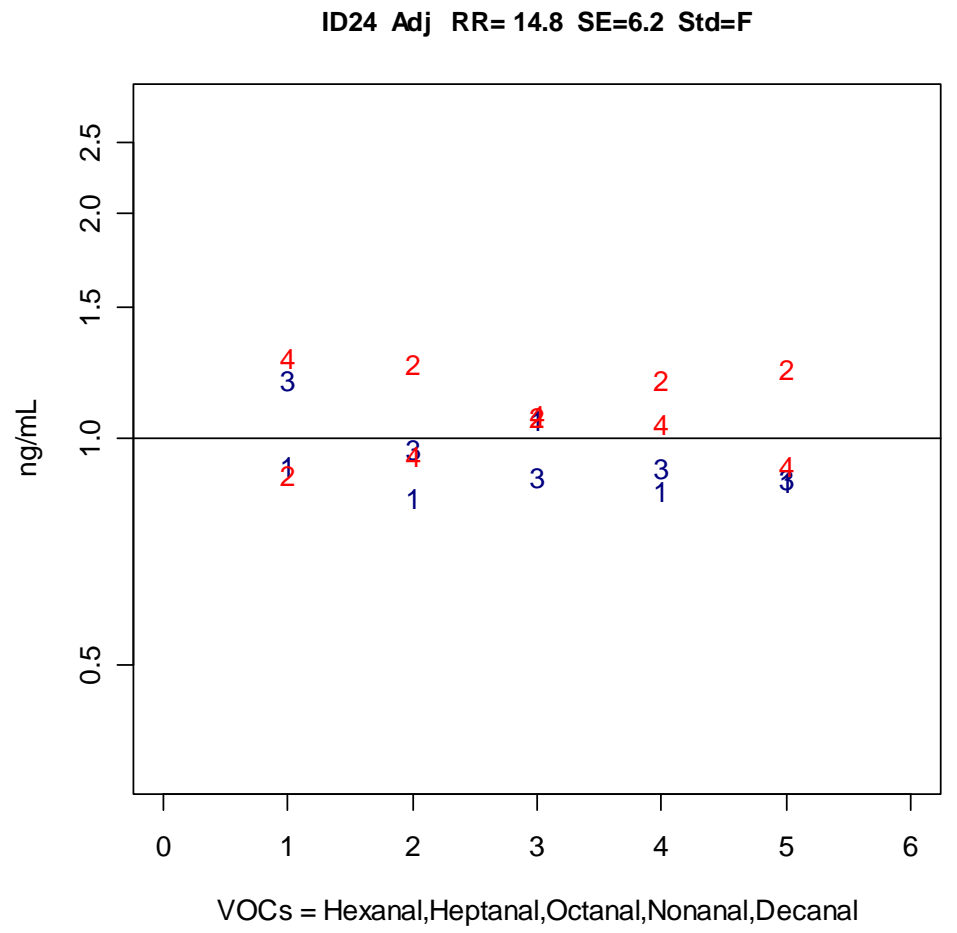

Figure F19. ID24 VOC group 3

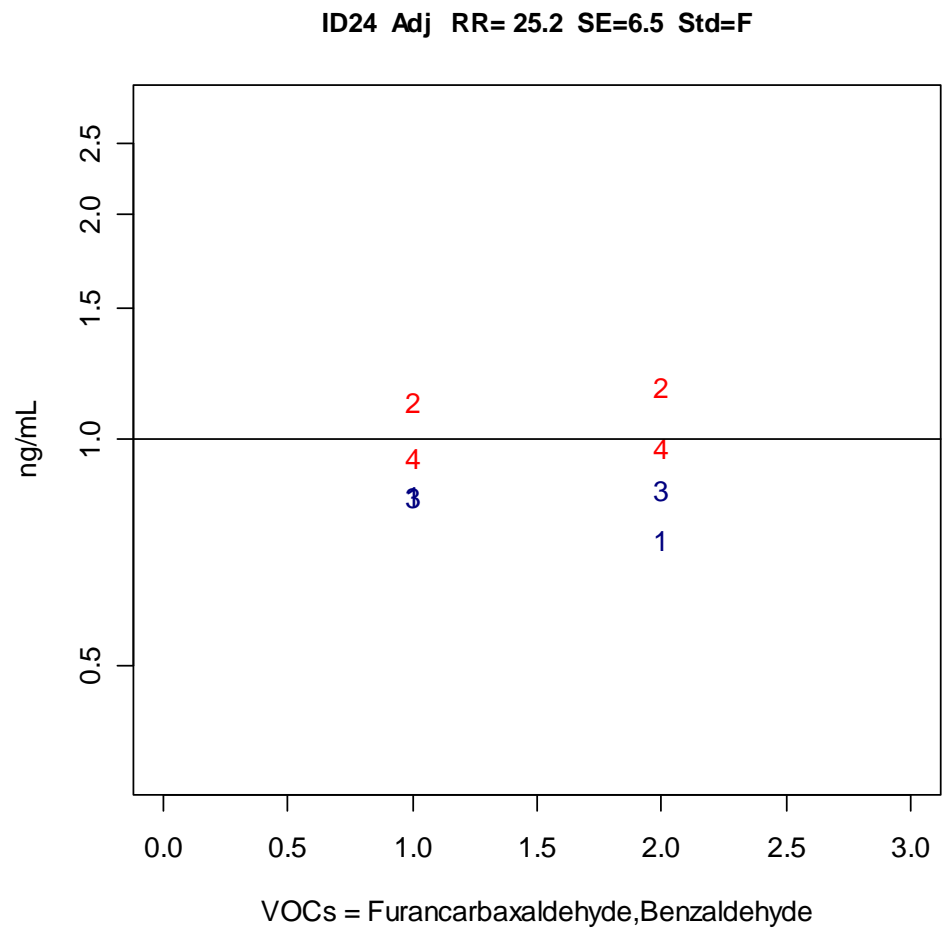

Figure F20. ID24 VOC group 4 


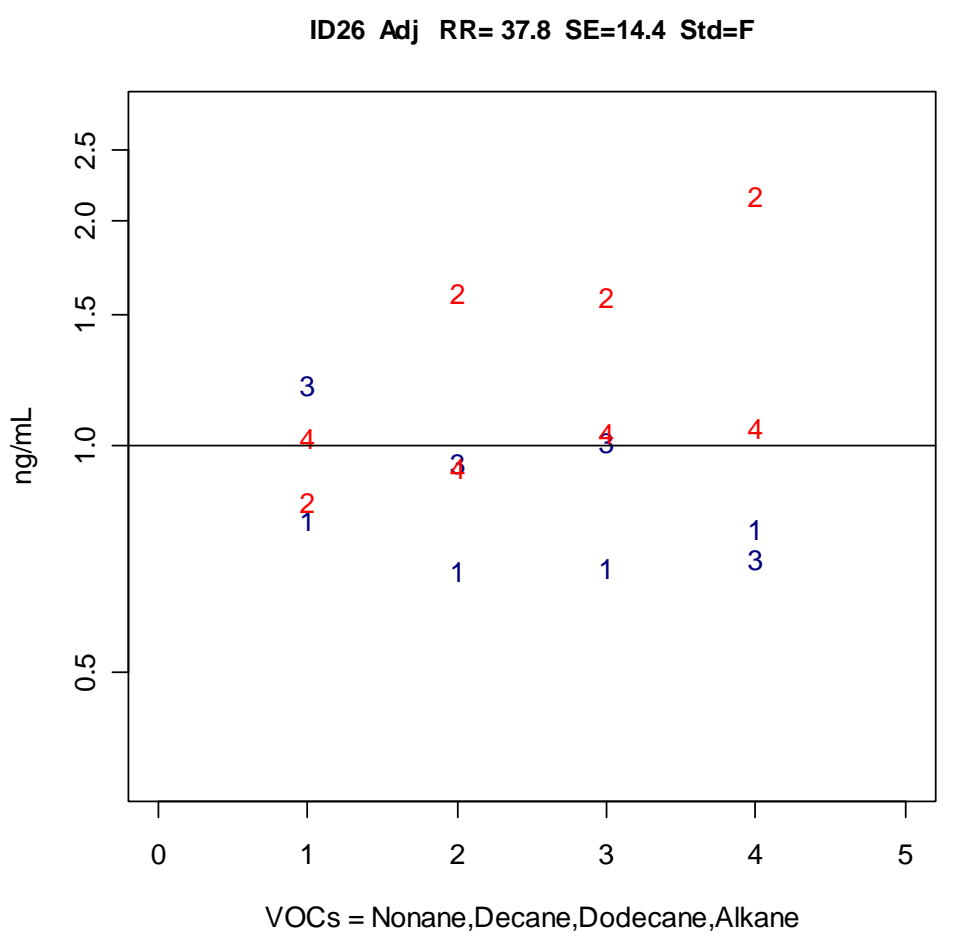

Figure F21. ID26 VOC group 2

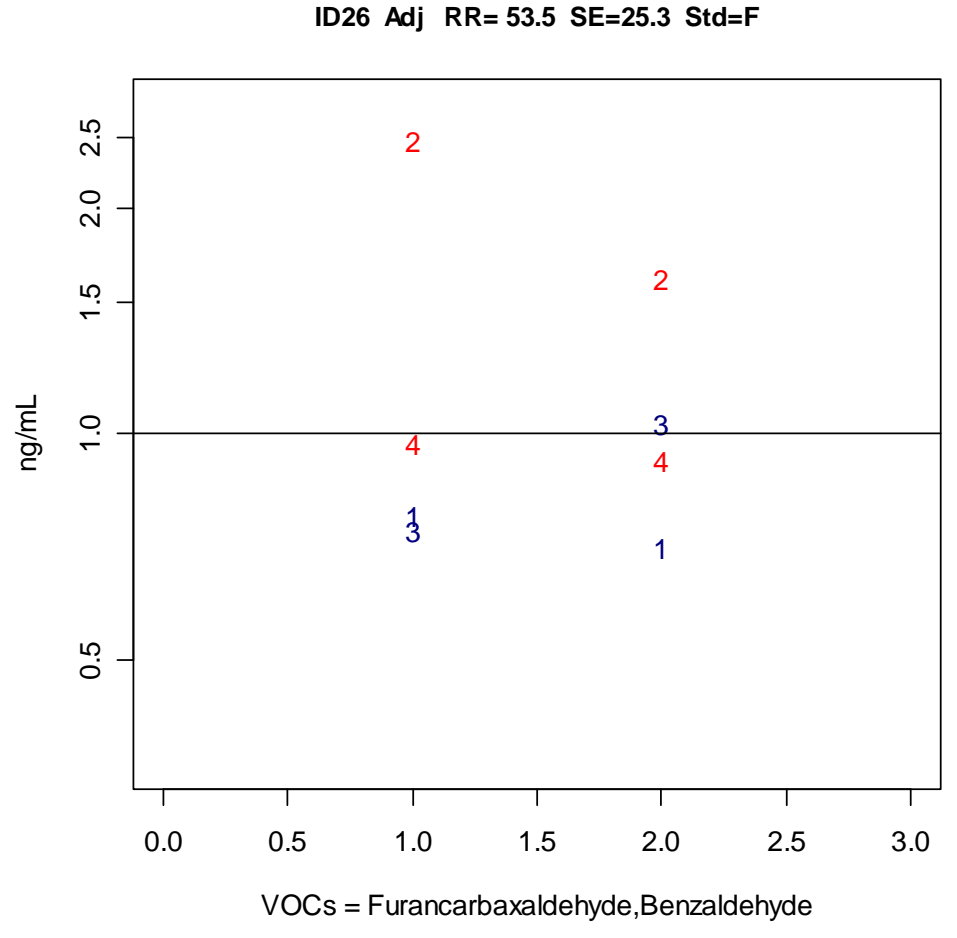

Figure F22. ID26 VOC group 4 


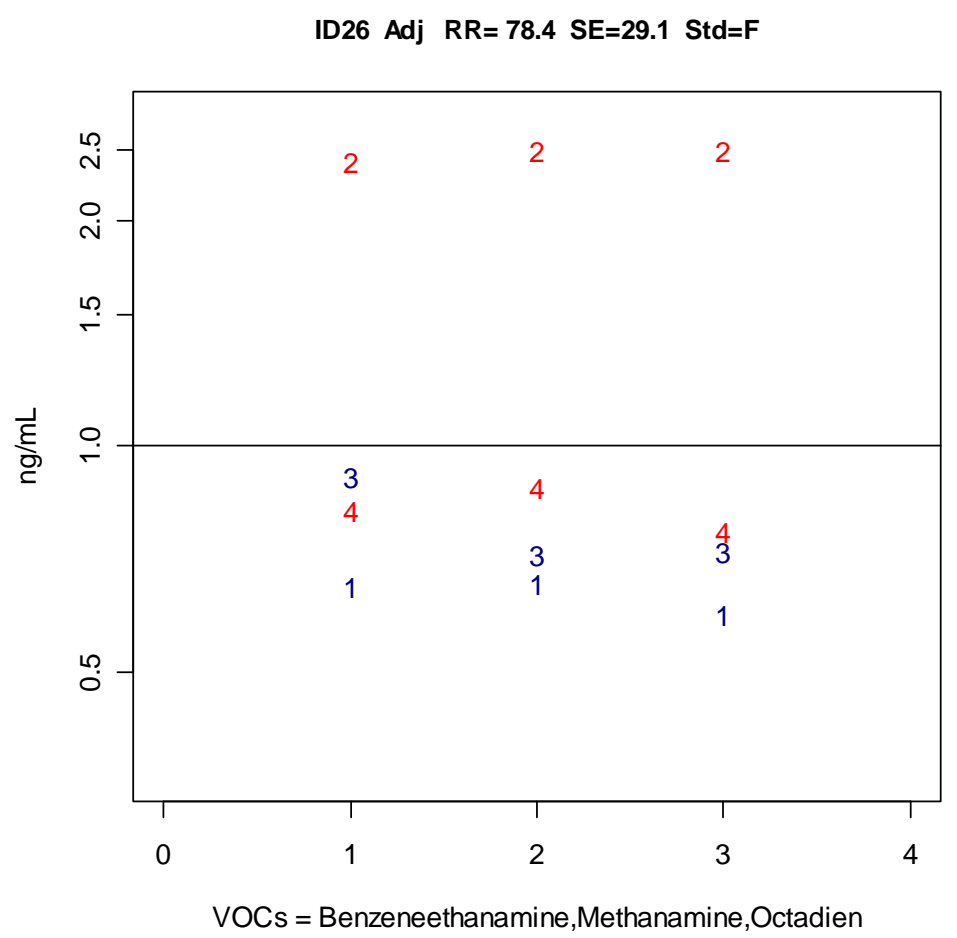

Figure F23. ID26 VOC group 5

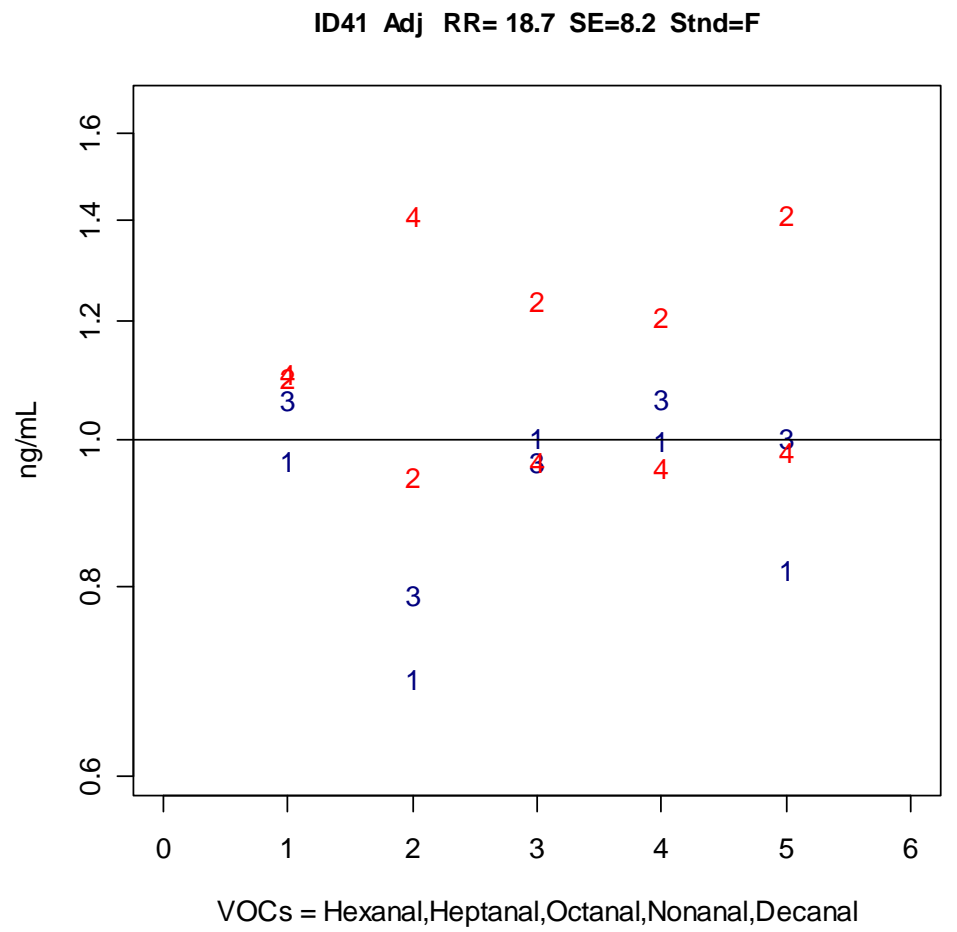

Figure F24. ID41 VOC group 3 


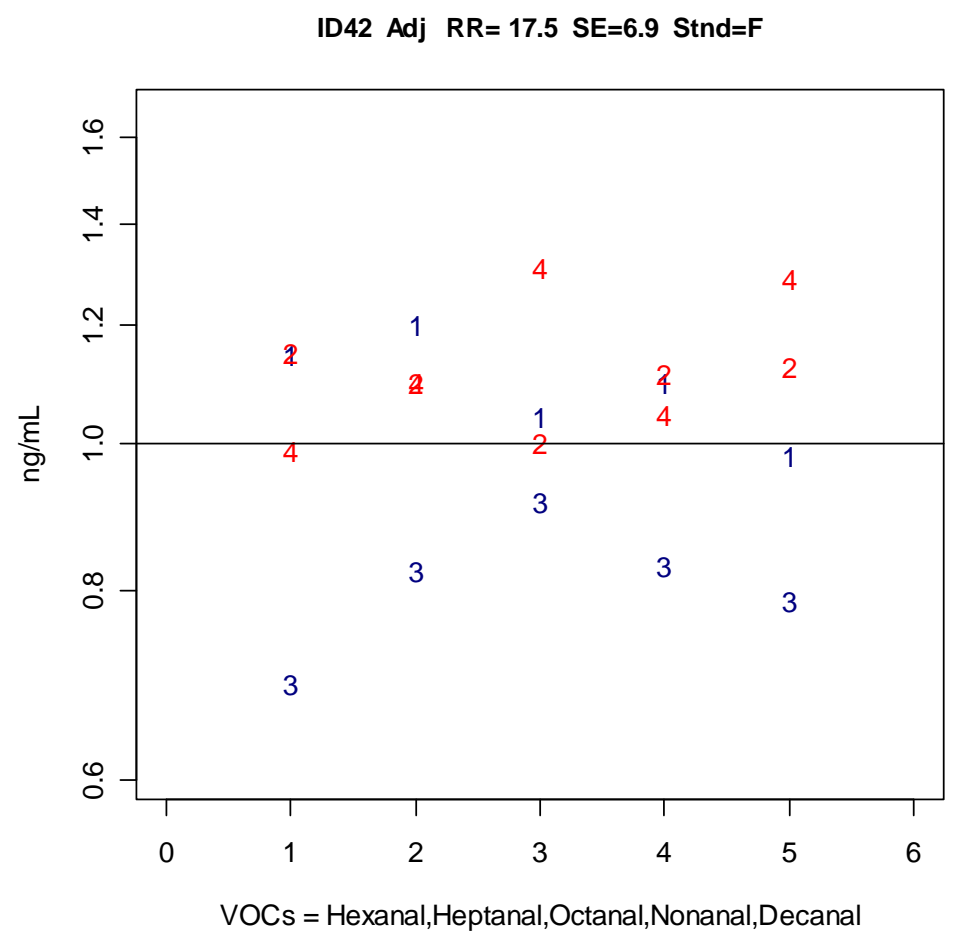

Figure F25: ID42 VOC group 3

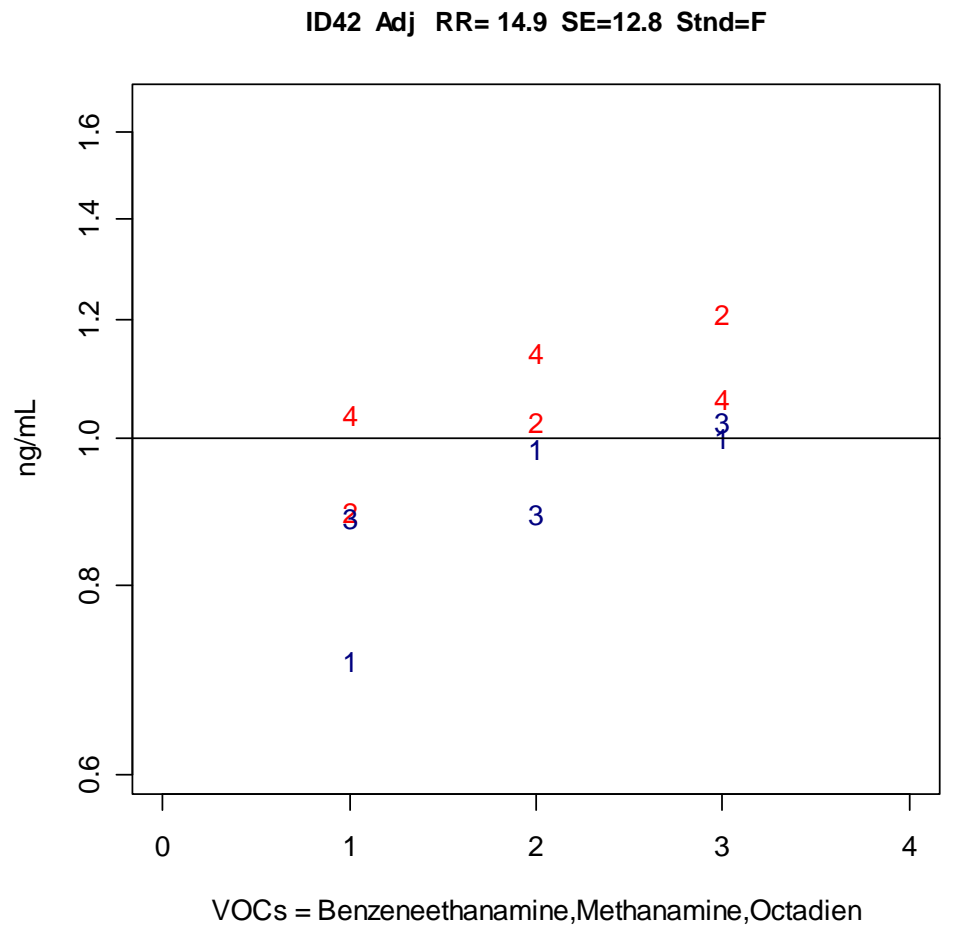

Figure F26. ID42 VOC group 5 


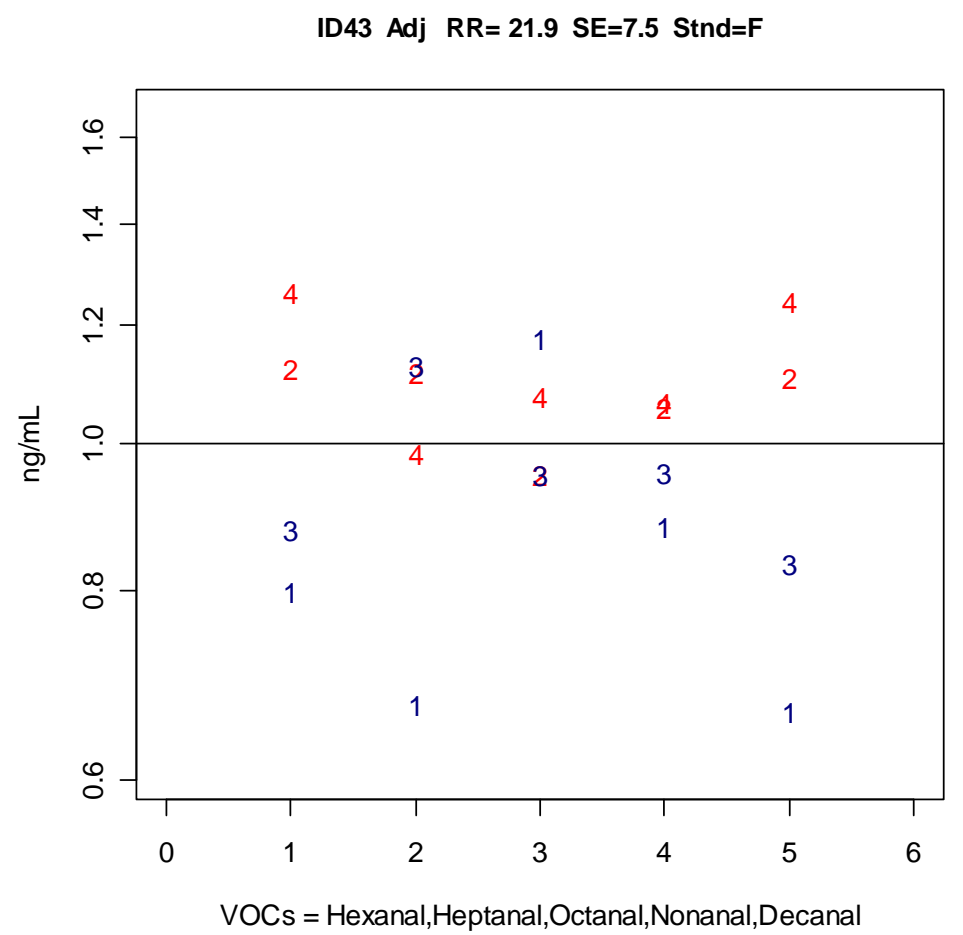

Figure F27: ID43 VOC group 3

ID45 Adj RR= 15.4 SE=7.1 Stnd=F

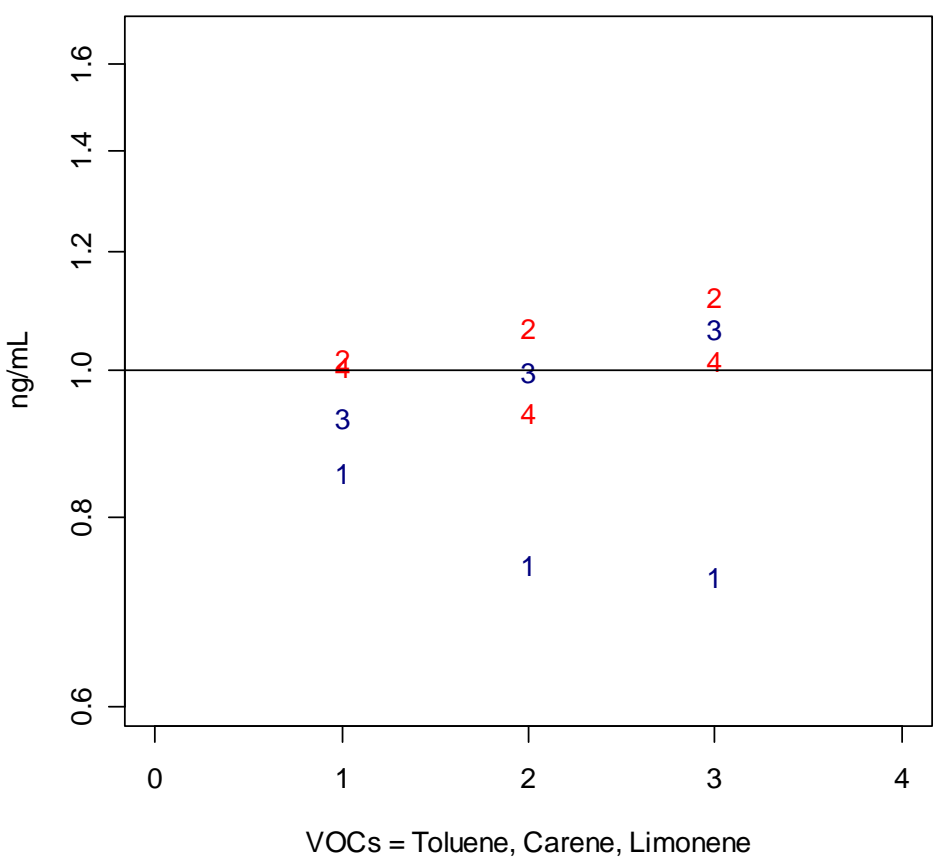

Figure F28: ID45 VOC group 5 